Universidade de São Paulo

Instituto de Física

\title{
Espalhamento e absorção de campos bosônicos por buracos negros estáticos e análogos
}

\author{
Ednilton Santos de Oliveira
}

Orientador: Prof. Dr. Luís Carlos Bassalo Crispino

Tese de doutorado apresentada ao Instituto de Física para a obtenção

do título de Doutor em Ciências

Banca examinadora:

Prof. Dr. Luís Carlos Bassalo Crispino (UFPA)

Prof. Dr. Victor de Oliveira Rivelles (IFUSP)

Prof. Dr. Paulo Teotônio Sobrinho (IFUSP)

Prof. Dr. George Emanuel Avraam Matsas (IFT/UNESP)

Prof. Dr. Patricio Anibal Letelier Sotomayor (UNICAMP)

São Paulo

2009 


\section{FICHA CATALOGRÁFICA}

\section{Preparada pelo Serviço de Biblioteca e Informação}

do Instituto de Física da Universidade de São Paulo

Oliveira, Ednilton Santos de

Espalhamento e absorção de campos bosônicos por buracos negros estáticos e análogos - São Paulo, 2009.

Tese (Doutorado) - Universidade de São Paulo. Instituto de Física. Universidade Federal do Pará. Faculdade de Física. Departamento Interunidades

Orientador: Prof. Dr. Luís Carlos Bassalo Crispino

Área de Concentração: Teoria Quântica de Campos em Espaços Curvos

Unitermos: 1. Relatividade (Física); 2. Teoria de campos e ondas; 3. Física matemática; 4. Mecânica quântica; 5. Teoria quântica relativística. 


\section{Agradecimentos}

A Deus.

Ao Prof. Dr. Luís C. B. Crispino pela grande dedicação e competência com que me orienta desde a minha Iniciação Científica. Também, pelos bons conselhos e por ter me repassado muito da sua experiência.

Aos Profs. Drs. Atsushi Higuchi, George E. A. Matsas e Sam R. Dolan cujas colaborações me proporcionaram bastante conhecimento e resultados ricos já obtidos e ainda por serem alcançados.

À Comissão de Pós-Graduação do Instituto de Física da Universidade de São Paulo, pela oportunidade concedida para que eu pudesse continuar trabalhando com o professor Luís C. B. Crispino.

À CAPES e ao CNPq, pelo suporte financeiro desde a minha Iniciação Científica.

À Universidade Federal do Pará na qual desenvolvi a graduação e o mestrado.

Aos meus pais, Nilton e Edinilza, à minha irmã Merynilza, por todo apoio, pelos momentos de descontração e por tornarem a minha vida mais feliz.

Ao meu primo Josiel e ao meu amigo Paulo, pela grande amizade.

Aos componentes do grupo de Teoria Quântica de Campos em Espaços Curvos da UFPA, com os quais tenho aprendido bastante.

Ao colega do Instituto de Física da USP e conterrâneo, Rodrigo Gester, que tem sido um grande amigo desde o início deste doutorado.

À Escola Edinair Rocha onde estudei o ensino básico.

À Escola Municipal de Ensino Fundamental Cândida Santos de Souza onde realizei meu ensino fundamental.

Ao Colégio Estadual Pedro Amazonas Pedroso, onde iniciei e concluí meu ensino médio.

A todos que direta ou indiretamente contribuíram para que este projeto de doutorado tivesse seus objetivos cumpridos. 


\section{Resumo}

Nesta tese apresentamos a análise da absorção e do espalhamento de partículas não massivas de spins 0 e 1 por buracos negros de Schwarzschild, assim como a absorção e espalhamento por análogos acústicos destes buracos negros. Apresentamos também a análise da absorção e do espalhamento do campo escalar não massivo por buracos negros de Reissner-Nordström. A presente pesquisa se baseia no método de decomposição em ondas parciais. Devido a estes espaços-tempos serem estáticos e esfericamente simétricos, as partes temporal e angular das soluções das equações de campo se reduzem a funções conhecidas. O mesmo não acontece com a parte radial, o que nos leva a abordar o problema, principalmente, por meio de métodos numéricos. Cálculos analíticos também são realizados, geralmente para podermos verificar a precisão dos resultados numéricos. Os principais resultados analíticos mostrados aqui são a seção de choque de absorção em baixas e altas frequências e a seção de choque diferencial de espalhamento para ângulos próximos a $180^{\circ}$ (efeito glória). Mostramos, de forma analítica, que as principais características das seções de choque de absorção e diferencial de espalhamento estão diretamente relacionadas à existência da órbita instável de partículas não massivas. Os nossos resultados numéricos estão em excelente concordância com os resultados obtidos por meio de cálculos analíticos. Com relação ao espaço-tempo de Reissner-Nordström, mostramos qual o comportamento das seções de choque com a variação da intensidade de carga do buraco negro. No caso de buracos negros descarregados, fazemos comparações das seções de choque obtidas para partículas de diferentes spins.

Palavras Chave: Buracos negros estáticos, buracos acústicos canônicos, campo eletromagnético, campo escalar não massivo, seções de choque.

Áreas de Conhecimento: 1.05.00.00-6, 1.05.01.02-9, 1.05.01.03-7 


\begin{abstract}
We present an analysis of the absorption and scattering of massless particles of spin 0 and 1 by Schwarzschild black holes, and the absorption and scattering by analogues of these black holes. We present also the analysis of the massless scalar field absorption and scattering by Reissner-Nordström black holes. This research is based on the partial wave methods. Since these spacetimes are static and spherically symmetric, the time and angular dependence of the field equation solutions can be written in terms of well known functions. The same does not happen with the radial part of the field equation solutions, so that we apply numerical methods to solve the absorption and scattering problem. Analytical computations are also performed and we use them to verify the precision of our numerical computations. The main analytical results obtained here are the low- and high-frequency absorption cross sections and the differential scattering cross section for angles near $180^{\circ}$ (the glory effect). We use our analytical results to show that the main cross sections properties are related to the existence of an unstable orbit for massless particles. We compare our numerical results with semiclassical approximations from a geodesic analysis, and find excellent agreement. In the Reissner-Nordström spacetime case, we show how the cross sections behave as we vary the black hole charge. For uncharged black holes, we compare cross sections for particles with different spins.
\end{abstract}

Keywords: Static black holes, canonical acoustic hole, electromagnetic field, massless scalar field, cross section. 


\section{Sumário}

1 Introdução 11

2 Os espaços-tempos

2.1 Buracos negros de Reissner-Nordström . . . . . . . . . . . . . . . 15

2.2 Buracos acústicos canônicos . . . . . . . . . . . . . 21

3 Os campos 25

3.1 Campo escalar não massivo em Reissner-Nordström . . . . . . . . . . . . . . . 25

3.2 Perturbações em buracos acústicos canônicos . . . . . . . . . . . . . . 29

3.3 O campo eletromagnético no espaço-tempo de Schwarzschild . . . . . . . . . . 31

3.3.1 Equações de Maxwell na geometria de Schwarzschild . . . . . . . . . 32

3.3.2 Soluções no gauge de Coulomb esférico . . . . . . . . . . . . . . . 33

3.3.3 Soluções no gauge de Feynman modificado . . . . . . . . . . . . . . 38

3.3.4 Soluções assintóticas . . . . . . . . . . . . . . . . 39

4 Seção de choque 41

4.1 Definições . . . . . . . . . . . . . . . . . . . . . . 41

4.2 Seção de choque de absorção em baixas frequências . . . . . . . . . . . . . . 43

4.2.1 Caso escalar em Schwarzschild . . . . . . . . . . . . . . . . . 43

4.2.2 Caso escalar em Reissner-Nordström extremo . . . . . . . . . . . . . 46

4.2.3 Buracos acústicos canônicos . . . . . . . . . . . . 47

4.2.4 Caso eletromagnético em Schwarzschild . . . . . . . . . . . . . . . 48

4.3 Espalhamento com pequenos ângulos . . . . . . . . . . . . . . 50

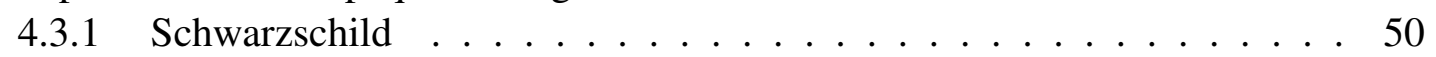

4.3.2 Reissner-Nordström . . . . . . . . . . . . . . . . 52 52

4.3.3 Buraco acústico canônico . . . . . . . . . . . . 52 52

4.4 Espalhamento em campo forte - efeito glória . . . . . . . . . . . . . . 53

5 Resultados 5

5.1 Métodos de cálculo . . . . . . . . . . . . . . . . . . 59 59

5.2 Seção de choque de absorção . . . . . . . . . . . . . . . . . . 61

5.2.1 Caso escalar em Reissner-Nordström . . . . . . . . . . . . . . . . 61 
5.2.2 Caso de buracos acústicos canônicos . . . . . . . . . . . . . . 64

5.2.3 Caso eletromagnético em Schwarzschild . . . . . . . . . . . . . . . . . 66

5.3 Seção de choque de espalhamento . . . . . . . . . . . . . . . 69

5.3.1 Caso escalar em Reissner-Nordström . . . . . . . . . . . . . . . . 69

5.3.2 Caso de buracos acústicos canônicos . . . . . . . . . . . . . 73

5.3.3 Caso eletromagnético em Schwarzschild . . . . . . . . . . . . . . 77

6 Conclusão 83 


\section{Lista de Figuras}

2.1 Potencial efetivo para geodésicas nulas no espaço-tempo de buracos negros

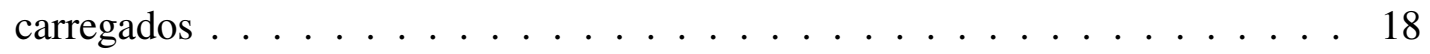

2.2 Geodésicas nulas ao redor de buracos negros de Reissner-Nordström para diferentes valores da intensidade de carga . . . . . . . . . . . . . . 20 20

2.3 Potencial efetivo de geodésicas nulas no espaço-tempo efetivo de buracos acústicos canônicos . . . . . . . . . . . . . . . . . 22

2.4 Geodésicas ao redor de buracos acústicos canônicos com parâmetros de impacto próximos ao parâmetro de impacto crítico . . . . . . . . . . . . 23

3.1 Potencial efetivo para o campo escalar não massivo ao redor de buracos negros de Reissner-Nordström extremos . . . . . . . . . . . . . . . . . . . . 27

3.2 Potencial efetivo para o campo escalar não massivo plotado com $l=0$ para diferentes valores da carga do buraco negro . . . . . . . . . . . . . 28

3.3 Potencial efetivo de perturbações ao redor de um buraco acústico canônico . . . 30

3.4 Potencial efetivo eletromagnético ao redor de buracos negros de Schwarzschild. 35

3.5 Potencial efetivo eletromagnético em Schwarzschild plotado em termos da coordenada de Wheeler. . . . . . . . . . . . . . . . . . . . . . 40 40

4.1 Seção de choque diferencial de espalhamento de buracos negros de Schwarzschild obtida com a aproximação do efeito glória . . . . . . . . . . . . 55

4.2 Ilustração do efeito glória em buracos negros de Schwarzschild . . . . . . . . . 56

5.1 Seção de choque escalar de absorção parcial de buracos negros de ReissnerNordström quando $l=0 \ldots \ldots \ldots \ldots$. . . . . . . . . . . . . . . .

5.2 Seção de choque escalar de absorção de buracos negros de Reissner-Nordström extremos ........................... 63

5.3 Seção de choque escalar total de absorção de buracos negros de ReissnerNordström para diferentes intensidades de carga . . . . . . . . . . . . . . 64

5.4 Seção de choque de absorção parcial e total de buracos acústicos canônicos . . 65

5.5 Comparação entre a seção de choque de absorção de buracos acústicos canônicos e buracos negros de Schwarzschild . . . . . . . . . . . . . . . 66 66

5.6 Seção de choque de absorção eletromagnética de buracos negros de Schwarzschild. ........................... 67 
5.7 Comparação entre a seção de choque de absorção de buracos negros de Schwarzs-

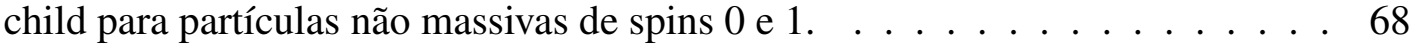

5.8 Seção de choque escalar diferencial de espalhamento de buracos negros de Reissner-Nordström em comparação com o efeito glória . . . . . . . . . . . . 70

5.9 Comparação da seção de choque diferencial de espalhamento de buracos negros de Reissner-Nordström para diferentes intensidades de carga . . . . . . . 71

5.10 Intensidade do espalhamento escalar de buracos negros de Reissner-Nordström no ângulo $180^{\circ} \ldots \ldots \ldots \ldots \ldots \ldots \ldots \ldots$

5.11 Comparação dos parâmetros para o efeito glória de Reissner-Nordström obtidos por meio analítico e numérico . . . . . . . . . . . . . . 74

5.12 Seção de choque diferencial de espalhamento de buracos acústicos canônicos. 75

5.13 Seção de choque de espalhamento de buracos acústicos canônicos no eixo de incidência como função da frequência. . . . . . . . . . . . . . . . 76

5.14 Seção de choque total e de espalhamento de buracos acústicos canônicos. . . . 78

5.15 Comparação da seção de choque diferencial de espalhamento de buracos acústicos canônicos com buracos negros de Schwarzschild . . . . . . . . . . . . 79

5.16 Seção de choque diferencial de espalhamento eletromagnético de buracos negros de Schwarzschild. . . . . . . . . . . . . . . . . . . . 80

5.17 Seção de choque diferencial de espalhamento de buracos negros de Schwarzs-

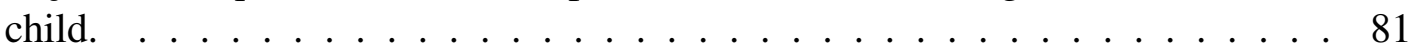




\section{Capítulo 1}

\section{Introdução}

A Relatividade Geral, elaborada por A. Einstein em 1915, é uma das mais elegantes teorias da Física e a que melhor descreve a interação gravitacional [1, 2]. Atualmente, diversos resultados e previsões desta teoria são comprovados, alguns com excelente precisão [3]. Dentre as previsões mais famosas da Relatividade Geral que puderam ser comprovadas, podemos citar o desvio da luz perante fortes campos gravitacionais e, de forma indireta, a existência de ondas gravitacionais e de buracos negros [4].

A primeira solução exata das equações de Einstein no vácuo que descreve um espaço-tempo curvo surgiu apenas alguns meses após a elaboração da Relatividade Geral. Esta solução, encontrada por K. Schwarzschild, descreve um buraco negro sem carga e sem rotação e é uma das soluções mais importantes das equações de Einstein. Os buracos negros de Schwarzschild são os buracos negros mais simples que existem, pois são caracterizados apenas pela sua massa. Em geral, no entanto, buracos negros podem conter ainda carga e/ou rotação [5].

Os buracos negros são objetos realmente singulares na natureza e o seu estudo se torna ainda mais importante e interessante em consequência da existência de diversos fenômenos que lhes são peculiares. O exemplo mais famoso destes fenômenos é a radiação Hawking [6], mas outros como a super-radiância [7] e a conversão de ondas eletromagnéticas em gravitacionais e viceversa [8]-[14] são também muito interessantes. Além dos buracos negros, estes fenômenos têm uma coisa em comum, que é o fato de estarem, de alguma forma, relacionados aos processos de absorção e espalhamento.

Devido à relação dos processos de absorção e espalhamento com os fenômenos citados acima, grande parte das pesquisas nesse contexto foi realizada na década de 70 ( $c f$. referências em [15]). Nessa época, a maior parte dos resultados era obtida de forma analítica e, portanto, válida apenas em alguns limites do problema, geralmente em baixas e altas frequências. Atualmente, muitas pesquisas ainda são desenvolvidas no contexto da absorção e do espalhamento de ondas por buracos negros. Entretanto, devido ao avanço computacional, muitas delas objetivam resultados mais gerais, válidos em qualquer limite. Exemplos disto são os resultados apresentados aqui. Uma das motivações para estas análises mais profundas do que ocorre ao redor dos buracos negros está relacionado com a sua própria existência. Hoje, acredita-se que buracos negros existam, talvez aos milhões, em quase, se não todas, as galáxias do Universo [4]. Sendo 
assim, estes objetos podem estar relacionados aos mais diversos fenômenos cosmológicos que há muito tempo são conhecidos. Portanto, entender como os buracos negros influenciam o ambiente ao seu redor é de fundamental importância.

A presente tese aborda pesquisas inseridas no contexto do estudo da absorção e do espalhamento de ondas por buracos negros e análogos. Os objetos de estudo destas pesquisas são os buracos negros de Schwarzschild, os seus análogos acústicos e os buracos negros de Reissner-Nordström. No caso de buracos negros descarregados e sem rotação, analisamos os espectros de absorção e espalhamento para os campos escalar não massivo e eletromagnético. No caso de de buracos negros estáticos e carregados, estudamos os seus espectros de absorção e espalhamento apenas para o campo escalar não massivo.

Os primeiros cálculos numéricos dos espectros de emissão, absorção e espalhamento por buracos negros de Schwarzschild foram realizados já na década de 70. Por exemplo, em 1976 D. N. Page [16] calculou numericamente a taxa de emissão de partículas não massivas de spins 0, 1/2, 1 e 2 por buracos negros de Schwarzschild válida para qualquer frequência. Em 1978, N. Sanchez calculou a seção de choque de absorção [17] e de espalhamento [18] desses buracos negros para o campo escalar não massivo, também de forma numérica. Atualmente, no caso de buracos negros de Schwarzschild, tanto a taxa de emissão, quanto a seção de choque de absorção e de espalhamento de partículas não massivas de spins $0,1 / 2,1$ e 2 são conhecidas em qualquer limite de frequência e ângulo de espalhamento [15]-[23]. Os buracos negros de Reissner-Nordström não foram tão estudados quanto os de Schwarzschild e muito ainda pode ser feito com relação ao estudo da absorção e espalhamento por estes objetos.

Uma nova área onde é possível aplicar a teoria de espalhamento, e que proporcionou maior importância ao estudo do campo escalar não massivo, surgiu em 1981, quando W. G. Unruh descobriu que é possível, em princípio, simular diversos aspectos de buracos negros em laboratório [24]. Isto se deve ao fato de que uma perturbação sobre um fluido, sem viscosidade, barotrópico e cujo fluxo é irrotacional, pode ser descrita pela equação de Klein-Gordon em uma métrica efetiva que é "percebida" apenas por esta perturbação.

O estudo do espalhamento por buracos acústicos, como são conhecidos os objetos que simulam as propriedades de buracos negros em fluidos, é muito importante, tendo em vista que o surgimento destes objetos foi motivado pela possibilidade da observação, em laboratório, de fenômenos intrínsecos aos buracos negros. Atualmente, sabe-se que é possível simular as mais diversas propriedades de espaços-tempos curvos em laboratório, e não apenas em fluidos [25]. Apesar de ser relativamente nova, muito trabalho já foi realizado no contexto da área de análogos gravitacionais, como é conhecida a área que abrange a possibilidade destas simulações. Na literatura, já pode ser encontrada um série de trabalhos que tratam de pesquisas da área de gravitação aplicadas a esta nova área. Aguns exemplos disso são os artigos sobre radiação Hawking [25, 26, 27], super-radiância (super-ressonância) [28, 29, 30, 31], modos quasinormais [32], backreaction [33, 34] e até mesmo modelos de universo [35] baseados no estudo de análogos gravitacionais.

O nosso objeto de estudo no contexto da área de análogos acústicos gravitacionais é o buraco acústico canônico [36]. Este objeto é bastante similar ao buraco negro de Schwarzschild por ser estacionário, esfericamente simétrico e possuir apenas um horizonte de eventos. Apesar 
destas similaridades, a análise do espalhamento de buracos acústicos canônicos revela pelo menos uma diferença importante em relação ao caso dos buracos negros originais: a seção de choque diferencial de espalhamento é finita para qualquer ângulo de espalhamento, o que não acontece no caso de buracos negros de Schwarzschild (e Reissner-Nordström).

Ao lidar com o campo escalar não massivo, precisamos encontrar soluções para a equação de Klein-Gordon:

$$
\square \Phi=\nabla_{\mu} \nabla^{\mu} \Phi=\frac{1}{\sqrt{-g}} \partial_{\mu}\left(\sqrt{-g} g^{\mu v} \partial_{\nu} \Phi\right)=0 .
$$

Devido aos espaços-tempos abordados aqui serem esfericamente simétricos e estacionários, as partes angular e temporal da solução desta equação são trivialmente encontradas. Já a solução da parte radial não pode ser escrita em termos de funções especiais conhecidas. Por isso, lançamos mão de métodos numéricos para encontrar resultados do problema de absorção e espalhamento válidos em qualquer limite. Apesar disso, é possível obter soluções analíticas em baixas frequências e assintoticamente, permitindo que abordemos o problema analiticamente em certos limites e usemos estes resultados analíticos em comparação com nossos resultados numéricos.

No caso do campo eletromagnético, devemos analisar as equações de Maxwell que, generalizadas para espaços-tempos curvos e sem fontes, se resumem a:

$$
\nabla_{\mu} F^{\nu \mu}=0
$$

$\mathrm{e}$

$$
\nabla_{[\mu} F_{\nu \lambda]}=0
$$

Como no caso do campo escalar não massivo, podemos separar os modos eletromagnéticos nas partes angular, radial e temporal. De modo análogo, devido ao fato de os buracos negros de Schwarzschild serem esfericamente simétricos e estacionários, as partes angular e temporal podem ser expandidas em termos de funções conhecidas. Porém, o mesmo não ocorre com a parte radial, de modo que o problema deve ser desenvolvido numericamente.

As principais aproximações analíticas apresentadas aqui são as que podem ser comparadas aos resultados numéricos. Estas são a seção de choque de absorção em baixas e altas frequências, e a seção de choque diferencial de espalhamento para ângulos próximos a $180^{\circ}$. A seção de choque de absorção em baixas frequências é obtida através do desenvolvimento analítico das partes radias das equações dos campos nesse limite. A seção de choque de absorção em altas frequências pode ser obtida através da análise de geodésicas. O estudo de geodésicas é importante também para a obtenção da seção de choque diferencial de espalhamento no limite de ângulos próximos a $180^{\circ}$. Este resultado, por sua vez, é obtido em conjunto com a fórmula do efeito glória [37], que está diretamente relacionado à órbita instável de partículas não massivas. É importante ressaltar que nossos resultados estão em excelente concordância com estas aproximações, o que nos permite concluir que os nossos cálculos numéricos são bastante precisos.

O presente texto está dividido da seguinte maneira: No Capítulo 2 descrevemos os espaçostempos estudados dando ênfase à análise de geodésicas nulas, importantes para algumas aproximações usadas aqui. No Capítulo3 desenvolvemos as equações de Klein-Gordon e de Maxwell, 
as mais importantes deste estudo. Lá, obteremos soluções assintóticas e os coeficientes relacionados ao espalhamento. No Capítulo 4 mostramos as definições e algumas aproximações importantes das seções de choque de absorção e espalhamento. No Capítulo 5 apresentamos nossos resultados. Nesse capítulo descrevemos também os principais métodos utilizados nesta pesquisa. Finalmente, no Capítulo 6 apresentamos nossas conclusões e considerações finais.

No decorrer desta tese adotaremos o sistema de unidades naturais, no qual $G=c=\hbar=1$, a não ser quando especificado de outra forma. No caso especial de buracos acústicos canônicos, a velocidade que encontramos nas equações é a velocidade da perturbação no fluido, que aqui chamaremos de $c_{\text {som. }}$. Por simplicidade, faremos $c_{\text {som }}=1$ quando estivermos tratando destes objetos. 


\section{Capítulo 2}

\section{Os espaços-tempos}

No presente capítulo apresentamos uma análise dos espaços-tempos tratados neste trabalho. Aqui nos restringimos puramente aos resultados baseados nas equações para geodésicas. Basicamente, a partir do estudo de geodésicas destes espaços-tempos, obtemos alguns resultados analíticos que serão usados para comparação com os resultados numéricos e também para melhor compreensão do problema de espalhamento por buracos negros. Como devemos esperar, resultados obtidos através do estudo de geodésicas são válidos apenas para frequências muito altas. Com a análise mostrada aqui é possível conhecermos a seção de choque de absorção em altas frequências, além de obtermos dados necessários para a aproximação da seção de choque diferencial de espalhamento em ângulos de $180^{\circ}$ (efeito glória).

\subsection{Buracos negros de Reissner-Nordström}

Buracos negros carregados e sem rotação, ou buracos negros de Reissner-Nordström, são descritos pelo seguinte elemento de linha:

$$
d s^{2}=f(r) d t^{2}-f(r)^{-1} d r^{2}-r^{2}\left(d \theta^{2}+\operatorname{sen}^{2} \theta d \phi^{2}\right),
$$

no qual

$$
f(r)=1-\frac{2 M}{r}+\frac{Q^{2}}{r^{2}}
$$

sendo $M$ e $Q$ a massa e a carga do buraco negro, respectivamente [5, 38]. Estes buracos negros possuem dois horizontes de eventos, que são determinados por $r_{ \pm}=M \pm \sqrt{M^{2}-Q^{2}}$. Note ainda que

$$
f=\left(1-\frac{r_{+}}{r}\right)\left(1-\frac{r_{-}}{r}\right)
$$

Aqui nos deteremos basicamente ao estudo de buracos negros com três valores da razão "intensidade de carga/massa do buraco negro" $(q=|Q| / M)$ :

(1) $q=0 \Longrightarrow$ Buraco negro sem carga e sem rotação. Este é o buraco negro de Schwarzschild. Trata-se de uma das soluções mais simples e importantes das equações de Einstein. A 
maior parte dos estudos de buracos negros trata de buracos negros de Schwarzschild. Por exemplo, tanto a taxa de emissão [16], como a seção de choque de absorção [22] e diferencial de espalhamento [23], válidas em qualquer limite, são conhecidas para partículas não massivas de spin $0,1 / 2,1$ e 2. Entretanto, nem todos estes resultados são conhecidos para os casos de buracos negros de Reissner-Nordström ou Kerr. No caso de buracos negros de Schwarzschild temos $r_{+}=r_{\mathrm{S}}=2 M$ e $r_{-}=0$;

(2) $q=0.8 \Longrightarrow$ Buracos negros de Reissner-Nordström "típicos". Nesse caso, assim como todos outros em que $0<q<1$, os dois horizontes de eventos são distintos $\left(r_{+}=1.6 \mathrm{M}\right.$ e $r_{-}=0.4 M$ nesse caso específico). Vale ressaltar que o estudo descrito aqui e nossos cálculos podem ser usados para reproduzir resultados com qualquer valor de $0 \leq q \leq 1$;

(3) $q=1 \Longrightarrow$ Buracos negros com este valor da carga são buracos negros extremamente carregados 1 . Nesse caso temos $r_{+}=r_{-}=M$.

Diferentemente de buracos negros de Schwarzschild, e até mesmo de buracos negros de Kerr [15], o espalhamento de buracos negros de Reissner-Nordström não foi tão estudado. Apesar disso, o estudo destes buracos negros é muito importante, pois nos permite entender ainda mais como as características de espalhamento mudam com a mudança das condições de contorno do problema.

Dadas estas propriedades gerais do espaço-tempo de Reissner-Nordström, podemos iniciar a análise de geodésicas neste espaço-tempo. Começamos usando 2.11 para escrever

$$
\dot{s}^{2}=f \dot{t}^{2}-f^{-1} \dot{r}^{2}-r^{2}\left(\dot{\theta}^{2}+\dot{\phi}^{2} \operatorname{sen}^{2} \theta\right)=\kappa,
$$

onde o ponto em cima das coordenadas representa diferenciação com relação a um parâmetro afim e $\kappa=0,+1,-1$ determina se as geodésicas são do tipo luz (também chamadas geodésicas nulas) tipo tempo ou tipo espaço, respectivamente.

Aqui investigaremos as geodésicas de duas formas: (i) analisando somente a parte radial do movimento das partículas (para determinar a seção de choque de absorção em altas frequências) e (ii) encontrando $r$ como função de $\phi$, de modo que as formas das geodésicas possam ser diretamente obtidas (um dos passos necessários para análise do efeito glória). Na verdade, através da análise (ii) podemos obter todas a informações que necessitamos, mas a análise (i) traz uma forma mais eficiente de entendermos a seção de choque de absorção em altas frequências.

Espaços-tempos de Reissner-Nordström possuem dois campos de Killing [1] importantes para o cálculo da seção de choque em altas frequências. Estes campos de Killing são $\xi^{\mu}=$ $\left(\partial_{t}\right)^{\mu}=(1,0,0,0)$ e $\zeta^{\mu}=\left(\partial_{\phi}\right)^{\mu}=(0,0,0,1)$. Com estes campos encontramos as constantes de movimento

$$
E=g_{\mu \nu} \xi^{\mu} \frac{d x^{\nu}}{d \tau}=f \dot{t}
$$

\footnotetext{
${ }^{1}$ Para $|Q|>M$, a principal característica de um buraco negro, que é o horizonte de eventos, é perdida. Nesse caso, não existe um buraco negro, mas uma singularidade nua [1, 2, 39].
} 
e

$$
L=g_{\mu \nu} \zeta^{\mu} \frac{d x^{\nu}}{d \tau}=r^{2} \dot{\phi}
$$

nas quais $\tau$ é o parâmetro afim das geodésicas em questão.

Em se tratando de um espaço-tempo esfericamente simétrico, uma geodésica qualquer estará restrita a um plano. Considerando o plano $\theta=\pi / 2$ na equação (2.4), e usando as equações (2.5) e (2.6), obtemos:

$$
\frac{E^{2}}{2}=U_{\mathrm{RN}}(r)+\frac{\dot{r}^{2}}{2}
$$

na qual

$$
U_{\mathrm{RN}}(r)=\frac{L^{2} f}{2 r^{2}}
$$

A equação (2.7) tem a mesma forma da equação radial que descreve o movimento de uma partícula sujeita a um potencial central no formalismo da Mecânica Clássica [40]. No presente caso porém, o potencial efetivo é $U_{\mathrm{RN}}$. Na Figura 2.1 mostramos este potencial efetivo para alguns valores da razão $q$. Lá é possível visualizar que este potencial vai a zero no horizonte de eventos e no infinito. Podemos ver também que a sua intensidade aumenta e que o valor da coordenada radial do ponto de máximo diminui à medida que aumentamos a intensidade da carga do buraco negro.

Existem três configurações possíveis a serem analisadas com a equação de geodésica (2.7) (abaixo, $U_{\mathrm{RN}}^{\max }$ é o máximo do potencial efetivo $U_{\mathrm{RN}}$ ):

(1) $E^{2} / 2<U_{\mathrm{RN}}^{\max } \Longrightarrow$ Nesse caso, partículas provenientes do infinito teriam velocidade radial não nula enquanto $E^{2} / 2>U_{\mathrm{RN}}$. Quando $E^{2} / 2=U_{\mathrm{RN}}$ a partícula pararia (radialmente) e iniciaria o processo de retorno ao infinito.

(2) $E^{2} / 2=U_{\mathrm{RN}}^{\max } \Longrightarrow$ Se isso ocorrer, a partícula vem "caindo" do infinito, mas quando chega ao ponto onde o potencial é máximo, sua velocidade radial é zero, ou seja $\dot{r}=0$, como podemos concluir da equação (2.7). No ponto onde o potencial é máximo, existe então uma órbita instável para geodésicas nulas. Nesse caso a partícula giraria em volta do buraco negro um número infinito de vezes.

(3) $E^{2} / 2>U_{\mathrm{RN}}^{\max } \Longrightarrow$ Nesse caso, a partícula que vem do infinito passa o máximo do potencial e segue até atravessar o horizonte de eventos mais externo do buraco negro. Uma vez que isso acontece, a partícula não pode mais sair do buraco negro e portanto é absorvida.

Dentro destes três casos, o caso (2) é um limite entre (1) e (3). Resumidamente, partículas com $E^{2} / 2<U_{\mathrm{RN}}^{\max }$ serão espalhadas enquanto partículas com $E^{2} / 2>U_{\mathrm{RN}}^{\max }$ serão absorvidas pelo buraco negro. O caso limite nos define as condições inicias para que a partícula seja ou não absorvida. Analisando $E^{2} / 2=U_{\mathrm{RN}}^{\max }$ podemos escrever:

$$
b_{\mathrm{c}}=\frac{L}{E}=r_{\max }\left(1-\frac{2 M}{r_{\max }}+\frac{Q^{2}}{r_{\max }^{2}}\right)^{-1 / 2},
$$




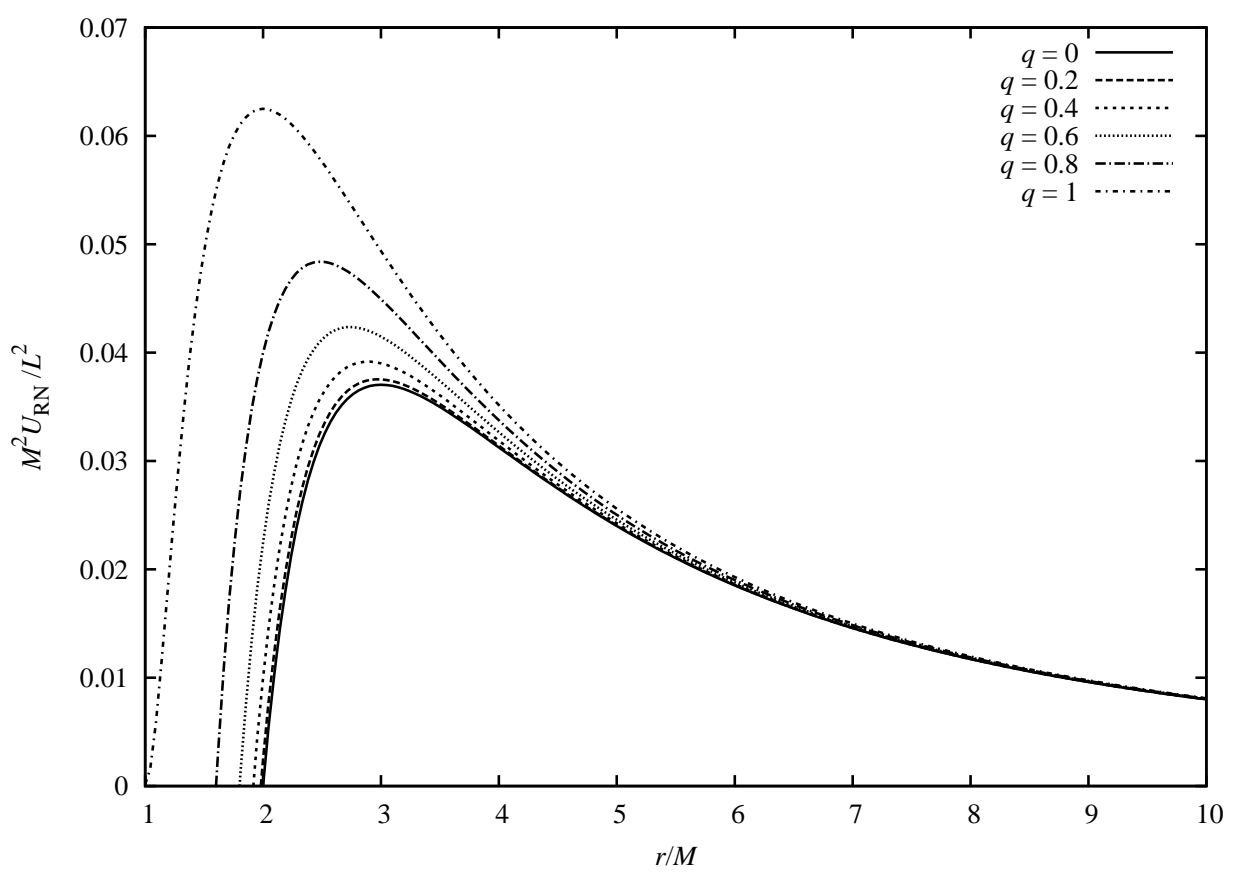

Figura 2.1: Potencial efetivo para geodésicas nulas no espaço-tempo de Reissner-Nordström. Aqui os valores das intensidades de carga $q$ são $0,0.2,0.4,0.6,0.8$ e 1 . Note que a intensidade do potencial aumenta quando aumentamos a carga do buraco negro. Além disso, ele vai a zero no horizonte de eventos e no infinito. É importante perceber também que a posição radial do ponto de máximo deste potencial diminui com o aumento da intensidade de carga.

sendo que $r_{\max }$ é o valor da coordenada radial para qual o potencial efetivo é máximo. Esta equação define o parâmetro de impacto crítico $b_{c}$ para geodésicas nulas ${ }^{2}$. Evidentemente, geodésicas com $b>b_{\mathrm{c}}$ serão espalhadas enquanto que aquelas com $b<b_{\mathrm{c}}$ serão absorvidas. Nesse caso, vemos que $b_{c}$ é o raio de uma seção transversal circular onde qualquer geodésica dentro dela tem seu fim no buraco negro. Portanto, a seção de choque de absorção em altas frequências é definida como $\sigma_{\text {abs }}^{\text {af }}=\pi b_{\mathrm{c}}^{2}$.

Calculando $r_{\max }$, obtemos que [41]

$$
\sigma_{\mathrm{abs}}^{\mathrm{RN}-\mathrm{af}}=\pi \frac{\left(3 M+\sqrt{9 M^{2}-8 Q^{2}}\right)^{4}}{8\left(3 M^{2}-2 Q^{2}+M \sqrt{9 M^{2}-8 Q^{2}}\right)} .
$$

Fazendo $Q=0$, obtemos que $\sigma_{\text {abs }}^{\mathrm{RN}-\mathrm{af}}=27 \pi M^{2}=(27 / 4) \pi r_{+}^{2}$ (relembramos que $r_{+}=2 M$ e $r_{-}=$ 0 nesse caso) que é um resultado bastante conhecido para buracos negros de Schwarzschild [1, 42]. No caso de buracos negros de Reissner-Nordström extremos, $Q^{2}=M^{2}$, obtemos que $\sigma_{\mathrm{abs}}^{\mathrm{RN}-\mathrm{af}}=16 \pi M^{2}=16 \pi r_{+}^{2}$ (relembramos que $r_{+}=r_{-}=M$ no caso extremo). No Capítulo 5 ,

\footnotetext{
${ }^{2}$ Por definição, o parâmetro de impacto aparente é $b=L / E[1]$.
} 
usaremos estes valores para comparação com os resultados numéricos que obteremos para a seção de choque de absorção de buracos negros de Reissner-Nordström.

Outra forma de abordar geodésicas nulas, é relacionar a coordenada radial com a coordenada polar $\phi$. Nesse caso, podemos utilizar as equações (2.4), (2.5) e (2.6) para obter a equação de órbita para geodésicas nulas:

$$
\left(\frac{d u}{d \phi}\right)^{2}=\frac{1}{b^{2}}-u^{2}+2 M u^{3}-Q^{2} u^{4}
$$

sendo $u=1 / r$. Derivando esta equação em reação a $\phi$ obtemos:

$$
\frac{d^{2} u}{d \phi^{2}}+u=3 M u^{2}-2 Q^{2} u^{3}
$$

Por meio das equações (2.11) e 2.12) é possível obter a forma explícita das geodésicas nulas em Reissner-Nordström. Algumas dessas geodésicas são mostradas na Figura 2.2. Nesta figura escolhemos o parâmetro de impacto $b=5.2 \mathrm{M}$ e diferentes valores de $q$. Note que, enquanto geodésicas com $b=5.2 M$ sofrem deflexão de mais de $360^{\circ}$ no espaço-tempo de Schwarzschild, geodésicas com o mesmo parâmetro de impacto sofrem deflexões em torno de $180^{\circ}$ para buracos negros com $q=0.4$ e em torno de $90^{\circ}$ para buracos negros de Reissner-Nordström extremos. Isto está de acordo com o fato de a seção de choque de absorção (no limite de altas frequências) diminuir com o aumento da intensidade da carga do buraco negro [equação (2.10)].

Para tratar o efeito glória é importante que conheçamos o ângulo de deflexão como função do parâmetro de impacto. Apesar de a fórmula do efeito glória não ser clássica (como veremos na seção 4.4), os parâmetros presentes nela podem ser obtidos através da análise de geodésicas [15]. Com este objetivo, integramos a equação (2.11) para obter o ângulo de deflexão

$$
\Theta(b)=\frac{4}{\sqrt{Q^{2}\left(u_{3}-u_{1}\right)\left(u_{2}-u_{0}\right)}}[K(k)-F(z, k)]-\pi,
$$

na qual $K(k)$ e $F(z, k)$ são integrais elípticas completas e incompletas de primeiro tipo, respectivamente [43], com

$\mathrm{e}$

$$
k^{2}=\frac{\left(u_{3}-u_{2}\right)\left(u_{1}-u_{0}\right)}{\left(u_{3}-u_{1}\right)\left(u_{2}-u_{0}\right)},
$$

$$
z=\left[-\frac{u_{0}\left(u_{3}-u_{1}\right)}{u_{3}\left(u_{1}-u_{0}\right)}\right]^{1 / 2} \text {. }
$$

Além disso, $u_{0}, u_{1}=1 / r_{\min }, u_{2}$ e $u_{3}$ são raízes da equação

$$
\frac{1}{b^{2}}-u^{2}+2 M u^{3}-Q^{2} u^{4}=0
$$

que corresponde a $(d u / d \phi)^{2}=0$ [cf. eq. [2.11] ], com $r_{\min }$ sendo a distância mínima entre a geodésica e o buraco negro. Para geodésicas de espalhamento, ou seja, aquelas que começam e terminam no infinito, devemos ter $u_{0}<0$ e $u_{3}>u_{2} \geq u_{1}>0$.

A análise que necessitamos para a obtenção do efeito glória pode ser feita resolvendo (2.13) numericamente ou através de aproximações analíticas das integrais elípticas. 


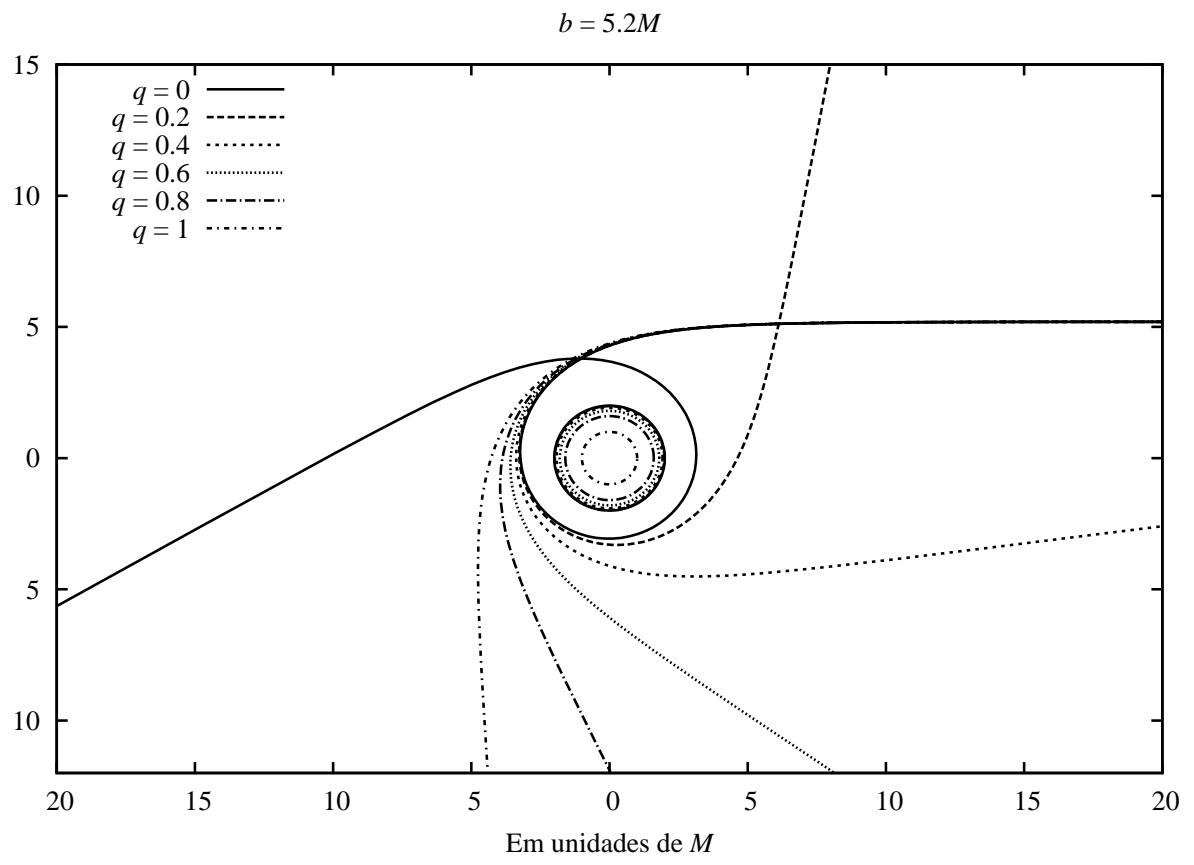

Figura 2.2: Geodésicas nulas ao redor de buracos negros de Reissner-Nordström para $b=5.2 M$ e com diferentes valores da intensidade de carga. Vemos que quanto maior a intensidade da carga do buraco negro, menos curvada é a geodésica. Enquanto que no caso de Schwarzschild a geodésica dá mais de uma volta ao redor do buraco negro, no caso extremo a geodésica muda de direção em torno de apenas $90^{\circ}$. Os círculos representam os horizontes de eventos em cada caso. 


\subsection{Buracos acústicos canônicos}

Buracos acústicos canônicos são formados por fluidos incompressíveis cujo fluxo é estacionário e esfericamente simétrico. Nesse caso, a equação da continuidade requer que a velocidade do fluxo seja $v_{r}=r_{c}^{2} / r^{2}$, sendo que $r_{c}$ é a distância da origem ao ponto no qual a velocidade do fluxo excede a velocidade do som no fluido.

A idéia subjacente aos buracos acústicos é que uma perturbação sobre o fluido, $\delta \vec{v}=-\nabla \Phi$, pode ser descrita pela equação de Klein-Gordon, (1.1). No caso de buracos acústicos canônicos, a métrica efetiva para perturbações no fluido pode ser dada por [36]:

$$
d s^{2}=h(r) d t^{2}-h(r)^{-1} d r^{2}-r^{2}\left(d \theta^{2}+\operatorname{sen}^{2} \theta d \phi^{2}\right),
$$

onde

$$
h(r)=1-\frac{r_{\mathrm{c}}^{4}}{r^{4}}
$$

Como podemos observar, a métrica efetiva de buracos acústicos canônicos é muito semelhante à métrica de buracos negros de Schwarzschild [obtida fazendo-se $Q=0 \mathrm{em}(2.1)-(2.2)]$. Da mesma forma, este espaço-tempo efetivo é estacionário e esfericamente simétrico. $\mathrm{O}$ horizonte de eventos do buraco acústico canônico é a superfície de raio $r_{\mathrm{c}}$, como deveríamos esperar, uma vez que nesta superfície a velocidade do fluxo se iguala à velocidade do som no fluido.

Apesar de as considerações acima não serem válidas para frequências muito altas 3 [44], é muito útil analisarmos geodésicas nulas na métrica de buracos acústicos canônicos. Assim como no caso de buracos negros de Reissner-Nordström, os resultados vão servir para comparação com os resultados numéricos da seção de choque de absorção e, principalmente, na obtenção de valores para o efeito glória.

Como procedemos para o caso de buracos negros de Reissner-Nordström na seção anterior, o elemento de linha de buracos acústicos canônicos (2.14) pode ser usado para escrever

$$
\dot{s}^{2}=h \dot{t}^{2}-h^{-1} \dot{r}^{2}-r^{2}\left(\dot{\theta}^{2}+\dot{\phi}^{2} \operatorname{sen}^{2} \theta\right)=0 .
$$

Assim como buracos negros de Reissner-Nordström, os espaços-tempos efetivos de buracos acústicos canônicos possuem campos de Killing que permitem construir as constantes $E=h \dot{t}$ e $L=r^{2} \dot{\phi}$. Com estas constantes de movimento, a equação 2.16 pode ser escrita como:

$$
\frac{E^{2}}{2}=U_{\mathrm{c}}+\frac{\dot{r}^{2}}{2}
$$

onde usamos que as geodésicas estão restritas a um plano $(\theta=\pi / 2)$. O potencial efetivo é dado por:

$$
U_{\mathrm{c}}=\frac{h L^{2}}{2 r^{2}} .
$$

\footnotetext{
${ }^{3}$ As equações para as perturbações são obtidas através das equações de Euler e da continuidade, basicamente. Estas equações consideram o fluido como algo contínuo. Quando os comprimentos de onda das perturbações são da ordem das distâncias intermoleculares do fluido estas equações não serão mais válidas.
} 
Este potencial efetivo está plotado na Figura 2.3. Podemos perceber que o seu comportamento é similar ao potencial efetivo para geodésicas nulas no espaço-tempo de Reissner-Nordström (Figura 2.1). Ele é zero no horizonte de eventos e vai a zero quando $r \rightarrow \infty$.

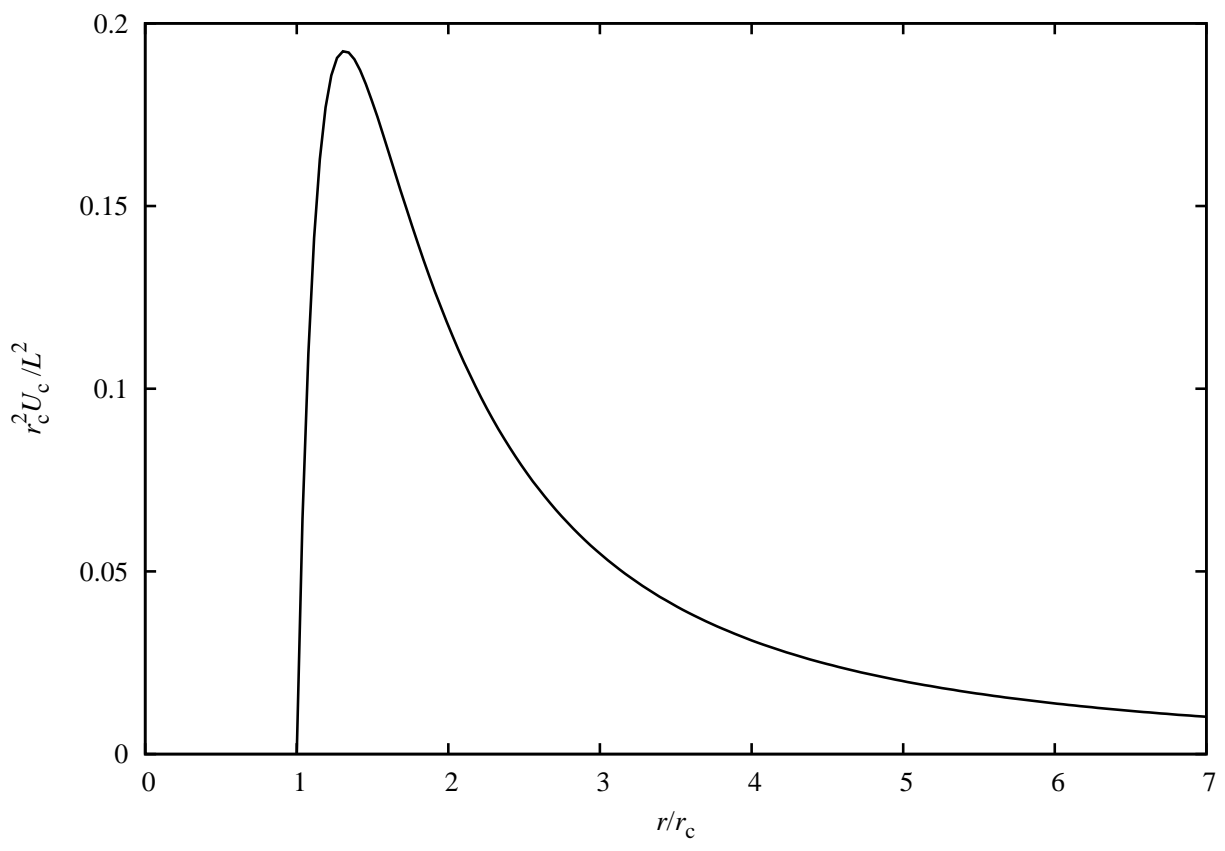

Figura 2.3: Potencial efetivo de geodésicas nulas no espaço-tempo efetivo de buracos acústicos canônicos. Aqui vemos que este potencial vai a zero no horizonte de eventos e no infinito. $\mathrm{O}$ comportamento deste potencial é muito similar ao comportamento do potencial efetivo para geodésicas nulas no espaço-tempo de Reissner-Nordström, mostrado na Figura 2.1. Assim como no caso de buracos negros estáticos e carregados, aqui existe um máximo de potencial, o que resulta na existência de uma órbita instável.

A presença de um máximo no potencial efetivo (2.18) implica na existência de uma órbita instável para geodésicas nulas no espaço-tempo efetivo de buracos acústicos canônicos. Este máximo deve ser superado por $E^{2} / 2$ para que haja absorção. Assim, obtemos que o parâmetro de impacto crítico é dado por:

$$
b_{\mathrm{c}}=r_{\max }\left(1-\frac{r_{\mathrm{c}}^{4}}{r_{\max }^{4}}\right)^{-1 / 2},
$$

onde $r_{\max }$ corresponde ao ponto de máximo do potencial efetivo (2.18). Encontrando este ponto, obtemos a seção de choque de absorção de buracos acústicos canônicos em altas frequências, que é

$$
\sigma_{\mathrm{abs}}^{\mathrm{c}-\mathrm{af}}=\pi b_{\mathrm{c}}^{2}=\frac{3 \sqrt{3}}{2} \pi r_{\mathrm{c}}^{2} .
$$

Ainda através de geodésicas, podemos obter algumas informações importantes para o efeito 
glória. Nesse caso, usamos a equação $2.16(\operatorname{com} \theta=\pi / 2)$ para escrever:

$$
\left(\frac{d u}{d \phi}\right)^{2}=\frac{1}{b^{2}}-u^{2}+r_{\mathrm{c}}^{4} u^{6},
$$

onde, novamente, $u=1 / r$. Derivando ainda esta equação com relação a $\phi$ obtemos:

$$
\frac{d^{2} u}{d \phi^{2}}+u=3 r_{\mathrm{c}}^{4} u^{5}
$$

Na Figura 2.4 mostramos um conjunto de geodésicas na métrica efetiva de buracos acústicos canônicos que têm parâmetros de impacto com valores próximos ao parâmetro de impacto crítico. Podemos ver que uma das geodésicas possui $b<b_{\mathrm{c}}$, sendo $b_{\mathrm{c}}$ dado por (2.19), e por isso termina dentro do buraco mudo. As outras são iniciadas com $b>b_{\mathrm{c}}$ e por isso tem início e fim no infinito. As curvas desta figura foram obtidas por meio da solução numérica da equação (2.22).

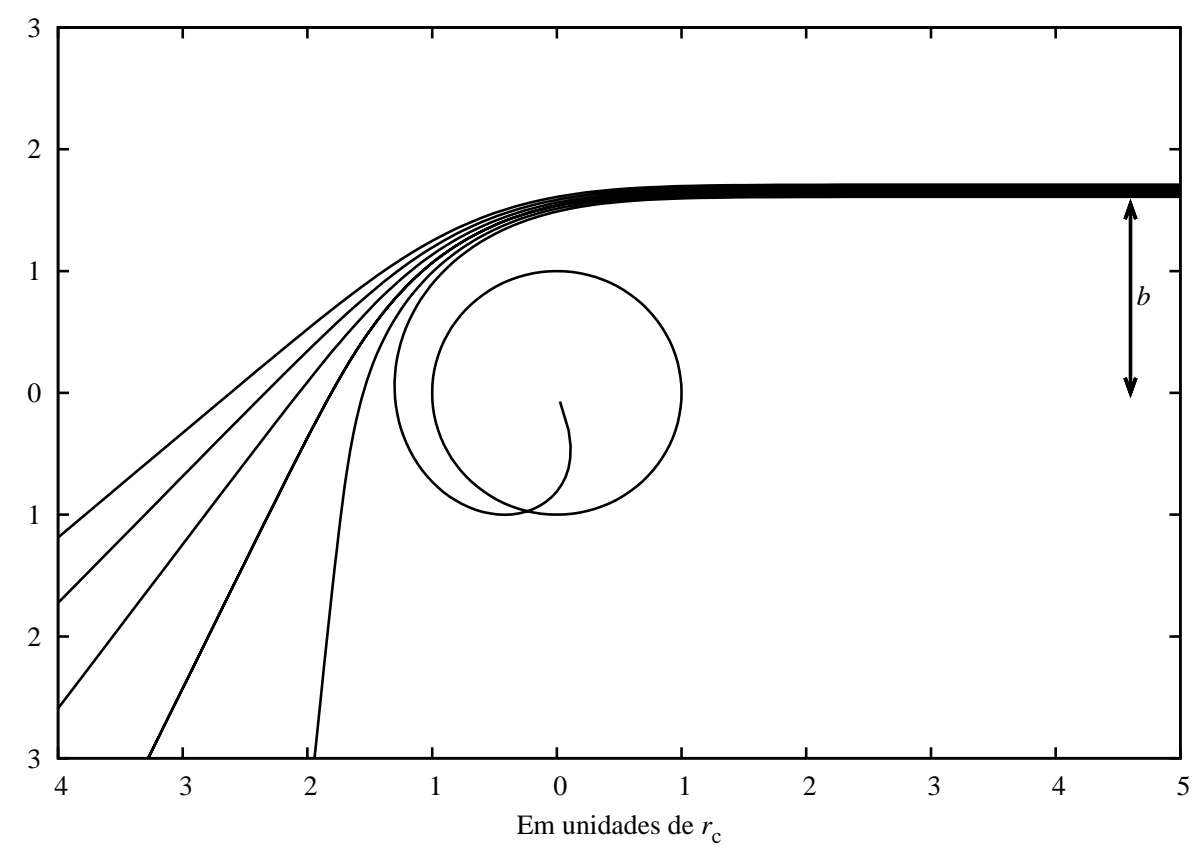

Figura 2.4: Geodésicas ao redor de buracos acústicos canônicos com parâmetros de impacto próximos ao parâmetro de impacto crítico. Pelo menos uma destas geodésicas tem fim dentro do buraco, o que caracteriza a absorção em altas frequências. Aqui, o círculo representa o horizonte de eventos.

Para obter parâmetros para o efeito glória, integramos a equação 2.21] e obtemos que o ângulo de deflexão em função do parâmetro de impacto é

$$
\Theta(b)=\frac{2 K(k)}{\sqrt{\left(v_{1}-v_{2}\right) v_{3}}}-\pi
$$


no qual

$$
k^{2}=\frac{v_{2}\left(v_{3}-v_{1}\right)}{v_{3}\left(v_{2}-v_{1}\right)},
$$

$\operatorname{com} v_{1}, v_{2}$ e $v_{3}$ sendo raízes da equação

$$
r_{\mathrm{c}}^{4} v^{3}-v+\frac{1}{b^{2}}=0
$$

Para trajetórias de espalhamento, temos $v_{1}<0$ e $v_{3}>v_{2}>0$. Da equação (2.23) podemos obter os parâmetros $b(\Theta=\pi)$ e $d b /\left.d \Theta\right|_{\Theta=\pi}$. Estes parâmetros são importantes para o estudo do efeito glória, como veremos na seção 4.4 . 


\section{Capítulo 3}

\section{Os campos}

No presente capítulo é apresentado o tratamento do comportamento dos campos estudados neste trabalho. Aqui nosso principal objetivo é obter soluções para a parte radial da equação de Klein-Gordon sujeita às métricas de Reissner-Nordström e de buracos acústicos canônicos e das equações de Maxwell ao redor de buracos negros de Schwarzschild. Estas equações radias são importantes devido à decomposição da onda espalhada em ondas parciais. A parte radial destas equações não possui soluções que possam ser expressas em termos de funções especiais conhecidas. Por isso, aqui nos limitamos a soluções assintóticas, que são importantes para a análise do espalhamento. No entanto, além das formas assintóticas destas equações, é necessário obtermos soluções gerais para chegarmos às seções de choque de absorção e espalhamento que sejam válidas em qualquer frequência. Estas soluções, válidas em qualquer limite, são obtidas através de um método numérico que é descrito na seção 5.1

\subsection{Campo escalar não massivo em Reissner-Nordström}

No caso do campo escalar não massivo, a idéia inicial é aplicar o método de separação de variáveis na equação (1.1). Utilizamos o fato de que o espaço-tempo ao redor de buracos negros de Reissner-Nordström é estacionário e esfericamente simétrico. Isso significa que a função $\Phi$ pode ser escrita como

$$
\Phi=\frac{\psi_{\omega l}(r)}{r} Y_{l m}(\theta, \phi) e^{-i \omega t},
$$

em que $Y_{l m}(\theta, \phi)$ são os harmônicos esféricos escalares. Sendo assim, a única função a ser determinada é a função radial $\psi_{\omega l}$ que obedece à seguinte equação:

$$
f \frac{d}{d r}\left[f \frac{d}{d r} \psi_{\omega l}(r)\right]+\left[\omega^{2}-V_{\mathrm{RN}}(r)\right] \psi_{\omega l}(r)=0,
$$

onde

$$
V_{\mathrm{RN}}(r)=\left(1-\frac{2 M}{r}+\frac{Q^{2}}{r^{2}}\right)\left[\frac{2 M}{r^{3}}-\frac{2 Q^{2}}{r^{4}}+\frac{l(l+1)}{r^{2}}\right] .
$$


Para tratar as soluções em regiões assintóticas, nós usamos a "coordenada tartaruga" definida como:

$$
r_{*}=r+\frac{r_{+}^{2}}{r_{+}-r_{-}} \ln \left|r-r_{+}\right|-\frac{r_{-}^{2}}{r_{+}-r_{-}} \ln \left|r-r_{-}\right| .
$$

Como casos particulares, esta coordenada se torna

$$
r_{*}=r+2 M \ln |r-2 M|
$$

em Schwarzschild (nesse caso ela é normalmente chamada de coordenada de Wheeler), e

$$
r_{*}=r+2 M \ln |r-M|-\frac{M^{2}}{r-M}
$$

no caso extremo. Em termos desta coordenada, a equação (3.2) se torna

$$
\frac{d^{2}}{d r_{*}^{2}} \psi_{\omega l}\left(r_{*}\right)+\left(\omega^{2}-V_{\mathrm{RN}}\right) \psi_{\omega l}\left(r_{*}\right)=0
$$

que se assemelha a uma equação de Schrödinger unidimensional e independente do tempo [45]. Portanto, $V_{\mathrm{RN}}$ é o potencial efetivo que determina a dinâmica do campo escalar não massivo ao redor de buracos negros de Reissner-Nordström. Plotamos alguns exemplos deste potencial nas Figuras 3.1 e 3.2, mudando os valores de carga e do número quântico $l$. Podemos concluir destas figuras que o potencial efetivo aumenta quando aumentamos $q$ ou $l$. Além disso, ele vai a zero nos limites $r \rightarrow r_{+}\left(r_{*} \rightarrow-\infty\right)$ e $r \rightarrow \infty\left(r_{*} \rightarrow \infty\right)$.

A Figura 3.2 deixa claro que, assim como o comportamento do potencial efetivo clássico, mostrado na Figura 2.1, o potencial efetivo (3.3) aumenta com a carga do buraco negro.

Como o potencial efetivo vai a zero no horizonte de eventos, podemos concluir que em regiões externas extremamente próximas ao horizonte de eventos 1

$$
\psi_{\omega l}\left(r_{*}\right) \approx A_{\omega l}^{\mathrm{tr}} e^{-i \omega r_{*}} .
$$

No caso em que $r \gg r_{+}$, o termo dominante no potencial efetivo é o termo contendo o momento angular, ou seja, o termo proporcional à $l(l+1) / r^{2}$. Nesse caso, a solução para 3.7) é

$$
\psi_{\omega l}\left(r_{*}\right) \approx \omega r_{*}\left[(-i)^{l+1} A_{\omega l}^{\mathrm{in}} h_{l}^{(1) *}\left(\omega r_{*}\right)+i^{l+1} A_{\omega l}^{\mathrm{ref}} h_{l}^{(1)}\left(\omega r_{*}\right)\right],
$$

na qual $h_{l}^{(1)}$ são as funções esféricas de Hankel ou funções esféricas de Bessel de terceiro tipo [43]. Sabendo ainda que $h_{n}^{(1)} \approx(-i)^{n+1} e^{i x} / x$ quando $x \gg n(n+1) / 2$ [46], podemos escrever que

$$
\psi_{\omega l}\left(r_{*}\right) \approx A_{\omega l}^{\mathrm{in}} e^{-i \omega r_{*}}+A_{\omega l}^{\mathrm{ref}} e^{i \omega r_{*}},
$$

quando $\omega r_{*} \gg l(l+1) / 2$.

\footnotetext{
${ }^{1}$ Note que podem haver dois tipos de soluções que estão relacionadas a modos provenientes do horizonte e modos provenientes do infinito. Como estamos analisando espalhamento, lidaremos apenas com soluções de modos provenientes do infinito.
} 


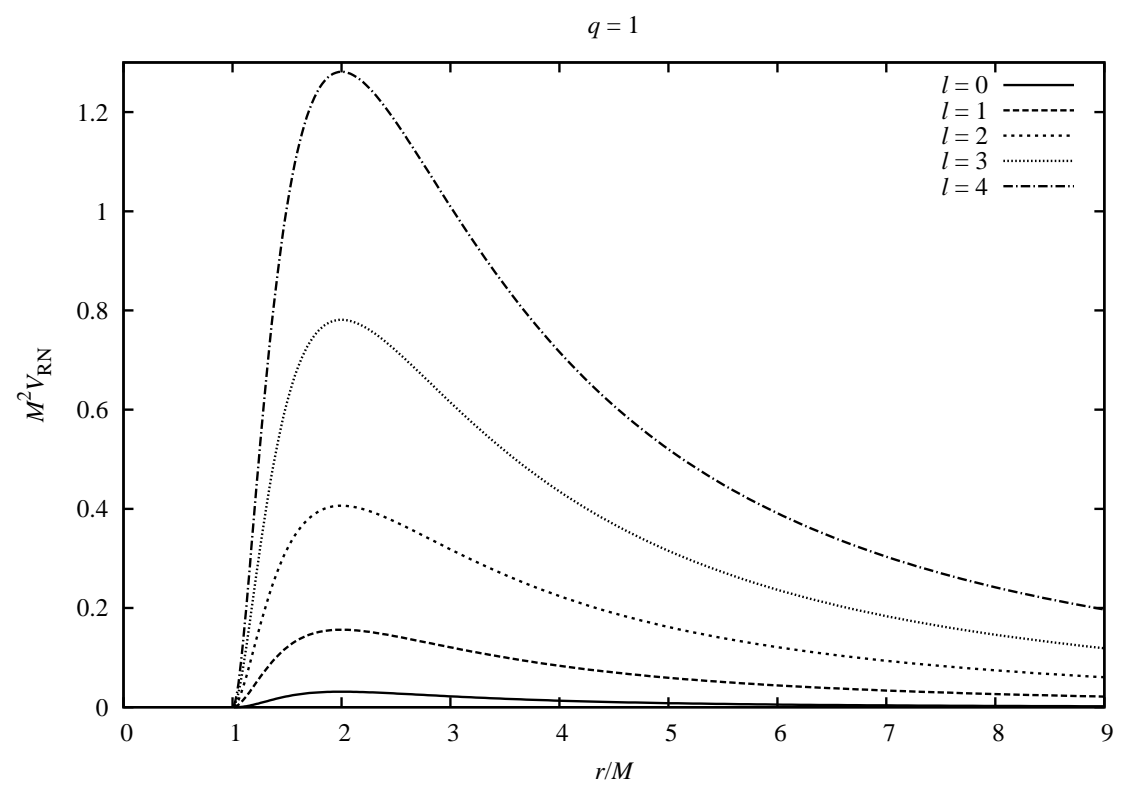

(a) Potencial efetivo para o campo escalar não massivo ao redor de buracos negros de Reissner-Nordström extremos. Aqui, temos $l$ de 0 a 4.

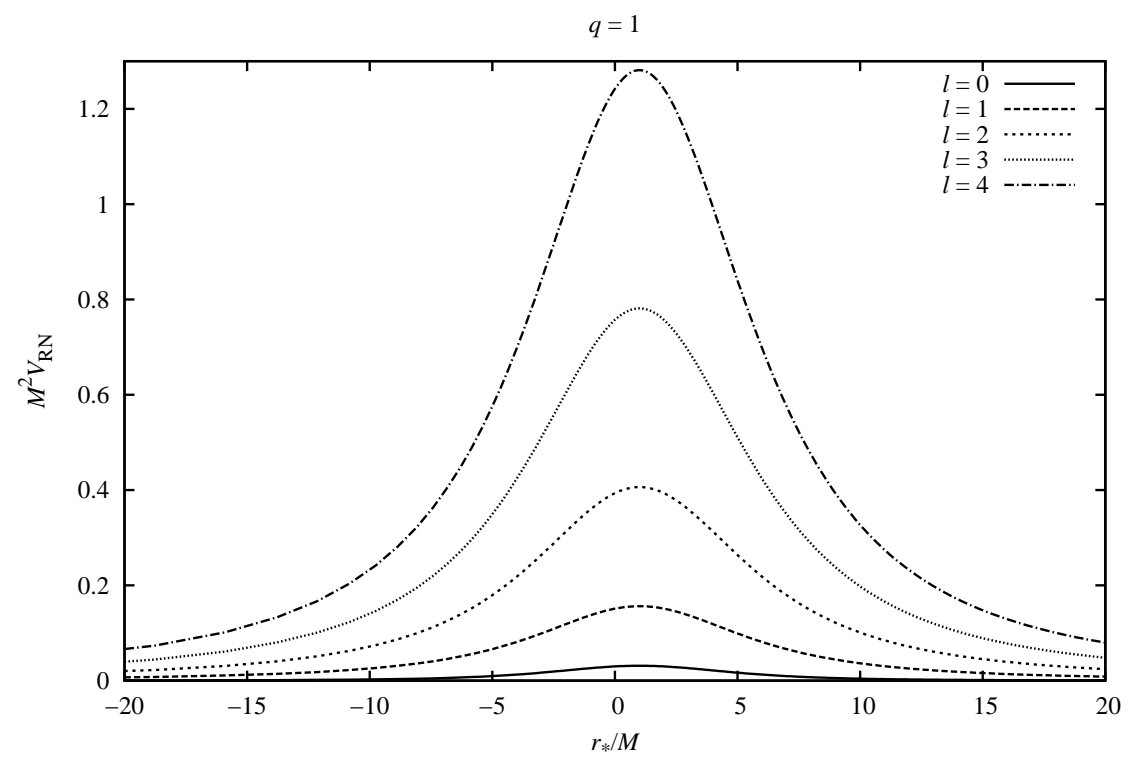

(b) Aqui temos o mesmo potencial de (a), porém como função de $r_{*}$.

Figura 3.1: Vemos que o potencial efetivo vai a zero no horizonte de eventos do buraco negro $\left(r_{*} \rightarrow-\infty\right)$ e no infinito $\left(r_{*} \rightarrow+\infty\right)$. Quando $r \gg M$, a maior contribuição para este potencial é $l(l+1) / r^{2}$. A análise deste comportamento nos permite encontrar soluções assintóticas para a equação (3.7). Notamos também que quando o número quântico $l$ cresce, este potencial também cresce. 


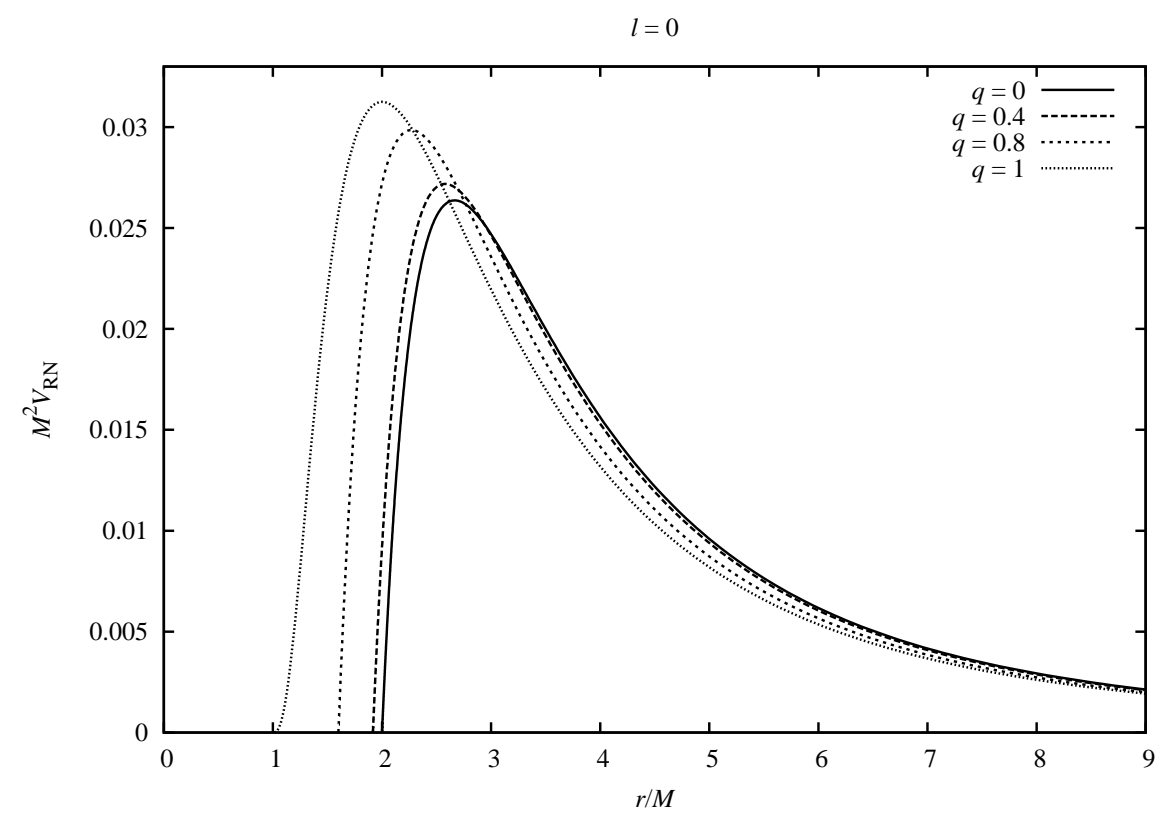

(a) Potencial efetivo para o campo escalar não massivo quando $l=0$ para buracos negros de Reissner-Nordström com $q=0,0.4,0.8$ e 1 .

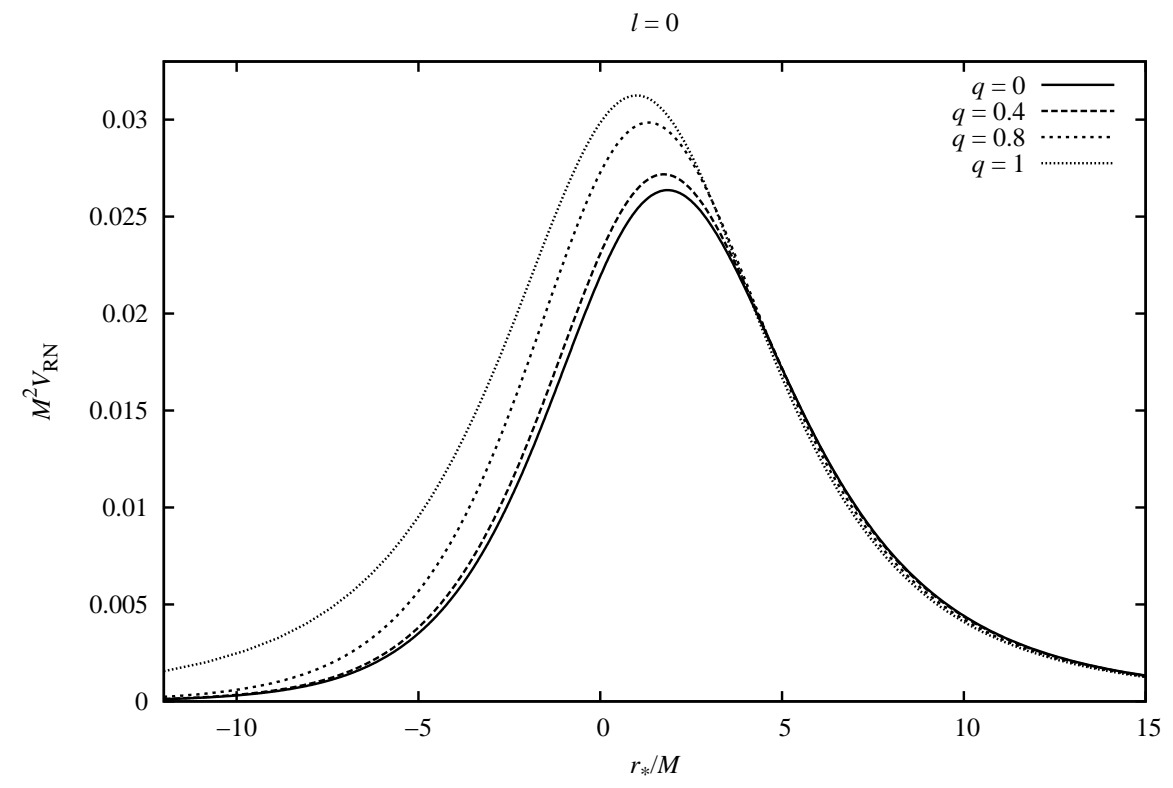

(b) As mesmas funções de (a) aqui em termos de $r_{*} / M$.

Figura 3.2: Em (a) podemos perceber que o máximo do potencial aumenta quando aumentamos $q$, ao mesmo tempo há um deslocamento do potencial para próximo do horizonte de eventos. Enquanto na Figura 3.1 o aumento do potencial está relacionado ao aumento do momento angular da onda, aqui fixamos $l$. 
As constantes que aparecem nas soluções (3.8), (3.9) e (3.10) determinam as características fundamentais das partes incidente, refletida e transmitida de cada onda parcial. Com estas constantes podemos escrever os coeficientes de reflexão e de transmissão, dados respectivamente por:

$$
\left|R_{\omega l}\right|^{2}=\left|\frac{A_{\omega l}^{\mathrm{ref}}}{A_{\omega l}^{\mathrm{in}}}\right|^{2}
$$

e

$$
\left|T_{\omega l}\right|^{2}=\left|\frac{A_{\omega l}^{\mathrm{tr}}}{A_{\omega l}^{\mathrm{in}}}\right|^{2},
$$

que obedecem à relação $\left|R_{\omega l}\right|^{2}+\left|T_{\omega l}\right|^{2}=1$. Além disso, a defasagem de cada onda parcial, que tem um papel fundamental na amplitude de espalhamento, é dada por [45]:

$$
\alpha_{l}(\omega)=\frac{1}{2 i} \ln \left[(-1)^{l+1} R_{\omega l}\right] .
$$

\subsection{Perturbações em buracos acústicos canônicos}

Como dito na seção 2.2, perturbações $(\delta \vec{v}=-\nabla \Phi)$ sobre o fluxo que gera buracos acústicos canônicos obedecem à equação de Klein-Gordon (1.1) sujeitas à métrica (2.14)-(2.15). Com base nisso, nosso objetivo nesta seção consiste em resolver a equação de Klein-Gordon. Prosseguimos de forma análoga à descrita na seção 3.1. Portanto, escrevendo

$$
\Phi=\left[\varphi_{\omega l}(r) / r\right] Y_{l m}(\theta, \phi) e^{-i \omega t},
$$

a função radial $\varphi_{\omega l}(r)$ é determinada pela seguinte equação:

$$
h \frac{d}{d r}\left[h \frac{d}{d r} \varphi_{\omega l}(r)\right]+\left[\omega^{2}-V_{\mathrm{c}}(r)\right] \varphi_{\omega l}(r)=0,
$$

onde

$$
V_{\mathrm{c}}(r)=\left(1-\frac{r_{\mathrm{c}}^{4}}{r^{4}}\right)\left[\frac{4 r_{\mathrm{c}}^{4}}{r^{6}}+\frac{l(l+1)}{r^{2}}\right] .
$$

Para tratar assintoticamente a função radial, lançamos mão da coordenada tartaruga, que no caso do buraco acústico canônico é

$$
x=r+\frac{r_{\mathrm{c}}}{4} \ln \left|\frac{r-r_{\mathrm{c}}}{r+r_{\mathrm{c}}}\right|-\frac{1}{2} r_{\mathrm{c}} \tan ^{-1}\left(\frac{r}{r_{\mathrm{c}}}\right)+\frac{\pi r_{\mathrm{c}}}{4} .
$$

Com esta coordenada a equação (3.14) fica

$$
\frac{d^{2}}{d x^{2}} \varphi_{\omega l}(x)+\left(\omega^{2}-V_{c}\right) \varphi_{\omega l}(x)=0
$$




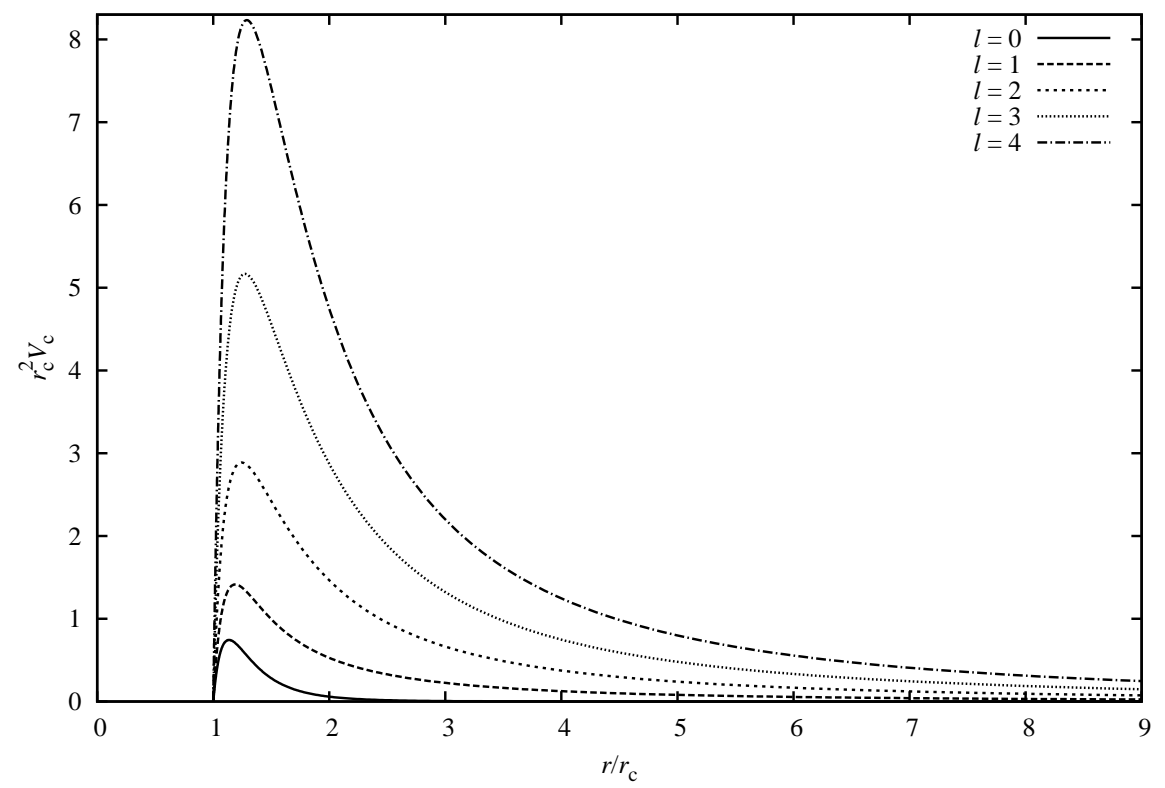

(a) Potencial efetivo para perturbações no fluxo que gera buracos acústicos canônicos. Aqui $l$ vai de 0 a 4 .

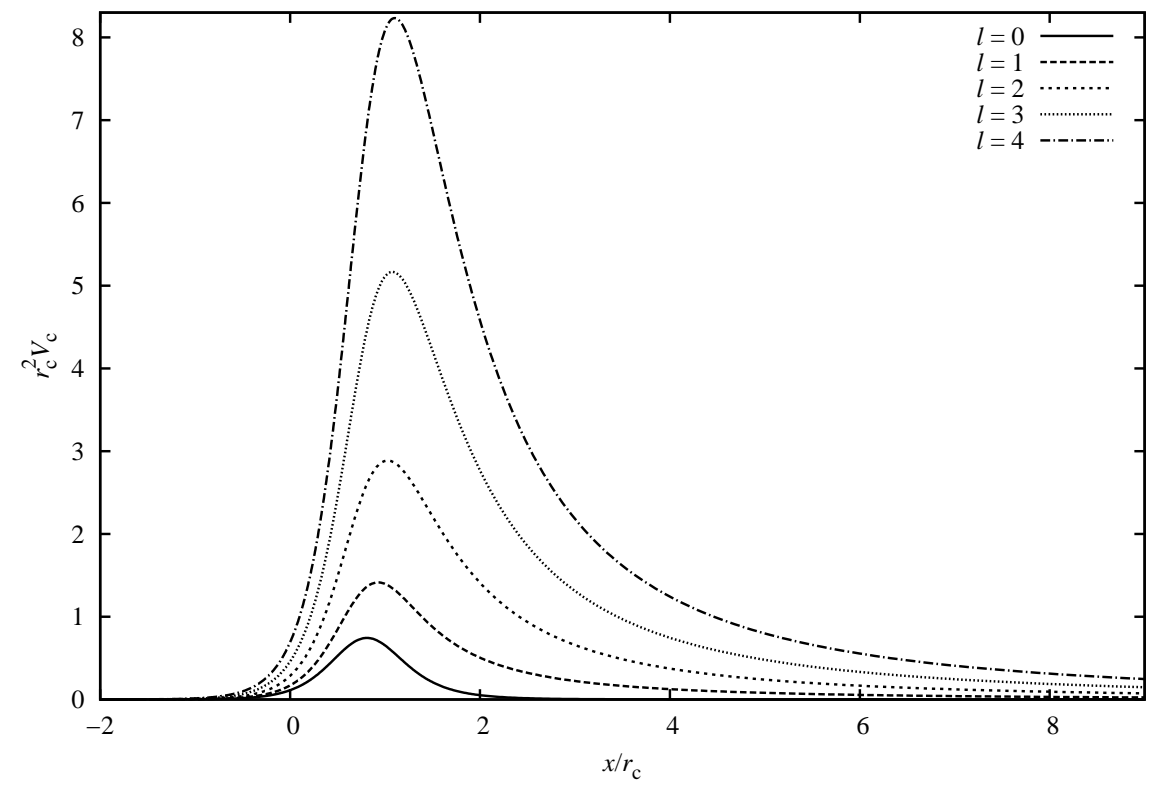

(b) $\mathrm{O}$ mesmo que em (a) aqui como função de $x / r_{\mathrm{c}}$.

Figura 3.3: Como no caso de buracos negros de Reissner-Nordström, o potencial efetivo aqui aumenta com $l$. Ele tende a zero no limite $r \rightarrow r_{\mathrm{c}}(x \rightarrow-\infty)$ e no infinito $(x \rightarrow+\infty)$. Quando $r \gg r_{\mathrm{c}}$, este potencial tende a $l(l+1) / r^{2}$. 
de modo que $V_{\mathrm{c}}$ tem papel de potencial efetivo no caso do som ao redor de buracos acústicos canônicos. Na Figura 3.3 plotamos este potencial efetivo. Basicamente, ele aumenta quando aumentamos o número quântico $l$ e vai a zero no horizonte de eventos e no infinito.

Observando o comportamento do potencial efetivo, é possível obter as soluções assintóticas para (3.17). Para $r \rightarrow r_{\mathrm{c}}(x \rightarrow-\infty)$, temos:

$$
\varphi_{\omega l}(x) \approx B_{\omega l}^{\mathrm{tr}} e^{-i \omega x}
$$

No caso de $r \gg r_{\mathrm{c}}$, o termo não desprezível no potencial é $l(l+1) / r^{2}$, de modo que:

$$
\varphi_{\omega l}(x) \approx \omega x\left[(-i)^{l+1} B_{\omega l}^{\mathrm{in}} h_{l}^{(1) *}(\omega x)+i^{l+1} B_{\omega l}^{\mathrm{ref}} h_{l}^{(1)}(\omega x)\right] .
$$

Quando $\omega x \gg l(l+1) / 2$, podemos ainda escrever:

$$
\psi_{\omega l}(x) \approx B_{\omega l}^{\mathrm{in}} e^{-i \omega x}+B_{\omega l}^{\mathrm{ref}} e^{i \omega x} .
$$

Como no caso de buracos negros de Reissner-Nordström, os coeficientes de reflexão e transmissão no caso de buracos acústicos canônicos são, respectivamente,

$$
\left|C_{\omega l}\right|^{2}=\left|\frac{B_{\omega l}^{\mathrm{ref}}}{B_{\omega l}^{\mathrm{in}}}\right|^{2}
$$

$\mathrm{e}$

$$
\left|D_{\omega l}\right|^{2}=\left|\frac{B_{\omega l}^{\mathrm{tr}}}{B_{\omega l}^{\mathrm{in}}}\right|^{2},
$$

que obedecem à relação $\left|C_{\omega l}\right|^{2}+\left|D_{\omega l}\right|^{2}=1$. A defasagem de cada onda parcial é neste caso

$$
\eta_{l}(\omega)=\frac{1}{2 i} \ln \left[(-1)^{l+1} C_{\omega l}\right]
$$

\subsection{O campo eletromagnético no espaço-tempo de Schwarzs- child}

Nesta seção analisamos o comportamento do campo eletromagnético ao redor de um buraco negro de Schwarzschild. Pudemos concluir que, no caso escalar, o problema se reduz a determinar as soluções das equações radiais. Para o campo eletromagnético, além disso, temos que encontrar condições de gauge que tornem a análise matemática do campo mais fácil. Neste sentido, lidaremos aqui com duas condições de gauge diferentes: o gauge de Coulomb esférico e o gauge de Feynman modificado [47]. Como veremos, o gauge de Coulomb esférico torna a análise do campo mais simples. Depois de obter estas soluções, por meio de uma transformação de gauge encontraremos a forma do campo no gauge de Feynman modificado. 


\subsubsection{Equações de Maxwell na geometria de Schwarzschild}

A densidade de lagrangeana para o campo eletromagnético livre é

$$
\mathcal{L}_{M}=-\frac{\sqrt{-g}}{4} F_{\mu \nu} F^{\mu \nu}
$$

sendo

$$
F_{\mu \nu}=\nabla_{\mu} A_{v}-\nabla_{v} A_{\mu}
$$

onde $A^{\mu}$ é o 4-potencial eletromagnético.

No espaço-tempo de Schwarzschild $R_{\mu \nu}=0$. Então, a partir da densidade de lagrangeana (3.24), encontramos que as equações de movimento de Euler-Lagrange para o vetor $A_{\mu}$ são

$$
\nabla_{\mu} \nabla^{\mu} A_{v}-\frac{\partial}{\partial x^{v}}\left(\nabla_{\mu} A^{\mu}\right)=0
$$

Na geometria de Schwarzschild os símbolos de Christoffel não nulos são

$$
\begin{array}{llrl}
\Gamma_{t r}^{t}=\Gamma_{r t}^{t}=\frac{1}{2} \frac{f^{\prime}}{f} ; & \Gamma_{t t}^{r}=\frac{1}{2} f f^{\prime} ; & \Gamma_{r r}^{r}=-\frac{1}{2} \frac{f^{\prime}}{f} ; \\
\Gamma_{\theta \theta}^{r}=-r f ; & \Gamma_{\phi \phi}^{r}=-r f \operatorname{sen}^{2} \theta ; & \Gamma_{r \theta}^{\theta}=\Gamma_{\theta r}^{\theta}=\frac{1}{r} ; \\
\Gamma_{\phi \phi}^{\theta}=-\operatorname{sen} \theta \cos \theta ; & \Gamma_{r \phi}^{\phi}=\Gamma_{\phi r}^{\phi}=\frac{1}{r} ; & \Gamma_{\theta \phi}^{\phi}=\Gamma_{\phi \theta}^{\phi}=\cot \theta,
\end{array}
$$

sendo que $f^{\prime}=d f / d r$ e com $f$ sendo dado por (2.2) com $Q=0$. (Nesta tese, sempre que estivermos abordando o campo eletromagnético, $f$ será relacionado à equação (2.2) com $Q=$ 0.)

Utilizando a métrica de Schwarzschild, implícita em 2.1) e 2.2) com $Q=0$, junto com as componentes não nulas da conexão, dadas em (3.27), a partir de (3.26) obtemos as seguintes equações para o 4-potencial eletromagnético:

$$
\begin{aligned}
& \square_{e} A_{t}-\frac{1}{f} \frac{\partial^{2} A_{t}}{\partial t^{2}}+f \frac{\partial^{2} A_{r}}{\partial t \partial r}-\frac{1}{r^{2}} \frac{\partial}{\partial t}\left(\tilde{\nabla}_{i} A^{i}\right)+f^{\prime} \frac{\partial A_{t}}{\partial r}+\frac{2 f}{r} \frac{\partial A_{r}}{\partial t}=0, \\
& \square_{e} A_{r}-\frac{1}{f} \frac{\partial^{2} A_{t}}{\partial r \partial t}+f \frac{\partial^{2} A_{r}}{\partial r^{2}}+\left(f^{\prime}+\frac{2 f}{r}\right) \frac{\partial A_{r}}{\partial r}-\frac{1}{r^{2}} \frac{\partial}{\partial r}\left(\tilde{\nabla}_{i} A^{i}\right)=0,
\end{aligned}
$$

$\mathrm{e}$

$$
\left(\frac{1}{f} \frac{\partial^{2}}{\partial t^{2}}-f \frac{\partial^{2}}{\partial r^{2}}-f^{\prime} \frac{\partial}{\partial r}\right) A_{i}-\frac{2 f}{r} \frac{\partial A_{r}}{\partial x^{i}}+\frac{1}{r^{2}} \tilde{\nabla}^{2} A_{i}+\frac{1}{r^{2}} A_{i}-\frac{\partial}{\partial x^{i}}\left(\nabla_{\mu} A^{\mu}\right)=0 .
$$

Nas equações acima, os índices latinos representam variáveis na esfera de raio unitário $S^{2}$ com signatura negativa e elemento de linha dado por $d l^{2}=-d \theta^{2}-\operatorname{sen}^{2} \theta d \phi^{2}$. Desta maneira temos:

$$
\tilde{\nabla}_{i} A^{i}=-\frac{\partial A_{\theta}}{\partial \theta}-\frac{1}{\operatorname{sen}^{2} \theta} \frac{\partial A_{\phi}}{\partial \phi}-\cot \theta A_{\theta}
$$




$$
\tilde{\nabla}^{2} A_{i}=\left\{\begin{array}{cc}
\tilde{\nabla}_{e}^{2} A_{\theta}+2 \cot \theta \frac{1}{\operatorname{sen}^{2} \theta} \frac{\partial A_{\phi}}{\partial \phi}+\cot ^{2} \theta A_{\theta} & \text { para } i=\theta \\
\tilde{\nabla}_{e}^{2} A_{\phi}-2 \cot \left(\frac{\partial A_{\theta}}{\partial \phi}-\frac{\partial A_{\phi}}{\partial \phi}\right)-A_{\phi} & \text { para } i=\phi
\end{array} .\right.
$$

Nas expressões acima, o índice " $e$ " é usado para representar os operadores escalares que são dados por:

$$
\square_{e}=\frac{1}{\sqrt{-g}} \frac{\partial}{\partial x^{\mu}}\left(\sqrt{-g} g^{\mu \nu} \frac{\partial}{\partial x^{v}}\right)
$$

e

$$
\tilde{\nabla}_{e}^{2}=\frac{1}{\sqrt{\tilde{g}}} \frac{\partial}{\partial x^{i}}\left(\sqrt{\tilde{g}} \tilde{g}^{i j} \frac{\partial}{\partial x^{j}}\right),
$$

com $\tilde{g}_{i j}$ sendo a métrica na superfície $S^{2}$. Podemos ainda escrever:

$$
\square_{e}=\frac{1}{f} \frac{\partial^{2}}{\partial t^{2}}-f \frac{\partial^{2}}{\partial r^{2}}-\left(f^{\prime}+\frac{2 f}{r}\right) \frac{\partial}{\partial r}+\frac{1}{r^{2}} \tilde{\nabla}_{e}^{2}
$$

$\mathrm{e}$

$$
\tilde{\nabla}_{e}^{2}=-\frac{\partial^{2}}{\partial \theta^{2}}-\cot \theta \frac{\partial}{\partial \theta}-\frac{1}{\operatorname{sen}^{2} \theta} \frac{\partial^{2}}{\partial \phi^{2}}
$$

Além disto, temos que

$$
\nabla_{\mu} A^{\mu}=\frac{1}{f} \frac{\partial A_{t}}{\partial t}-f \frac{\partial A_{r}}{\partial r}-\left(f^{\prime}+\frac{2 f}{r}\right) A_{r}+\frac{1}{r^{2}} \tilde{\nabla}_{i} A^{i}
$$

Com as equações acima temos tudo que necessitamos para encontrar o comportamento clássico do campo eletromagnético no espaço-tempo de Schwarzschild. Vale ressaltar que uma escolha de gauge adequada pode fazer com que dificuldades para encontrar soluções das equações de campo sejam amenizadas.

\subsubsection{Soluções no gauge de Coulomb esférico}

Nesta seção encontraremos as formas para o 4-potencial eletromagnético no gauge de Coulomb esférico. Como veremos, este gauge torna o tratamento das equações da seção anterior mais fácil

A condição para o gauge de Coulomb esférico é

$$
\tilde{\nabla}_{i} A^{i}=0
$$

Com esta condição, as equações para o 4-potencial eletromagnético 3.28, 3.29) e 3.30) se tornam

$$
\square_{e} A_{t}-\frac{1}{f} \frac{\partial^{2} A_{t}}{\partial t^{2}}+f \frac{\partial^{2} A_{r}}{\partial t \partial r}+f^{\prime} \frac{\partial A_{t}}{\partial r}+\frac{2 f}{r} \frac{\partial A_{r}}{\partial t}=0,
$$

\footnotetext{
${ }^{2}$ Uma prova de que esta condição de gauge é legítima pode ser encontrada em [48].
} 


$$
\square_{e} A_{r}-\frac{1}{f} \frac{\partial^{2} A_{t}}{\partial r \partial t}+f \frac{\partial^{2} A_{r}}{\partial r^{2}}+\left(f^{\prime}+\frac{2 f}{r}\right) \frac{\partial A_{r}}{\partial r}=0,
$$

$\mathrm{e}$

$$
\left(\frac{1}{f} \frac{\partial^{2}}{\partial t^{2}}-f \frac{\partial^{2}}{\partial r^{2}}-f^{\prime} \frac{\partial}{\partial r}\right) A_{i}+\frac{1}{r^{2}} \tilde{\nabla}^{2} A_{i}+\frac{1}{r^{2}} A_{i}-\frac{1}{f} \frac{\partial^{2} A_{t}}{\partial x^{i} \partial t}+f \frac{\partial^{2} A_{r}}{\partial x^{i} \partial r}+f^{\prime} \frac{\partial A_{r}}{\partial x^{i}}=0 .
$$

Para encontrar as soluções das equações acima, vamos agora considerar as diferentes polarizações do campo eletromagnético. Estas polarizações são dadas pelos modos de puro gauge, pelos modos físicos, com duas polarizações diferentes que simbolizaremos com o índice $\lambda=I$ e $I I$, e pelos modos não físicos. Neste trabalho, entretanto, só será necessária a abordagem dos modos físicos e o conhecimento da forma geral dos modos de puro gauge $\mathrm{H}^{3}$.

Os modos de puro gauge são modos que satisfazem a condição 3.35 e que podem ser escritos como:

$$
A_{\mu}^{(G)}=\nabla_{\mu} \Phi
$$

Desta definição, podemos concluir que $F_{\mu \nu}^{(G)}=\nabla_{\mu} A_{v}^{(G)}-\nabla_{v} A_{\mu}^{(G)}=0$. Neste caso, concluímos que os modos de puro gauge não contribuem com o processo de espalhamento. Apesar disto, como veremos no futuro, será útil determinar sua forma. Neste caso, da aplicação da condição de gauge (3.35) em 3.39), concluímos que

$$
\tilde{\nabla}_{e}^{2} \Phi=0
$$

Desta equação podemos concluir que $\Phi$ não possui dependência angular. Logo, os modos de puro gauge podem ser escritos como:

$$
A_{\mu}^{(G)}=\left(\partial_{t} \Phi(t, r), \partial_{r} \Phi(t, r), 0,0\right) .
$$

Devido ao fato de o espaço-tempo de Schwarzschild ser dotado de um campo de Killing global tipo tempo $\xi^{\mu}=\left(\partial_{t}\right)^{\mu}=(1,0,0,0)$, os modos físicos podem ser escritos da seguinte maneira:

$$
A_{\mu}^{(\lambda \omega l m)}=\zeta_{\mu}^{(\lambda \omega l m)} e^{-i \omega t}
$$

para $\omega>0$.

Para os modos físicos com $\lambda=I$ vamos escolher inicialmente

$$
A_{\mu}^{(I \omega l m)}=\left(A_{t}^{(I \omega l m)}, A_{r}^{(I \omega l m)}, 0,0\right) .
$$

Neste caso $A_{\mu}^{(I \omega l m)}$ satisfaz a condição de gauge 3.35 de maneira direta. Fazendo a separação de variáveis como $\zeta_{\mu}^{(I \omega l m)}=R_{\mu}^{(I \omega l)}(r) \Omega_{\mu}^{(l m)}(\theta, \phi)$, substituindo em 3.42 e o resultado em 3.38 ) concluímos que $\Omega_{t}^{(l m)}(\theta, \phi)=\Omega_{r}^{(l m)}(\theta, \phi)$ e que

$$
R_{t}^{(I \omega l)}(r)=\frac{i f}{\omega} \frac{d}{d r}\left[f R_{r}^{(I \omega l)}(r)\right] .
$$

\footnotetext{
${ }^{3}$ Os modos não físicos são aqueles que satisfazem as equações 3.36, 3.37) e 3.38 mas não satisfazem a condição (3.35) [48]. Estes modos são importantes na quantização do campo eletromagnético. No entanto, nesta tese não lidaremos com quantização, por isso não necessitamos analisar os modos não físicos.
} 
Com estes resultados e 3.37 obtemos que $\Omega_{t}^{(l m)}(\theta, \phi)=\Omega_{r}^{(l m)}(\theta, \phi)=Y_{l m}(\theta, \phi)$ e que

$$
f \frac{d}{d r}\left\{f \frac{d}{d r}\left[f R_{r}^{(I \omega l)}(r)\right]\right\}+\left[\omega^{2}-V_{\mathrm{e}}(r)\right] f R_{r}^{(I \omega l)}(r)=0,
$$

onde o potencial de espalhamento é

$$
V_{\mathrm{e}}=f \frac{l(l+1)}{r^{2}}
$$

que mostramos na Figura 3.4. Assim, temos

$$
A_{\mu}^{(I \omega l m)}=\left(\frac{i f}{\omega} \frac{d}{d r}\left[f R_{r}^{(I \omega l)}(r)\right], R_{r}^{(I \omega l)}(r), 0,0\right) Y_{l m}(\theta, \phi) e^{-i \omega t} .
$$

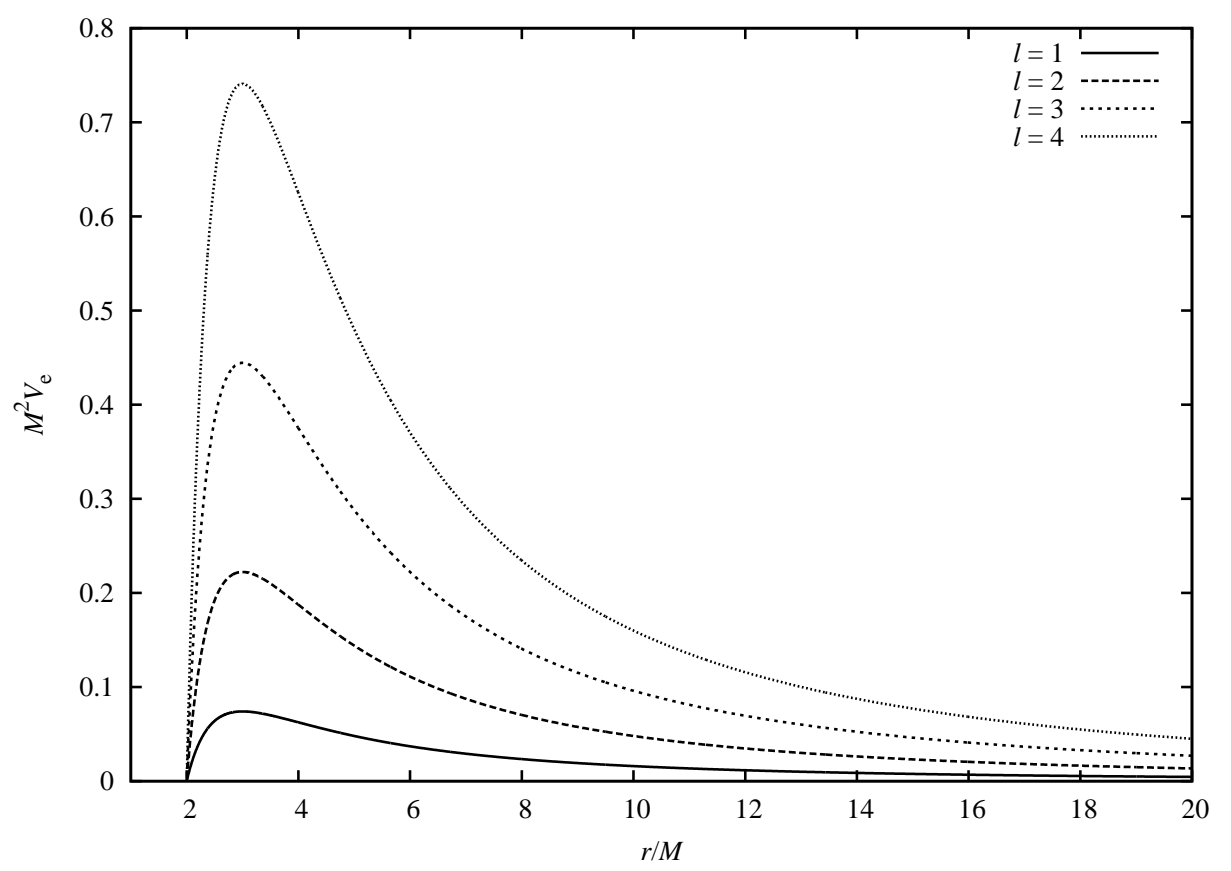

Figura 3.4: Potencial efetivo eletromagnético ao redor de buracos negros de Schwarzschild, dado pela equação (3.46), para alguns valores de $l$. Como em todos os casos abordados aqui, este potencial vai a zero no horizonte de eventos e no infinito. Além disso, o seu máximo aumenta com o aumento do valor de $l$.

Para $l=0,3.47$ pode ser escrita como:

$$
A_{\mu}^{(I \omega 00)}=\left(\frac{i f}{\omega} \frac{d}{d r}\left[f R_{r}^{(I \omega 0)}(r)\right], R_{r}^{(I \omega 0)}(r), 0,0\right) \frac{1}{\sqrt{4 \pi}} e^{-i \omega t} .
$$


Substituindo (3.48) em (3.36) obtemos que

$$
R_{r}^{(I \omega 0)}(r)=\frac{i}{\omega} \frac{d R_{t}^{(I \omega 0)(r)}}{d r}
$$

Desta maneira é possível escrever $A_{\mu}^{(I \omega 00)}=\nabla_{\mu} \Phi \operatorname{com} \Phi=\left(i / \sqrt{4 \pi \omega^{2}}\right) R_{t}^{(I \omega 0)}(r) e^{-i \omega t}$. Logo, para $l=0$ não existem modos $\operatorname{com} \lambda=I$ que não sejam de puro gauge e, por isso, trataremos de modos físicos $\operatorname{com} \lambda=I$ apenas para $l \geq 1$.

Para os modos $\operatorname{com} \lambda=I I$ vamos fazer

$$
A_{\mu}^{(I I \omega l m)}=\left(0,0, A_{\theta}^{(I I \omega l m)}, A_{\phi}^{(I I \omega l m)}\right) .
$$

Note que escolhemos $A_{\mu}^{(I \omega l m)}$ e $A_{\mu}^{(I I \omega l m)}$ separadamente de forma conveniente. Isto pode ser feito desde que estes modos satisfaçam as equações para o 4-potencial, as condições de gauge e que eles sejam ortogonais. Neste sentido, ao final desta seção provamos que $A_{\mu}^{I \omega l m}$ e $A_{\mu}^{I I \omega l m}$ escolhidos como (3.43) e (3.49) são ortogonais.

Os modos 3.49) devem satisfazer a condição de gauge (3.35). No entanto, sabemos que $\tilde{\nabla}^{i} Y_{i}^{l m}(\theta, \phi)=0, \operatorname{com} Y_{i}^{l m}(\theta, \phi)$ sendo os harmônicos esféricos vetoriais definidos da seguinte maneira [49]:

$$
Y_{i}^{l m}(\theta, \phi)=\frac{1}{\sqrt{l(l+1)}} \epsilon_{i j} \frac{\partial Y_{l m}(\theta, \phi)}{\partial x_{i}} \quad(l>0),
$$

onde $\epsilon_{\theta \phi}=-\epsilon_{\phi \theta}=\operatorname{sen} \theta$ e $\epsilon_{\theta \theta}=\epsilon_{\phi \phi}=0$. Com isto, concluímos que os modos $A_{\mu}^{(I I \omega l m)}$ possuem a seguite forma:

$$
A_{\mu}^{(I I \omega l m)}=R^{I I \omega l}(r)\left(0,0, Y_{\theta}^{l m}(\theta, \phi), Y_{\phi}^{l m}(\theta, \phi)\right) e^{-i \omega t} .
$$

Ressaltamos que os modos (3.51) são válidos apenas para $l>0$.

Substituindo 3.51) em 3.38) e usando

$$
\tilde{\nabla}^{2} Y_{i}^{l m}(\theta, \phi)=[l(l+1)-1] Y_{i}^{l m}(\theta, \phi)
$$

concluímos que

$$
f \frac{d}{d r}\left\{f \frac{d}{d r}\left[R^{(I I \omega l)}(r)\right]\right\}+\left[\omega^{2}-V_{\mathrm{e}}(r)\right] R^{(I I \omega l)}(r)=0,
$$

sendo que $V_{\mathrm{e}}(r)$ é dado em (3.46).

Notemos agora que $f R_{r}^{(I \omega l)}(r)$ e $R^{(I I \omega l)}(r)$ satisfazem a mesma equação. Portanto, definimos duas novas funções como $r \varphi_{\omega l}^{I}(r)=f R_{r}^{(I \omega l)}(r)$ e $r \varphi_{\omega l}^{I I}(r)=R^{(I I \omega l)}(r)$ de modo que as funções radiais de $A_{\mu}^{(I \omega l m)}$ e $A_{\mu}^{(I I \omega l m)}$ possam ser tratadas de maneira bastante semelhante. Sendo assim temos:

$$
A_{\mu}^{(I \omega l m)}=\left(\frac{i f}{\omega} \frac{d}{d r}\left[r \varphi_{\omega l}^{I}(r)\right], \frac{r \varphi_{\omega l}^{I}(r)}{f}, 0,0\right) Y_{l m}(\theta, \phi) e^{-i \omega t}
$$

$\mathrm{e}$

$$
A_{\mu}^{(I I \omega l m)}=r \varphi_{\omega l}^{I I}(r)\left(0,0, Y_{\theta}^{l m}(\theta, \phi), Y_{\phi}^{l m}(\theta, \phi)\right) e^{-i \omega t}
$$


$\operatorname{com} r \varphi_{\omega l}^{\lambda}(r)$ satisfazendo a seguinte equação:

$$
f \frac{d}{d r}\left\{f \frac{d}{d r}\left[r \varphi_{\omega l}^{\lambda}(r)\right]\right\}+\left[\omega^{2}-V_{\mathrm{e}}(r)\right] r \varphi_{\omega l}^{\lambda}=0 .
$$

Com o que obtemos até aqui já podemos encontrar as formas do 4-potencial no gauge de Feynman modificado. Entretanto, nos resta mostrar que realmente $A_{\mu}^{(I \omega l m)}$ e $A_{\mu}^{(I I \omega l m)}$ representam polarizações ortogonais. Para isto, utilizaremos o produto interno de Klein-Gordon generalizado, definido como [48]:

$$
\sigma_{K G}\left(A^{(i)}, A^{(j)}\right) \equiv \int_{\Sigma^{(3)}} d \Sigma^{(3)} n_{\mu} W^{\mu}\left[A^{(i)}, A^{(j)}\right]
$$

sendo $(i)$ usado para o conjunto de índices $(\lambda \omega l m)$. Aqui, $d \Sigma^{(3)}$ é o elemento invariante do 3volume da superfície de Cauchy $\Sigma$ e $n^{\mu}$ é o vetor unitário ortogonal a $\Sigma$ apontando para o futuro e dado por $n^{\mu}=f^{-1 / 2} \delta_{0}^{\mu}$. Além disto, temos a corrente conservada definida como:

$$
W^{\mu}\left[A^{(i)}, A^{(j)}\right] \equiv i\left[A_{v}^{(i) *} \Pi^{(j) \mu v}-\Pi^{i \mu v *} A_{v}^{(j)}\right]
$$

sendo que $\Pi^{(j) \mu v}$ é o momento conjugado associado aos modos $A_{\mu}^{(j)}$, definido como:

$$
\left.\Pi^{(j) \mu v} \equiv \frac{1}{\sqrt{-g}} \frac{\partial \mathcal{L}}{\partial\left(\nabla_{\mu} A_{\nu}\right)}\right|_{A_{\mu}=A_{\mu}^{(j)}}
$$

Utilizando (3.24) em (3.59) obtemos que

$$
\Pi^{(j) \mu v}=-\left[F^{\mu v}\right]_{A_{\mu}=A_{\mu}^{(j)}} .
$$

Substituindo 3.60 em 3.58, o resultado em 3.57 quando $A_{\mu}^{(i)}=A_{\mu}^{(I \omega l m)}$ e $A_{\mu}^{(j)}=A_{\mu}^{(I I \omega l m)}$, com os modos dados em 3.43) e 3.49, e usando que $\nabla^{\mu} A_{\mu}^{(I I \omega l m)}=0$, obtemos

$$
\sigma_{K G}\left(A^{(I \omega l m)}, A^{(I I \omega l m)}\right)=\int_{\Sigma^{(3)}} d \Sigma^{(3)} n_{\mu} \nabla_{\nu} U^{\nu \mu}
$$

sendo $U^{v \mu}=A^{(I \omega l m) \nu *} A^{(I I \omega l m) \mu}-A^{(I I \omega l m) v} A^{(I \omega l m) \mu *}$. Utilizando o teorema de Gauss, concluímos que $\sigma_{K G}\left(A^{(I \omega l m)}, A^{(I I \omega l m)}\right)=0$. Logo, $A_{\mu}^{(I \omega l m)}$ e $A_{\mu}^{(I I \omega l m)}$ são ortogonais.

É possível provar que o produto interno de Klein-Gordon generalizado é invariante sob transformações de gauge [48]. Sendo assim, quaisquer modos obtidos a partir de $A_{\mu}^{(I \omega l m)} \mathrm{e}$ $A_{\mu}^{(I I \omega l m)}$ por uma transformação de gauge devem ser também ortogonais. 


\subsubsection{Soluções no gauge de Feynman modificado}

Para a análise do espalhamento é melhor que abordemos o 4-potencial eletromagnético no gauge de Feynman modificado. Para isto, partiremos da estrutura do 4-potencial obtido na subseção anterior. Isto é feito através de uma transformação de gauge dada por

$$
A_{\mu}^{(i)} \rightarrow A_{\mu}^{\prime(i)}=A_{\mu}^{(i)}-\nabla_{\mu} \Psi^{(i)},
$$

sendo $A_{\mu}^{\prime(i)}$ a representação do 4-potencial no novo gauge. Usando a transformação acima, 3.43) e 3.49, podemos escrever o 4-potencial no gauge de Feynman modificado da seguinte forma:

$$
A_{\mu}^{(I \omega l m)}=\left(A_{t}^{(I \omega l m)}-\partial_{t} \Psi^{I}, A_{r}^{(I \omega l m)}-\partial_{r} \Psi^{I},-\partial_{\theta} \Psi^{I},-\partial_{\phi} \Psi^{I}\right)
$$

e

$$
A_{\mu}^{\prime(I I \omega l m)}=\left(-\partial_{t} \Psi^{I I},-\partial_{r} \Psi^{I I}, A_{\theta}^{(I I \omega l m)}-\partial_{\theta} \Psi^{I I}, A_{\phi}^{(I I \omega l m)}-\partial_{\phi} \Psi^{I I}\right) .
$$

A condição para o gauge de Feynman modificado é

$$
\nabla_{\mu} A^{\prime(i) \mu}+K_{\mu} A^{\prime(i) \mu}=0
$$

onde $K^{\mu}=\left(0, f^{\prime}, 0,0\right)$. Aplicando a condição 3.65 em 3.63 , encontramos que $\Psi^{I}$ deve satisfazer a seguinte equação:

$$
-\frac{1}{f} \frac{\partial^{2} \Psi^{I}}{\partial t^{2}}+f \frac{\partial^{2} \Psi^{I}}{\partial r^{2}}+\frac{2 f}{r} \frac{\partial \Psi^{I}}{\partial r}-\frac{1}{r^{2}} \tilde{\nabla}_{e}^{2} \Psi^{I}+\left(\frac{r f^{\prime}}{f}-2\right) \varphi_{\omega l}^{I}(r) Y_{l m}(\theta, \phi) e^{-i \omega t}=0 .
$$

Podemos separar as variáveis desta equação fazendo $\Psi^{I}=q(r) Y_{l m}(\theta, \phi) e^{-i \omega t}$. Com isto obtemos

$$
f \frac{d^{2} q(r)}{d r^{2}}+\frac{2 f}{r} \frac{d q(r)}{d r}+\left[\omega^{2}-V_{\mathrm{e}}(r)\right] \frac{q(r)}{f}+\left(\frac{r f^{\prime}}{f}-2\right) \varphi_{\omega l}^{I}(r)=0,
$$

de onde concluímos que

$$
\Psi^{I}=-\frac{f}{\omega^{2}} \frac{d}{d r}\left[r \varphi_{\omega l}^{I}(r)\right] Y_{l m}(\theta, \phi) e^{-i \omega t} .
$$

Substituindo 3.68 em 3.63 concluímos que os modos $A_{\mu}^{\prime(I \omega l m)}$ são dados por

$$
\begin{aligned}
A_{\mu}^{\prime(I \omega l m)}= & \frac{1}{\omega^{2}}\left(0, l(l+1) \frac{\varphi_{\omega l}^{I}(r)}{r} Y_{l m}(\theta, \phi), f \frac{d}{d r}\left[r \varphi_{\omega l}^{I}(r)\right] \partial_{\theta} Y_{l m}(\theta, \phi),\right. \\
& \left.f \frac{d}{d r}\left[r \varphi_{\omega l}^{I}(r)\right] \partial_{\phi} Y_{l m}(\theta, \phi)\right) e^{-i \omega t} .
\end{aligned}
$$

Para $\Psi^{I I}$ a equação que obtemos é

$$
-\frac{1}{f} \frac{\partial^{2} \Psi^{I I}}{\partial t^{2}}+f \frac{\partial^{2} \Psi^{I I}}{\partial r^{2}}+\frac{2 f}{r} \frac{\partial \Psi^{I I}}{\partial r}-\frac{1}{r^{2}} \tilde{\nabla}_{e}^{2} \Psi^{I I}=0
$$


Notando que $\Psi^{I I}=0$ satisfaz trivialmente 3.70 , concluímos que $A_{\mu}^{\prime(I I \omega l m)}=A_{\mu}^{(I I \omega l m)}$. Assim, a forma de $A_{\mu}^{(I I \omega l m)}$ é

$$
A_{\mu}^{\prime(I I \omega l m)}=\left(0,0, r \varphi_{\omega l}^{I I}(r) Y_{\theta}^{l m}(\theta, \phi), r \varphi_{\omega l}^{I I}(r) Y_{\phi}^{l m}(\theta, \phi)\right) e^{-i \omega t} .
$$

Com 3.69 e 3.71 nos basta encontrar a forma da função radial $\varphi_{\omega l}^{\lambda}(r)$ dada pela solução da equação (3.56) para termos a forma completa do 4-potencial.

É importante notar, por meio das equações 3.69 e (3.71), que para $l=0$ não existem modos físicos.

\subsubsection{Soluções assintóticas}

Vamos agora encontrar soluções analíticas para a equação radial (3.56). Para pontos próximos ao horizonte eventos do buraco negro, usamos a coordenada de Wheeler, que é dada por (3.5). Com esta coordenada a equação (3.56) é escrita como:

$$
\frac{d^{2}}{d r_{*}^{2}}\left[r \varphi_{\omega l}^{\lambda}(r)\right]+\left[\omega^{2}-V_{\mathrm{e}}(r)\right] r \varphi_{\omega l}^{\lambda}(r)=0
$$

Na figura 3.5 plotamos o potencial efetivo $V_{\mathrm{e}}$, dado por 3.46 , em termos da coordenada de Wheeler. Lá vemos que ele vai a zero quando $r_{*} \rightarrow \pm \infty$, de modo que a forma assintótica de (3.72) pode ser facilmente determinada.

Para pontos extremamente próximos ao horizonte de eventos do buraco negro, $r \approx r_{\mathrm{S}}, \mathrm{o}$ potencial efetivo vai a zero, como é mostrado na figura 3.4. Neste caso, a forma de $\varphi_{\omega l}^{\lambda}$ para modos provenientes do infinito, quando muito próximos ao horizonte de eventos do buraco negro, é

$$
r \varphi_{\omega l}^{\lambda}\left(r_{*}\right) \approx B_{\omega l}^{\lambda \mathrm{tr}} e^{-i \omega r_{*}}
$$

Para $r \gg r_{\mathrm{S}}$, a forma de $r \varphi_{\omega l}^{\lambda}\left(r_{*}\right)$ é a mesma que a parte radial dos modos do campo escalar não massivo:

$$
r \varphi_{\omega l}^{\lambda}\left(r_{*}\right) \approx \omega r_{*}\left[(-i)^{l+1} B_{\omega l}^{\lambda \mathrm{in}} h_{l}^{(1) *}\left(\omega r_{*}\right)+i^{l+1} B_{\omega l}^{\lambda \mathrm{ref}} h_{l}^{(1)}\left(\omega r_{*}\right)\right]
$$

No caso em que $\omega r_{*} \gg l(l+1) / 2$, usando a forma assintótica da função de Hankel esférica obtemos que

$$
r \varphi_{\omega l}^{\lambda}\left(r_{*}\right) \approx B_{\omega l}^{\lambda \mathrm{in}} e^{-i \omega r_{*}}+B_{\omega l}^{\lambda \mathrm{ref}} e^{i \omega r_{*}} .
$$

Ainda como nos casos escalares, podemos definir os coeficientes de reflexão e transmissão a partir das soluções assintóticas. Assim temos que

$$
\left|T_{\omega l}^{\lambda}\right|^{2}=\left|\frac{B_{\omega l}^{\lambda \mathrm{tr}}}{B_{\omega l}^{\lambda \mathrm{in}}}\right|^{2}
$$

é o coeficiente de transmissão e

$$
\left|R_{\omega l}^{\lambda}\right|^{2}=\left|\frac{B_{\omega l}^{\lambda \mathrm{ref}}}{B_{\omega l}^{\lambda \mathrm{in}}}\right|^{2}
$$




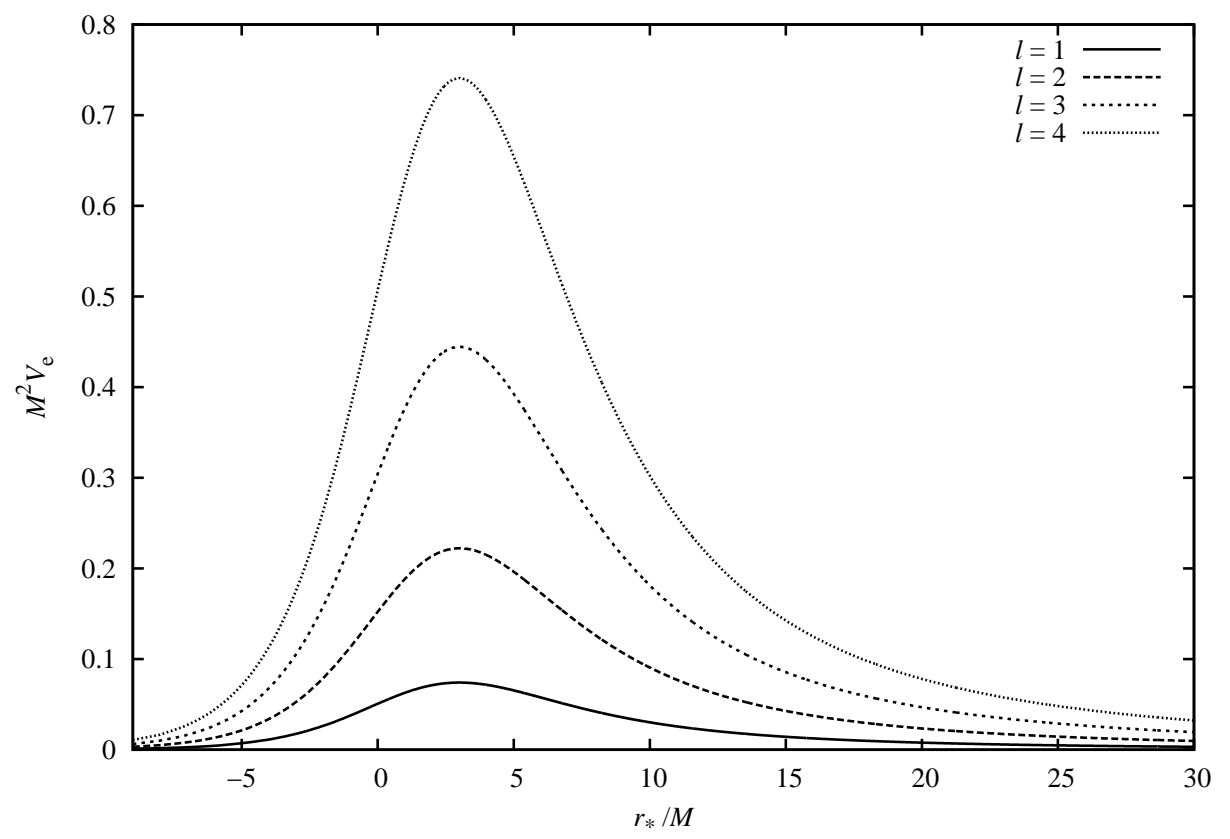

Figura 3.5: Potencial efetivo eletromagnético em Schwarzschild plotado em termos da coordenada de Wheeler. Aqui vemos que este potencial vai a zero quando $r_{*} \rightarrow-\infty\left(r \approx r_{\mathrm{S}}\right) \mathrm{e}$ $r_{*} \rightarrow \infty(r \rightarrow \infty)$. Essa percepção do comportamento do potencial facilita a determinação da parte radial dos modos eletromagnéticos nestes limites.

é o coeficiente de reflexão. Os coeficientes de reflexão e transmissão satisfazem $\left|R_{\omega l}^{\lambda}\right|^{2}+\left|T_{\omega l}^{\lambda}\right|^{2}=$ 1. Definimos também a defasagem do campo eletromagnético como:

$$
\delta_{l}^{\lambda}(\omega)=\frac{1}{2 i} \ln \left[(-1)^{l+1} R_{\omega l}^{\lambda}\right] .
$$




\section{Capítulo 4}

\section{Seção de choque}

O presente capítulo traz as definições das seções de choque de absorção, espalhamento e total e análises das possíveis aproximações das mesmas (com exceção da seção de choque de absorção em altas frequências, já abordada no Capítulo 2). As definições de seções de choque para buracos negros são basicamente as mesmas encontradas em livros textos de Mecânica Quântica, como por exemplo nas referências [45] e [50]. Quanto às aproximações, por se tratarem de buracos negros, é possível encontrar algumas em artigos científicos, como é o caso da seção de choque de espalhamento no limite de ângulos pequenos para Reissner-Nordström [51, 52, 53]. No caso de buracos acústicos, entretanto, por estarem no contexto de uma área da física que é relativamente nova, pouca coisa pode ser encontrada na literatura. Sendo assim, na medida do possível, apresentaremos os detalhes das aproximações. Ainda neste capítulo falamos da aproximação para o efeito glória, muito importante para a análise e comparação dos resultados da seção de choque diferencial de espalhamento.

\subsection{Definições}

Podemos definir a seção de choque diferencial de espalhamento da seguinte maneira [50]:

$$
d \sigma_{\mathrm{el}} \equiv \frac{\text { Número de partículas espalhadas por unidade de tempo no ângulo sólido } d \Omega}{\text { Fluxo de partículas incidentes }} .
$$

(O subescrito "el" refere-se à parte elástica do espalhamento [45, 50].) No caso do campo escalar, esta definição pode ser traduzida na seguinte fórmula:

$$
\frac{d \sigma_{\mathrm{el}}}{d \Omega}=\left|f_{\omega}(\theta)\right|^{2}
$$

na qual $f_{\omega}(\theta)$ é a amplitude de espalhamento dada por:

$$
f_{\omega}(\theta)=\frac{1}{2 i \omega} \sum_{l=0}^{\infty}(2 l+1)\left[e^{2 i \delta_{l}(\omega)}-1\right] P_{l}(\cos \theta),
$$


$\operatorname{com} \delta_{l}(\omega)$ sendo a defasagem de cada onda parcial e $P_{l}(\cos \theta)$ sendo os polinômios de Legendre [46]. [No caso de buracos negros de Reissner-Nordström devemos substituir $\delta_{l}(\omega)$ por (3.13) e no caso de buracos acústicos canônicos por (3.23).]

No caso do campo eletromagnético, em princípio, cada polarização deve ter um comportamento distinto no processo de espalhamento. No entanto, devido ao espaço tempo de Schwarzschild se esfericamente simétrico, as defasagens das duas diferentes polarizações são iguais. Nesse sentido, a seção de choque de espalhamento eletromagnética é dada por [54, 55]:

$$
\frac{d \sigma_{\mathrm{el}}^{\mathrm{e}}}{d \Omega}=\frac{1}{4 \omega^{2}}\left|\sum_{l=1}^{\infty} \frac{2 l+1}{l(l+1)} e^{2 i \delta_{l}^{\lambda}(\omega)}\left[\frac{P_{l}^{1}(\cos \theta)}{\operatorname{sen} \theta}+\frac{d}{d \theta} P_{l}^{1}(\cos \theta)\right]\right|^{2},
$$

em que $P_{l}^{m}(\cos \theta)$ são as funções de Legendre associadas.

Como veremos aqui, a seção de choque diferencial de espalhamento de buracos acústicos canônicos é finita para qualquer valor do ângulo de espalhamento. Nesse caso, portanto, podemos calcular a seção de choque de espalhamento. Para o campo escalar não massivo, ela é obtida através da integração de 4.2 no ângulo sólido resultando em:

$$
\sigma_{\mathrm{el}}=\sum_{l=0}^{\infty} \sigma_{\mathrm{el}}^{(l)}=\frac{\pi}{\omega^{2}} \sum_{l=0}^{\infty}(2 l+1)\left|1-e^{2 i \delta_{l}(\omega)}\right|^{2},
$$

na qual $\sigma_{\text {el }}^{(l)}$ é a seção de choque de espalhamento de cada onda parcial. A seção de choque diferencial de espalhamento de buracos negros de Schwarzschild e Reissner-Nordström divergem para $\theta \rightarrow 0$, de modo que a sua integração no ângulo sólido é infinita.

A seção de choque de absorção é definida como:

$$
\sigma_{\text {abs }} \equiv \frac{\text { Número de partículas absorvidas por unidade de tempo }}{\text { Fluxo de partículas incidentes }} .
$$

A partir desta definição, podemos mostrar que:

$$
\sigma_{\mathrm{abs}}=\sum_{l=s}^{\infty} \sigma_{\mathrm{abs}}^{(l)}=\frac{\pi}{\omega^{2}} \sum_{l=s}^{\infty}(2 l+1)\left(1-\left|e^{2 i \delta_{l}(\omega)}\right|^{2}\right),
$$

na qual $\sigma_{\text {abs }}^{(l)}$ representa a seção de choque de absorção de cada onda parcial e $s=0$ ou 1 dependendo se estamos abordando o campo escalar ou eletromagnético, respectivamente. Notemos ainda que $\delta_{l}(\omega)$ representa (3.13) no caso de Reissner-Nordström, (3.23) no caso de buracos acústicos canônicos e 3.78) no caso do espalhamento eletromagnético em Schwarzschild. Neste último caso, cada polarização deveria, em princípio, contribuir de forma diferente para a seção de choque de absorção. Porém, como dito acima, as defasagens das duas polarizações são iguais, de forma que elas acabam contribuindo de mesma forma, tanto para a seção de choque de absorção, quanto de espalhamento.

Existe ainda a seção de choque total que é a soma da seção de choque de absorção com a seção de choque de espalhamento. É evidente então que a ela é infinita no caso de buracos negros de Schwarzschild e Reissner-Nordström. Porém, ela é finita no caso de buracos acústicos 
canônicos, de forma que a apresentaremos para este caso. Portanto, no caso escalar, somando (4.5) e (4.7), obtemos:

$$
\sigma_{\text {tot }}=\frac{2 \pi}{\omega^{2}} \sum_{l=0}^{\infty}(2 l+1)\left(1-\operatorname{Re}\left\{e^{2 i \delta_{l}(\omega)}\right\}\right) .
$$

É possível ainda escrever a seção de choque total em termos da amplitude de espalhamento (4.3). Nesse caso, temos:

$$
\sigma_{\text {tot }}=\frac{4 \pi}{\omega} \operatorname{Im}\left\{f_{\omega}(0)\right\}
$$

que constitui o teorema ótico [45, 50].

\subsection{Seção de choque de absorção em baixas frequências}

A seção de choque de absorção em baixas frequências pode ser obtida por meio de aproximações das equações radiais (3.2), (3.14) e (3.56). Para buracos negros de Reissner-Nordström, consideramos cada caso de intensidade de carga separadamente. Aqui mostramos a obtenção da seção de choque de absorção em baixas frequências somente para o caso de Schwarzschild e de buracos negros de Reissner-Nordström extremos.

Exitem trabalhos que tratam a seção de choque de absorção do campo escalar não massivo de forma generalizada. Por exemplo, em [56], a seção de choque de absorção em baixas frequências é calculada para buracos negros esfericamente simétricos. Lá é mostrado que, sendo o espaço-tempo do exterior do buraco negro estacionário e assintoticamente plano, a seção de choque de absorção do campo escalar não massivo em baixas frequências se iguala à área do buraco negro, independentemente do número de dimensões.

Outra pesquisa do gênero foi desenvolvida por A. Higuchi em [57]. Nesse artigo ele mostrou que a universalidade da seção de choque de absorção para o campo escalar não massivo em baixas frequências, mostrada por Das et al em [56], ainda se mantém para buracos negros que não sejam esfericamente simétricos, porém desde que sejam estacionários. Nesse caso, mesmo um buraco negro de Kerr-Newman (buraco negro com carga e rotação [5, 38]) teria a seção de choque de absorção para o campo escalar não massivo em baixas frequências igual à sua área.

\subsubsection{Caso escalar em Schwarzschild}

No caso de buracos negros de Schwarzschild, a solução da equação (3.2) quando $\omega=0$ é

$$
\psi_{0 l}(r)=a_{1} r P_{l}(z)+a_{2} r Q_{l}(z)
$$

na qual $a_{1}$ e $a_{2}$ são constantes, $P_{l}(z)$ e $Q_{l}(z)$ são funções de Legendre de primeiro e segundo tipo [46], respectivamente, e

$$
z=\frac{r}{M}-1
$$


Em se tratando de espalhamento, devemos esperar que as ondas no limite $\omega \rightarrow 0$ devam ser finitas no horizonte de eventos, uma vez que quase toda a onda deva ser espalhada pelo potencial efetivo (3.3). Nesse caso, devemos ter $a_{2}=0$, já que $Q_{l}(z) \rightarrow \infty$ se $z \rightarrow 1$ (que é quando $r \rightarrow r_{\mathrm{S}}$ ).

Para determinarmos $a_{1}$, devemos reescrever (4.10) no limite $r \gg r_{\mathrm{S}}$ e compará-la com (3.9), tomando esta última no limite $\omega r \ll 1$. Nesse limite, (3.9) fica:

$$
\psi_{\omega l} \approx\left[(-i)^{l+1} A_{\omega l}^{\mathrm{in}}+i^{l+1} A_{\omega l}^{\mathrm{ref}}\right] \frac{2^{l} l !}{(2 l+1) !}\left(\omega r_{*}\right)^{l+1}+i\left[(-i)^{l+1} A_{\omega l}^{\mathrm{in}}-i^{l+1} A_{\omega l}^{\mathrm{ref}}\right] \frac{(2 l) !}{2^{l} l !}\left(\omega r_{*}\right)^{-l}
$$

onde usamos que $h_{l}^{(1)}(x)=j_{l}(x)+i n_{l}(x), \operatorname{com} j_{l}(x)$ e $n_{l}(x)$ sendo as funções de Bessel e de Neumann esféricas, respectivamente. Além disso, aproximamos estas funções por

$$
j_{l}(x) \approx \frac{2^{l} l !}{(2 l+1) !} x^{l}
$$

$\mathrm{e}$

$$
n_{l}(x) \approx-\frac{(2 l) !}{2^{l} l !} x^{-l-1},
$$

para $x \ll 1$ [46].

Mais uma vez temos que considerar que a solução em baixas frequências não seja divergente. Nesse caso, o termo proporcional a $\left(\omega r_{*}\right)^{-l}$ na expressão 4.12 deva ser muito menor que o termo proporcional a $\left(\omega r_{*}\right)^{l+1}$. Isso é possível se $A_{\omega l}^{\mathrm{ref}} \approx(-1)^{l+1} A_{\omega l}^{\mathrm{in}}-O(\omega)$, ou seja, desde que a onda seja quase que completamente espalhada. Isso é esperado no limite de baixas frequências. Sendo assim 4.12 se torna:

$$
\psi_{\omega l} \approx(-i)^{l+1} A_{\omega l}^{\text {in }} \frac{2^{l+1} l !}{(2 l+1) !}(\omega r)^{l+1},
$$

onde consideramos ainda que $\omega r_{*} \approx \omega r$ no limite de baixas frequências quando $r \gg r_{\mathrm{S}}$.

Agora, tomemos a solução em que $\omega=0$ assintoticamente. Nesse caso, usando que

$$
P_{l}(z) \approx \frac{(2 l) !}{2^{l}(l !)^{2}} z^{l},
$$

concluímos que

$$
\psi_{0 l} \approx a_{1} \frac{(2 l) !}{2^{l}(l !)^{2}} \frac{r^{l+1}}{M^{l}} .
$$

A solução em baixas frequências é obtida quando comparamos (4.15) com (4.16). Nesse caso, a dependência com a frequência está contida na constante $a_{1}$ que, da comparação destas equações, é

$$
a_{1}=(-i)^{l+1} A_{\omega l}^{\text {in }} \frac{2^{2 l+1}(l !)^{3}}{(2 l) !(2 l+1) !} M^{l} \omega^{l+1} \text {, }
$$


e consequentemente a solução em baixas frequências será

$$
\psi_{\omega l}(r) \approx(-i)^{l+1} A_{\omega l}^{\text {in }} \frac{2^{2 l+1}(l !)^{3}}{(2 l) !(2 l+1) !} M^{l} \omega^{l+1} r P_{l}(z)
$$

A seção de choque de absorção em baixas frequências é obtida quando comparamos (4.18) no limite $r \rightarrow r_{\mathrm{S}}$ com (3.8) quando $\left|\omega r_{*}\right| \ll 1$. Para (4.18), no limite em que $r \rightarrow r_{\mathrm{S}}(z \rightarrow 1)$, lembrando que $P_{l}(1)=1$, concluímos que

$$
\psi_{\omega l} \approx(-i)^{l+1} A_{\omega l}^{\text {in }} \frac{2^{2 l+2}(l !)^{3}}{(2 l) !(2 l+1) !}(M \omega)^{l+1} .
$$

Quando $\left|\omega r_{*}\right| \ll 1,3.8$ se torna

$$
\psi_{\omega l} \approx A_{\omega l}^{\mathrm{tr}} .
$$

As expressões (4.19) e (4.20) descrevem ondas com baixas frequências próximas ao horizonte de eventos do buraco negro e, portanto, são iguais. Considerando essa igualdade, obtemos que

$$
T_{\omega l}=\frac{A_{\omega l}^{\mathrm{tr}}}{A_{\omega l}^{\mathrm{in}}} \approx(-i)^{l+1} \frac{2^{2 l+2}(l !)^{3}}{(2 l) !(2 l+1) !}(M \omega)^{l+1}
$$

no limite de baixas frequências. Lembrando que $\left|R_{\omega l}\right|^{2}+\left|T_{\omega l}\right|^{2}=1$ e usando 3.13 e (4.7), concluímos que a seção de choque de absorção em baixas frequências para campo escalar não massivo em Schwarzschild é

$$
\begin{aligned}
\sigma_{\mathrm{abs}}^{\mathrm{S}} & =\frac{\pi}{\omega^{2}} \sum_{l=0}^{\infty}(2 l+1)\left|T_{\omega l}\right|^{2} \\
& \approx \pi M^{2} \sum_{l=0}^{\infty}(2 l+1)\left[\frac{2^{2 l+2}(l !)^{3}}{(2 l) !(2 l+1) !}\right]^{2}(M \omega)^{2 l} .
\end{aligned}
$$

Analisando e expressão 4.22, concluímos que as contribuições parciais para a seção de choque de absorção vão a zero quando $l \geq 1$, já que $M \omega \ll 1$. Portanto, em concordância com o demonstrado em [56], obtemos que a contribuição não desprezível para a seção de choque de absorção em baixas frequências vem da onda parcial em que $l=0$. Sendo assim, temos

$$
\begin{aligned}
\sigma_{\mathrm{abs}}^{\mathrm{S}} & \approx \sigma_{\mathrm{abs}}^{\mathrm{S}(0)} \\
& \approx 16 \pi M^{2}=4 \pi r_{\mathrm{S}}^{2}=A_{\mathrm{S}} .
\end{aligned}
$$

Portanto, como esperado de [56] e [57], a seção de choque de absorção do campo escalar não massivo em baixas frequências para buracos negros de Schwarzschild é igual à área do buraco negro, $A_{\mathrm{S}}$. 


\subsubsection{Caso escalar em Reissner-Nordström extremo}

O procedimento para o cálculo da seção de choque de absorção em baixas frequências de buracos negros de Reissner-Nordström extremos é bastante similar ao caso de buracos negros de Schwarzschild. No entanto, existe uma diferença no que se refere ao tratamento da solução da equação (3.7) quando estamos próximos ao horizonte de eventos. Isso acontece porque, como pode ser observado na Figura 3.2(b), o potencial efetivo de buracos negros extremos cai mais lentamente a zero do que para outros valores de $q$ quanto $r_{*} \rightarrow-\infty$. Portanto, no caso de buracos negros de Reissner-Nordström extremos, temos que analisar mais cuidadosamente a solução próxima ao horizonte.

A coordenada $r_{*}$ é dada por (3.6) no caso de buracos negros de Reissner-Nordström extremos. No limite $r \rightarrow M$, considerando apenas o maior termo, quando esta coordenada pode ser aproximada por

$$
r_{*} \approx-\frac{M^{2}}{r-M} .
$$

Nesse limite, o potencial efetivo 3.3 pode ser descrito por

$$
V_{\mathrm{RN}} \approx \frac{l(l+1)}{r_{*}^{2}}
$$

e, consequentemente, a solução para a equação (3.7) é

$$
\psi_{\omega l}\left(r_{*}\right) \approx i^{l+1} A_{\omega l}^{\mathrm{tr}} \sqrt{-\frac{\pi \omega r_{*}}{2}} H_{l+1 / 2}^{(1)}\left(-\omega r_{*}\right)
$$

sendo $H_{l}^{(1)}(x)$ a função de Hankel. Notemos que no limite $\left|\omega r_{*}\right| \gg l(l+1) / 2$, esta expressão se reduz a (3.8). No limite de baixas frequências, ou seja, $\left|\omega r_{*}\right| \ll 1$, 4.26) se torna

$$
\psi_{\omega l} \approx i^{l} A_{\omega l}^{\operatorname{tr}} \frac{(l-1 / 2) !}{\sqrt{\pi}}\left(\frac{2}{M^{2} \omega}\right)^{l}(r-M)^{l},
$$

na qual usamos (4.24) e que

$$
H_{v}^{(1)}(x) \approx-i \frac{(v-1) !}{\pi}\left(\frac{2}{x}\right)^{v}
$$

para $v>0$ e $x \ll 1[46]$.

Outro caminho para se chegar a uma expressão que descreva ondas em baixas frequências próximas ao horizonte a fim de determinamos o coeficiente de transmissão em baixas frequências é resolver a equação (3.2) quando $\omega=0$. No caso extremo a solução é

$$
\psi_{0 l}=b_{1} r(r-M)^{l}+b_{2} r(r-M)^{-l-1} .
$$

Uma vez que ondas com baixas frequências dificilmente ultrapassam o máximo do potencial (3.3) e, portanto, são finitas no horizonte de eventos, concluímos que $b_{2}=0$. A obtenção de 
$b_{1}$ é feita através da comparação da forma de (4.28) quando $r \gg M$ com (4.15). Nesse caso, obtemos que

$$
b_{1}=(-i)^{l+1} A_{\omega l}^{\text {in }} \frac{2^{2 l+1} l !}{(2 l+1) !} \omega^{l+1}
$$

e a solução em baixas frequências então se torna:

$$
\psi_{\omega l} \approx(-i)^{l+1} A_{\omega l}^{\text {in }} \frac{2^{l+1} l !}{(2 l+1) !} \omega^{l+1} r(r-M)^{l} .
$$

Comparando (4.30) no limite em que $r \rightarrow M \operatorname{com}\left(4.27\right.$, obtemos que $T_{\omega l}$, definido por (3.12), é

$$
T_{\omega l} \approx i(-1)^{l+1} \frac{2^{2 l+1} l ! \sqrt{\pi}}{(2 l+1) !(l-1 / 2) !}(M \omega)^{2 l+1} .
$$

Com 3.13, 4.7), 4.31 e lembrando que $\left|R_{\omega l}\right|^{2}+\left|T_{\omega l}\right|^{2}=1$, concluímos que a seção de choque de absorção do campo escalar não massivo em baixas frequências para buracos negros de Reissner-Nordström extremos é

$$
\sigma_{\mathrm{abs}}^{\mathrm{ext}} \approx \pi M^{2} \sum_{l=0}^{\infty}(2 l+1)\left[\frac{2^{2 l+1} l ! \sqrt{\pi}}{(2 l+1) !(l-1 / 2) !}\right]^{2}(M \omega)^{4 l} .
$$

Como no caso de Schwarzschild, aqui o termo não desprezível quando $M \omega \ll 1$ vem da contribuição da onda parcial para $l=0$. Sendo assim temos:

$$
\begin{aligned}
\sigma_{\mathrm{abs}}^{\mathrm{ext}} & \approx \sigma_{\mathrm{abs}}^{\mathrm{ext}(0)} \\
& \approx 4 \pi M^{2}=4 \pi r_{+}^{2}=A_{\mathrm{ext}} .
\end{aligned}
$$

Logo, concluímos que a seção de choque de absorção do campo escalar não massivo em baixas frequências para buracos negros de Reissner-Nordström extremos é igual à área do buraco negro, $A_{\text {ext }}$.

Os resultados destas duas últimas subseções estão de acordo com as pesquisas desenvolvidas em [56] e [57]. Além disso, de acordo com os estudos desenvolvidos nestes artigos, devemos que esperar que a seção de choque de absorção do campo escalar não massivo em baixas frequências no caso de Reissner-Nordström deva ser

$$
\sigma_{\mathrm{abs}}^{\mathrm{RN}} \approx 4 \pi r_{+}^{2}=A_{\mathrm{RN}}
$$

sendo $A_{\mathrm{RN}}$ a área de qualquer buraco negro com $0 \leq q \leq 1$.

\subsubsection{Buracos acústicos canônicos}

A solução da parte radial da equação de Klein-Gordon para o caso de buracos acústicos canônicos, (3.14), quando $\omega=0$ é

$$
\varphi_{0 l}=c_{1} r_{2} F_{1}\left((l+1) / 4,-l / 4,1 / 4, r^{4} / r_{\mathrm{c}}^{4}\right)+c_{2} r_{2}^{4} F_{1}\left((l+4) / 4,(3-l) / 4,7 / 4, r^{4} / r_{\mathrm{c}}^{4}\right),
$$


sendo ${ }_{2} F_{1}$ funções hipergeométricas [46]. Pelo fato de essa solução ser dada em termos de hipergeométricas, a análise de ondas em baixas frequências se revela muito mais complicada que a análise para o caso de buracos negros de Schwarzschild e de Reissner-Nordström extremos. Nesse caso, sabendo que a maior contribuição para a seção de choque de absorção do campo escalar não massivo em baixas frequências vem da onda parcial em que $l=0$, tratamos apenas este termo aqui.

Tomando $\varphi_{00}$, a partir de (4.35) concluímos que

$$
\varphi_{00}=c_{1} r+c_{2} r^{4} \sum_{n=0}^{\infty} \frac{(3 / 4)_{n}(1)_{n}}{(7 / 4)_{n} n !}\left(\frac{r^{4}}{r_{\mathrm{c}}^{4}}\right)^{n}
$$

onde usamos que ${ }_{2} F_{1}(a, 0, c, z)=1$ e $(a)_{n}$ são os símbolos de Pochhammer [46].

Assim como (3.9) resulta em 4.15) quando $\omega r_{*} \ll 1$ para o caso de buracos negros de Reissner-Nordström, de (3.19) obtemos que

$$
\varphi_{\omega 0} \approx-2 i B_{\omega 0}^{\text {in }} \omega r
$$

quando $\omega x \ll 1$. Comparando 4.36 com 4.37 , concluímos que $c_{2}=0$ e $c_{1}=-2 i \omega B_{\omega 0}^{\text {in }}$, de modo que no limite de baixas frequências obtermos 4.37).

No limite em que $r \rightarrow r_{\mathrm{c}} \mathrm{e}|\omega x| \ll 1$, a expressão (3.18) pode ser aproximada por

$$
\varphi_{\omega l} \approx B_{\omega l}^{\mathrm{tr}} .
$$

Tomando 4.37) quando $r \rightarrow r_{\mathrm{c}}$, comparando com 4.38) e lembrando da definição (3.22), concluímos que

$$
D_{\omega 0} \approx-2 i \omega r_{\mathrm{c}}
$$

Usando 3.23, 4.7, 4.39) e lembrando que $\left|C_{\omega l}\right|^{2}+\left|D_{\omega l}\right|^{2}=1$, concluímos que a contribuição da seção de choque de absorção parcial em que $l=0$ para a seção de choque de absorção de buracos acústicos canônicos em baixas frequências é

$$
\begin{aligned}
\sigma_{\mathrm{abs}}^{\mathrm{c}(0)} & =\frac{\pi}{\omega^{2}}\left|D_{\omega 0}\right|^{2} \\
& \approx 4 \pi r_{\mathrm{c}}^{2}=A_{\mathrm{c}} .
\end{aligned}
$$

Portanto, uma vez que a contribuição não desprezível vem da onda parcial em que $l=0$, concluímos que a seção de choque de choque de absorção de buracos acústicos canônicos em baixas frequências é igual à sua área, $A_{\mathrm{c}}$.

\subsubsection{Caso eletromagnético em Schwarzschild}

Para encontrarmos soluções de (3.56) em baixas frequências, vamos escrevê-la como:

$$
\frac{d}{d z}\left[\left(1-z^{2}\right) \frac{d \varphi_{\omega l}^{\lambda}(z)}{d z}\right]+\left[l(l+1)-\frac{2}{z+1}-\omega^{2} M^{2} \frac{(z+1)^{3}}{z-1}\right] \varphi_{\omega l}^{\lambda}(z)=0,
$$


sendo que $z$ é dada por (4.11).

Para modos provenientes do infinito, a solução de (4.41) em baixas frequências, ou seja, fazendo $\omega=0$ nesta equação, é

$$
\varphi_{0 l}^{\lambda}(z) \approx C\left[\mathrm{P}_{l}(z)-\frac{(z-1)}{l(l+1)} \frac{d \mathrm{P}_{l}(z)}{d z}\right]=0,
$$

para $l>0$, com $C$ sendo uma constante de normalização que determinaremos a seguir.

Para encontrarmos $C$, vamos utilizar basicamente o mesmo procedimento das seções anteriores. Nesse caso lembremos que

$$
\mathrm{P}_{l}(z) \approx \frac{(2 l) !}{2^{l}(l !)^{2}} z^{l}
$$

quando $z \gg 1$. Com isto, 4.42 pode ser reescrita como

$$
\varphi_{0 l}^{\lambda}(r) \approx C \frac{l(2 l) !}{2^{l}(l+1)(l !)^{2}}\left(\frac{r}{M}\right)^{l},
$$

para $r \gg r_{\mathrm{S}}$.

Por outro lado, a solução assintótica (3.74) em baixas frequências pode ser escrita da seguinte maneira:

$$
\begin{aligned}
\varphi_{\omega l}^{\lambda} \approx \omega\left\{\left[(-i)^{l+1} B_{\omega l}^{\lambda \text { in }}+i^{l+1} B_{\omega l}^{\lambda \mathrm{ref}}\right] \frac{2^{l} l !}{(2 l+1) !}\left(\omega r_{*}\right)^{l}+\right. \\
\left.i\left[(-i)^{l+1} B_{\omega l}^{\lambda \text { in }}-i^{l+1} B_{\omega l}^{\lambda \mathrm{ref}}\right] \frac{(2 l) !}{2^{l} l !}\left(\omega r_{*}\right)^{-l-1}\right\},
\end{aligned}
$$

onde usamos que as formas das funções de Bessel e Neumann esféricas são dadas por 4.13) e (4.14), quando $\omega r_{*} \ll 1$.

Comparando 4.44) com 4.43, concluímos que $B_{\omega l}^{\lambda \text { ref }} \approx(-1)^{l+1} B_{\omega l}^{\lambda \text { in }}+O(\omega)$ e que

$$
C=(-i)^{l+1} B_{\omega l}^{\lambda \text { in }} \frac{2^{2 l+1}(l+1)(l !)^{3}}{l(2 l) !(2 l+1) !} M^{l} \omega^{l+1} .
$$

Substituindo 4.45 em (4.42) obtemos a seguinte forma para as soluções $\varphi_{\omega l}^{\lambda}$ em baixas frequências:

$$
\varphi_{\omega l}^{\lambda}(z) \approx(-i)^{l+1} B_{\omega l}^{\lambda \text { in }} \frac{2^{2 l+1}(l+1)(l !)^{3}}{l(2 l) !(2 l+1) !} M^{l} \omega^{l+1}\left[\mathrm{P}_{l}(z)-\frac{(z-1)}{l(l+1)} \frac{d \mathrm{P}_{l}(z)}{d z}\right]
$$

Tomando agora (3.73) no limite $\left|\omega r_{*}\right| \ll 1$, obtemos:

$$
\varphi_{\omega l}^{\lambda} \approx \frac{1}{r_{\mathrm{S}}} B_{\omega l}^{\lambda \mathrm{tr}}(1+O(\omega))
$$


A mesma solução nos mesmos limites pode ser obtida quando fazemos $r \approx r_{\mathrm{S}}$ em 4.46 . Sendo assim, obtemos que

$$
\varphi_{\omega l}^{\lambda}=(-i)^{l+1} B_{\omega l}^{\lambda i n} \frac{2^{2 l+1}(l+1)(l !)^{3}}{l(2 l) !(2 l+1) !} M^{l} \omega^{l+1} .
$$

Desde que (4.47) e (4.48) são expressões que descrevem a mesma solução nos mesmos limites, podemos compará-las para obter o coeficiente de transmissão. Fazendo isto, concluímos que em baixas frequências

$$
\left|T_{\omega l}^{\lambda}\right|=\frac{2^{2 l+2}(l+1)(l !)^{3}}{l(2 l) !(2 l+1) !}(M \omega)^{l+1} .
$$

Lembrando que $\left|R_{\omega l}^{\lambda}\right|^{2}+\left|T_{\omega l}^{\lambda}\right|^{2}=1$, das definições 3.76, 3.77) e 3.78, e juntando o com 4.7), concluímos que [16, 54]:

$$
\sigma_{\mathrm{abs}}^{\mathrm{e}-\mathrm{bf}} \approx \frac{4}{3} \pi r_{\mathrm{S}}^{4} \omega^{2}
$$

Para este resultado, consideramos ainda que a maior contribuição para a seção de choque de absorção eletromagnética em baixas frequências vem do termo de onda parcial em que $l=1$. Sendo assim concluímos que a seção de choque de absorção eletromagnética em Schwarzschild vai a zero quando a frequência tende a zero, diferentemente da seção de choque de absorção para o campo escalar não massivo.

\subsection{Espalhamento com pequenos ângulos}

\subsubsection{Schwarzschild}

É possível obtermos resultados analíticos para a seção de choque de espalhamento no limite de pequenos ângulos. Isso pode ser feito basicamente de duas maneiras diferentes. A primeira é expandindo a equação (3.2) em termos de $1 / r$, que é basicamente o limite de campos fracos. Nesse sentido, fazendo $\psi_{\omega l}(r)=f^{-1 / 2} X(r)$, a equação 3.2 pode ser escrita como:

$$
\frac{d^{2} X(r)}{d r^{2}}+\left\{\frac{\omega^{2}}{f^{2}}-\frac{1}{f}\left[\frac{f^{\prime}}{r}+\frac{l(l+1)}{r^{2}}\right]+\frac{\left(f^{\prime}\right)^{2}}{4 f^{2}}-\frac{f^{\prime \prime}}{2 f}\right\} X(r)=0
$$

que também vale para (3.14) desde que substituamos $f$ por $h$ [nesse caso, $\varphi_{\omega l}(r)=h^{-1 / 2} X(r)$ ]. No caso de buracos negros de Schwarzschild $(q=0)$ expandindo (4.51) em termos de $1 / r$ e nos limitando aos casos em que $l \gg M \omega$, obtemos que [15]

$$
\frac{d^{2} X(r)}{d r^{2}}+\left\{\omega^{2}+\frac{4 M \omega^{2}}{r}-\frac{l(l+1)}{r^{2}}\right\} X(r)=0
$$

A equação radial para o espalhamento coulombiano pode ser obtida a partir de 4.52 através das substituições

$$
\frac{2 Z_{1} Z_{2} e^{2} \mu}{\hbar^{2}} \rightarrow 4 M \omega^{2} \text { e } \frac{2 \mu \epsilon}{\hbar^{2}} \rightarrow \omega^{2}
$$


na qual $Z_{1} e$ e $Z_{2} e$ seriam as cargas do alvo e da partícula espalhada e $\mu$ e $\epsilon$ seriam a massa reduzida e a energia do sistema.

A seção de choque de espalhamento coulombiana é [50]

$$
\frac{d \sigma_{\mathrm{el}}^{\mathrm{Cou}}}{d \Omega}=\frac{Z_{1}^{2} Z_{2}^{2} e^{4}}{16 \epsilon^{2} \operatorname{sen}^{4}(\theta / 4)},
$$

que é a seção de choque diferencial do espalhamento de Rutherford. Então, por analogia, a seção de choque de espalhamento do campo escalar não massivo em buracos negros de Schwarzschild no limite de campo fraco e baixas frequências é [15]

$$
\frac{d \sigma_{\mathrm{el}}^{\mathrm{S}-\mathrm{cf}}}{d \Omega}=\frac{M^{2}}{\operatorname{sen}^{4}(\theta / 4)} .
$$

Resultados análogos para partículas não massivas de spins $1 / 2,1$ e 2 podemos ser encontrados da mesma forma. Nesses casos temos [22, 58, 59]:

$$
\frac{1}{M^{2}} \frac{d \sigma_{\mathrm{el}}^{\text {S-cf }}}{d \Omega}=\left\{\begin{array}{lc}
\frac{\cos ^{2}(\theta / 2)}{\operatorname{sen}^{4}(\theta / 2)} & (s=1 / 2) \\
\frac{\cos ^{4}(\theta / 2)}{\operatorname{sen}^{4}(\theta / 2)} & (s=1) \\
\frac{\cos ^{8}(\theta / 2)}{\operatorname{sen}^{4}(\theta / 2)}+\operatorname{sen}^{4}(\theta / 2) & (s=2) .
\end{array}\right.
$$

É interessante notar que fazendo $\theta \rightarrow 0$ nas equações 4.53 ) e (4.54), obtemos:

$$
\left.\frac{d \sigma_{\mathrm{el}}^{\mathrm{S}}}{d \Omega}\right|_{\theta \approx 0} \approx \frac{16 M^{2}}{\theta^{4}} .
$$

Portanto, esta última equação é válida para partículas não massivas de spin 0 [60], 1/2 [22], 1 [42, 54] e 2 [15].

A outra forma de obtermos a expressão 4.55 é tratando perturbativamente geodésicas que passam muito longe do buraco negro. No caso de buracos negros de Schwarzschild essa abordagem pode ser encontrada em [38]. Lá é mostrado que estas geodésicas sofrem um desvio dado por

$$
\Theta(b) \approx \frac{4 M}{b},
$$

que é conhecido como ângulo de deflexão de Einstein. A seção de choque de espalhamento segue diretamente do ângulo de deflexão através da fórmula clássica [40]

$$
\frac{d \sigma_{\mathrm{el}}^{\mathrm{cl}}}{d \Omega}=\frac{b(\theta)}{\operatorname{sen} \theta}\left|\frac{d b}{d \theta}\right| .
$$

Assim, substituindo (4.56) em (4.57), obtemos exatamente (4.55).

Devemos notar que a primeira forma de se chegar a 4.55 , que envolve a aproximação da equação 3.2 no limite de campos fracos, é válida no limite de baixas frequências. A segunda maneira, que envolve o ângulo de deflexão (4.56), é baseada no formalismo de geodésicas e, consequentemente, válida no limite de altas frequências. Assim, concluímos que (4.55) vale para qualquer frequência. 


\subsubsection{Reissner-Nordström}

Não existe interação newtoniana análoga à interação entre buracos negros de Reissner-Nordström e partículas não carregadas (da mesma forma que não existe análogo newtoniano à interação gravitacional causada por buracos negros de Kerr). Considerando apenas a ordem de maior contribuição na métrica de Reissner-Nordström, devemos recair na geometria de Schwarzschild quando $Q^{2} \ll 2 M r$. Note que nesse limite, por 2.2,

$$
f \approx 1-\frac{2 M}{r} \text {. }
$$

Portanto, devemos esperar que as características de espalhamento de buracos negros de ReissnerNordström nesse limite devam ser, em primeira ordem, as mesmas que as obtidas para buracos negros de Schwarzschild. Para buracos negros com intensidades de carga comparáveis a $M$, $Q^{2} \ll 2 M r$ implica em $r \gg M$, que é o limite de campo fraco. Neste caso, concluímos então, que em primeira aproximação, a seção de choque diferencial de espalhamento de buracos negros de Reissner-Nordström no limite de campos fracos deva ser dada por (4.55).

Existem trabalhos, no entanto, onde é calculada a correção que a carga do buraco negro deve introduzir em ordens superiores do ângulo de deflexão para campos fracos e, consequentemente, da seção de choque diferencial de espalhamento para pequenos ângulos. É possível obter esta correção através do estudo de geodésicas. Podemos encontrar resultados como este em alguns artigos, como por exemplo [51], [52] e [53]. Nestes artigos é mostrado que, até a segunda ordem de aproximação, o ângulo de deflexão de geodésicas nulas no espaço-tempo de buracos negros de Reissner-Nordström é dado por:

$$
\Theta(b) \approx \frac{4 M}{b}+\frac{3 \pi}{4}\left(5-q^{2}\right) \frac{M^{2}}{b^{2}} .
$$

O primeiro termo do lado direito desta equação é o ângulo de deflexão de Einstein para buracos negros de Schwarzschild, 4.56.

Nesse caso, usando 4.57) e 4.58), obtemos que a seção de choque diferencial de espalhamento para ângulos pequenos em Reissner-Nordström é

$$
\left.\frac{d \sigma_{\mathrm{el}}^{\mathrm{RN}}}{d \Omega}\right|_{\theta \approx 0} \approx \frac{16 M^{2}}{\theta^{4}}+\frac{15 \pi M^{2}}{4 \theta^{3}}-\frac{3 \pi Q^{2}}{4 \theta^{3}} .
$$

Notamos então que as correções introduzidas devido à carga do buraco negro só aparecem nos termos de segunda ordem tanto do ângulo de deflexão quanto da seção de choque diferencial de espalhamento no limite de campo fraco. Isso mostra que a carga exerce uma influência muito pequena quando estamos muito longe do buraco negro.

\subsubsection{Buraco acústico canônico}

Diferentemente do caso de buracos negros de Schwarzschild no limite de campos fracos, que têm um comportamento similar ao espalhamento coulombiano, buracos acústicos canônicos, 
nesse mesmo limite, não se comportam como nenhum sistema físico conhecido [pelo menos aqueles que possamos encontrar em livros textos - ver seção [5.1, equação [5.5p]. Por isso, aqui optamos por encontrar a seção de choque espalhamento no limite de pequenos ângulos por meio da análise de geodésicas.

A idéia para a abordagem da deflexão de geodésicas que passam muito longe do buraco é tratar a interação entre o buraco e a geodésica como uma perturbação no espaço-tempo plano. Geodésicas no espaço-tempo de Minkowski são encontradas igualando o lado direito da equação (2.22) a zero. Nesse caso, a solução é

$$
u_{0}=\frac{\operatorname{sen} \phi}{b} .
$$

O próximo passo é fazer $u=u_{0}+u_{1}$, sendo que $u_{1}$ é muito menor que $u_{0}$. Nesse caso, mantendo apenas o menor termo no lado direito da equação 2.22, , concluímos que

$$
u \approx \frac{\operatorname{sen} \phi}{b}+\frac{1}{16 b^{5}}\left[\left(9 \cos ^{2} \phi+8-2 \cos ^{4} \phi\right) r_{\mathrm{c}}^{4} \operatorname{sen} \phi-15 r_{\mathrm{c}}^{4} \phi \cos \phi\right] .
$$

Quando o espaço-tempo é plano, $u=1 / r=0$ quando $\phi=0$ ou $\phi=\pi$. No caso de geodésicas no espaço-tempo curvo, $u=0$ quando $\phi=0$ ou $\phi=\pi+\varepsilon$, sendo $\varepsilon$ o ângulo de deflexão. Fazendo então $u=0$ quando $\phi=\pi+\varepsilon$ na equação 4.61 com $\varepsilon \ll 1$, concluímos que o ângulo de deflexão no caso de buracos acústicos canônicos é

$$
\varepsilon=\Theta(b) \approx \frac{15 \pi r_{\mathrm{c}}^{4}}{16 b^{4}} .
$$

Através de 4.57) e 4.62, obtemos que a seção de choque de espalhamento de buracos acústicos canônicos para ângulos pequenos é

$$
\left.\frac{d \sigma_{\mathrm{el}}^{\mathrm{c}}}{d \Omega}\right|_{\theta \approx 0} \approx \frac{\sqrt{15 \pi}}{16} \frac{r_{\mathrm{c}}^{2}}{\theta^{5 / 2}}
$$

Notamos que, assim como no caso de buracos negros de Reissner-Nordström, aqui também a seção de choque diverge para $\theta \rightarrow 0$. Este resultado, no entanto, só é válido no limite de altas frequências. Como veremos no Capítulo 5, onde apresentamos os nossos principais resultados, a seção de choque diferencial de espalhamento de buracos acústicos canônicos é finita no limite $\theta \rightarrow 0$, desde que $\omega$ também seja finito. Este comportamento é completamente diferente do caso de buracos negros de Schwarzschild cuja seção de choque de espalhamento tende ao infinito quando $\theta \rightarrow 0$, independentemente do valor da frequência da onda espalhada $[18,15,61]$.

\subsection{Espalhamento em campo forte - efeito glória}

O efeito glória (no sentido contrário ao de incidência da onda) é decorrente do espalhamento de partículas no sentido oposto ao seu sentido inicial de propagação, mesmo quando o parâmetro 
de impacto não é zero. Esta possibilidade faz surgir padrões de interferência na forma de círculos ou anéis para ângulos de espalhamento próximos a $180^{\circ}$. No caso de buracos negros, este fenômeno está diretamente relacionado à existência de órbitas instáveis para geodésicas nulas.

Em espaços-tempos curvos, e efeito glória é determinado pela fórmula [37, 62]

$$
\left.\frac{d \sigma_{\mathrm{el}}}{d \Omega}\right|_{\theta \approx \pi} \approx 2 \pi \omega b_{\mathrm{g}}^{2}\left|\frac{d b}{d \theta}\right|_{\theta=\pi}\left[J_{2 s}\left(\omega b_{\mathrm{g}} \operatorname{sen} \theta\right)\right]^{2},
$$

na qual $b_{\mathrm{g}}$ é o parâmetro de impacto cuja geodésica sofre deflexão de $180^{\circ}$ e $J_{2 s}(x)$ é a função de Bessel de primeiro tipo e ordem $2 s$, e $s$ é o spin da partícula em questão.

Existem vários parâmetros de impacto para os quais as partículas são espalhadas no sentido oposto ao de incidência. Isso acontece porque, devido a presença da órbita instável, geodésicas podem ter ângulos de deflexão muito grandes. Assim, geodésicas espalhadas no sentido oposto ao de incidência podem ter um ângulo de deflexão dado por $\pi+2 n \pi$, sendo que $n \in \mathbb{N}_{0}$. A definição do efeito glória exige, em princípio, que todas estas contribuições sejam somadas na fórmula (4.64). No entanto, apesar de todos os $b_{\mathrm{g}}$ 's estarem muito próximos uns aos outros, inclusive próximos ao parâmetro de impacto crítico, $b_{\mathrm{c}}$, acontece que

$$
\left|\frac{d b}{d \theta}\right|_{\theta=\pi+2(n+1) \pi} \ll\left|\frac{d b}{d \theta}\right|_{\theta=\pi+2 n \pi} .
$$

Portanto, as contribuições para a intensidade do pico de espalhamento no sentido oposto ao de incidência quando a deflexão é $\pi+2 n \pi, \operatorname{com} n \geq 1$, se tornam desprezíveis quando comparadas ao caso em que a deflexão da geodésica é $\pi$. Pode ser mostrado que, para buracos negros de Schwarzschild, as contribuições subsequentes para o efeito glória são suprimidas por aproximadamente $7 \times 10^{-3}$ com relação as anteriores. A mesma análise nos leva a $10^{-2}$ no caso de buracos negros de Reissner-Nordström extremos e $2 \times 10^{-6}$ para buracos acústicos canônicos.

Os parâmetros $b_{\mathrm{g}}$ e $|d b / d \theta|_{\theta=\pi}$, necessários para determinar completamente a fórmula de aproximação do efeito glória, (4.64), podem ser encontrados a partir de análise das geodésicas. No caso de buracos negros de Reissner-Nordström e buracos acústicos canônicos, estas informações podem ser diretamente obtidas das equações (2.13) e (2.23), respectivamente. A obtenção destes parâmetros pode ser feita desenvolvendo-se estas equações de forma analítica, mas aqui adotaremos os parâmetros obtidos através da suas soluções obtidas numericamente. Para buracos acústicos canônicos isto faz pouca diferença [63], mas no caso de buracos negros de Reissner-Nordström, as aproximações analíticas levam a conclusões errôneas [41].

No caso de buracos negros de Schwarzschild, a forma analítica de parâmetros de impacto próximos à $b_{\mathrm{c}}$ foi primeiramente obtida por C. Darwin através de aproximações de integrais elípticas [64]. A conclusão de Darwin foi que nesse limite,

$$
b \approx\left(5.19+3.48 e^{-\theta}\right) M .
$$

A partir de 4.64) e 4.65), obtemos que

$$
\left.\frac{1}{M^{2}} \frac{d \sigma_{\mathrm{el}}^{\mathrm{S}}}{d \Omega}\right|_{\theta \approx \pi} \approx 26.95 M \omega\left[J_{2 s}(5.34 M \omega \operatorname{sen} \theta)\right]^{2} .
$$


Na Figura 4.1 plotamos o resultado da aproximação do efeito glória para $M \omega=5.0$ e partículas não massivas de spins $0,1 / 2,1$ e 2 obtida por meio do resultado de Darwin. É possível perceber que o único resultado que não vai a zero quando $\theta=180^{\circ}$ é referente ao caso do campo escalar não massivo. No caso do campo eletromagnético podemos compreender este comportamento com base no transporte paralelo do vetor de polarização ao longo da trajetória do raio. Esta mesma idéia pode ser ainda usada para explicação dos resultados para os casos de spin $1 / 2$ e 2 [37].
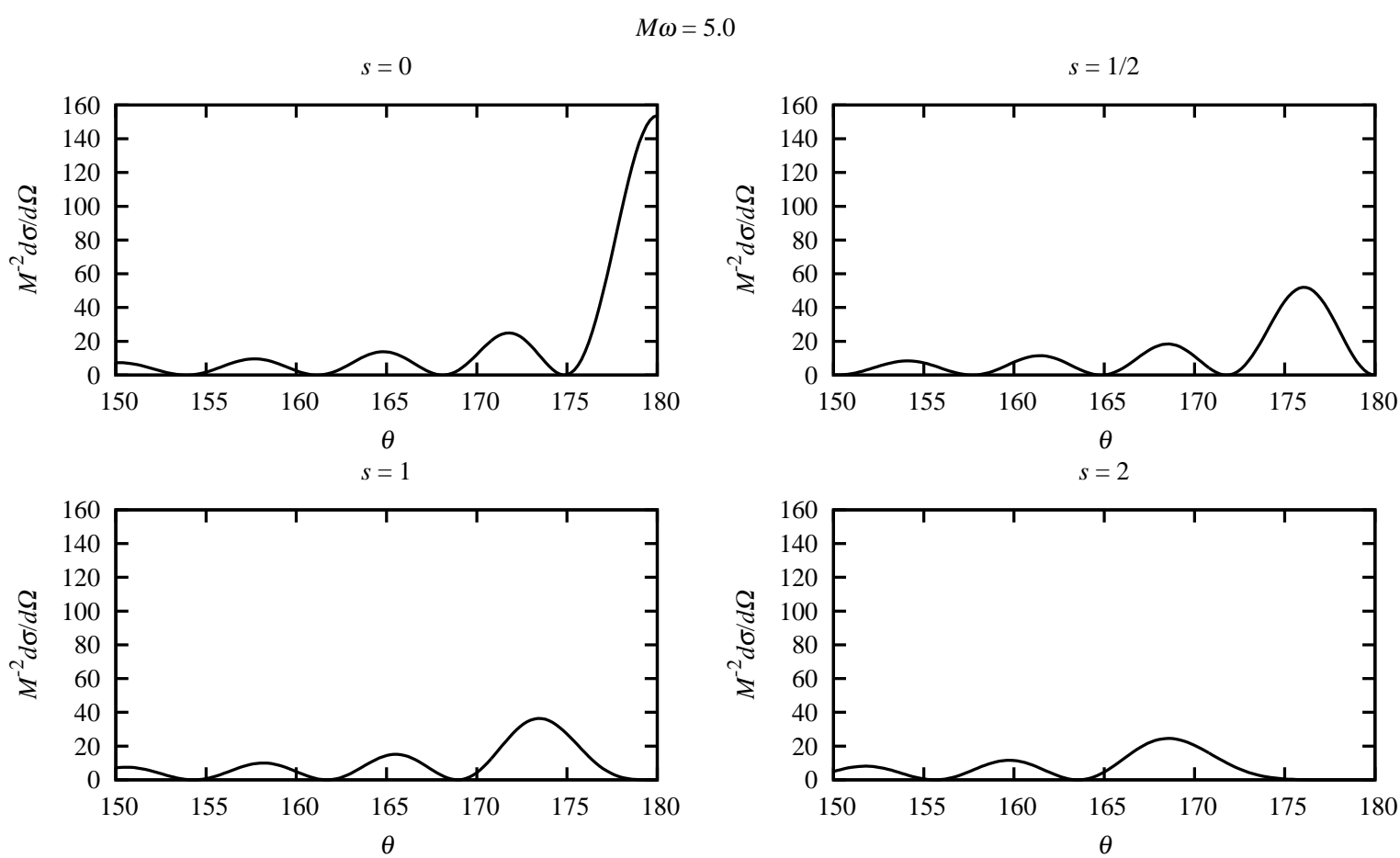

Figura 4.1: Seção de choque diferencial de espalhamento de buracos negros de Schwarzschild para partículas não massivas de spins $0,1 / 2,1$ e 2 obtida com a aproximação do efeito glória. Podemos perceber que todos os resultados são zero para $\theta=180^{\circ}$, exceto para o caso de spin 0 .

Na figura 4.2 mostramos a ilustração de como seria se pudéssemos medir o efeito glória de buracos negros de Schwarzschild em um anteparo.

No caso de buracos negros de Reissner-Nordström, escrevemos a equação 4.64 da seguinte forma

$$
\left.\frac{d \sigma_{\mathrm{el}}^{\mathrm{RN}}}{d \Omega}\right|_{\theta \approx \pi} \approx \mathcal{A}(q)\left[J_{0}\left(\omega b_{\mathrm{g}} \operatorname{sen} \theta\right)\right],
$$

na qual

$$
\mathcal{A}(q)=2 \pi \omega b_{\mathrm{g}}^{2}\left|\frac{d b}{d \theta}\right|_{\theta=\pi}
$$



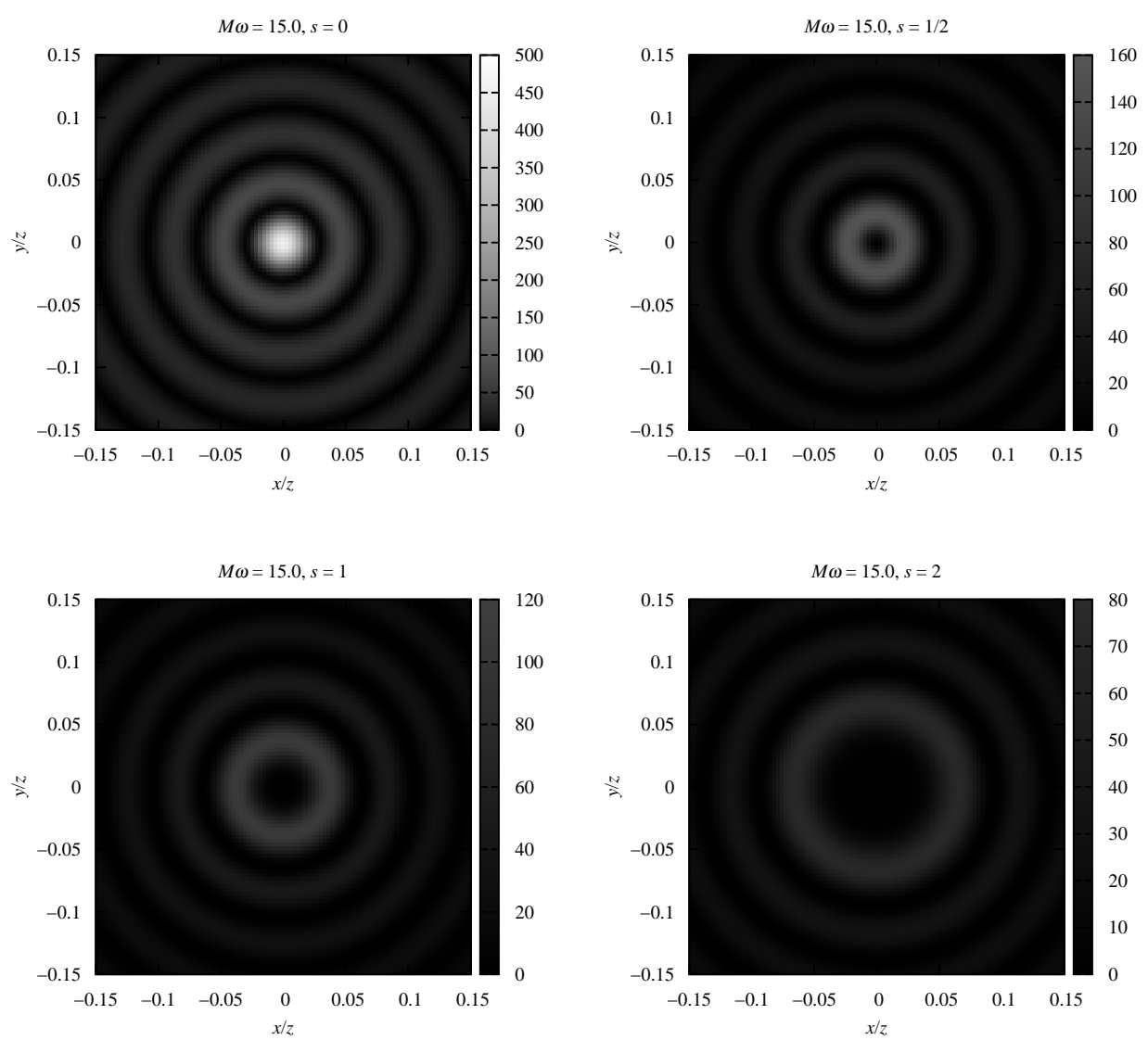

Figura 4.2: Ilustração do efeito glória em buracos negros de Schwarzschild para partículas não massivas de spins $0,1 / 2,1$ e 2 e $M \omega=15.0$. Como dito antes, o único resultado que não vai a zero para $\theta=180^{\circ}$ é referente ao caso de spin 0 . Dessa forma, é observada uma bola branca no centro da figura para este caso, enquanto que nos demais só existem círculos brancos nos padrões de interferência, mas não a bola branca central. 
que nos permite resumir o resultado para diferentes intensidades de carga. Desenvolvendo numericamente a equação (2.13), obtemos o resultado mostrado na Tabela 4.1 .

\begin{tabular}{llr}
\hline \hline$q$ & $b_{\mathrm{g}} / M$ & $\mathcal{A} /\left(M^{3} \omega\right)$ \\
\hline 0 & 5.36 & 30.75 \\
0.8 & 4.76 & 28.70 \\
1 & 4.30 & 28.87 \\
\hline \hline
\end{tabular}

Tabela 4.1: Valores do efeito glória para algumas escolhas da intensidade de carga abordadas durante todo o texto.

No caso de buracos acústicos canônicos, resolvendo numericamente (2.23) obtemos que o efeito glória fica determinado por [63]

$$
\left.\frac{1}{r_{\mathrm{c}}^{2}} \frac{d \sigma_{\mathrm{el}}^{\mathrm{c}}}{d \Omega}\right|_{\theta \approx \pi} \approx 0.0199 \omega r_{\mathrm{c}}\left[J_{0}\left(1.6124 \omega r_{\mathrm{c}} \operatorname{sen} \theta\right)\right]^{2} .
$$

Comparando com o caso de buracos negros de Schwarzschild, 4.66, concluímos que a intensidade do efeito glória de buracos acústicos canônicos é cerca de 170 vezes menor que a de buracos negros de Schwarzschild. Além disso, as larguras dos picos de espalhamento próximos a $180^{\circ}$ de buracos acústicos canônicos são cerca de 1.66 vezes maiores para mesmos valores de $\omega r_{\mathrm{h}}^{1}[65]$.

\footnotetext{
${ }^{1}$ Aqui $r_{\mathrm{h}}$ representa o raio do horizonte de eventos. Assim, $r_{\mathrm{h}}=r_{\mathrm{S}}$ no caso de buracos negros de Schwarzschild e $r_{\mathrm{h}}=r_{\mathrm{c}}$ para buracos acústicos canônicos.
} 


\section{Capítulo 5}

\section{Resultados}

Os nossos principais resultados são apresentados neste capítulo. Como mencionado antes, as soluções das equações radiais dos campos considerados neste trabalho não podem ser escritas em termos de funções especiais conhecidas. Portanto, a determinação de suas propriedades, como comportamento assintótico por exemplo, revela-se difícil, de modo que optamos por analisar o problema de forma numérica. De um modo geral, os métodos analíticos ficam restritos a abordagens com aproximações, o que já apresentamos nos capítulos anteriores. O caso do buraco acústico é uma exceção, visto que parte da solução válida em qualquer limite para a seção de choque diferencial de espalhamento é obtido de maneira analítica.

\subsection{Métodos de cálculo}

Os resultados apresentados neste capítulo são baseados nas soluções numéricas das equações 3.2), 3.14) e 3.56 desenvolvidas de pontos na região $r \gtrsim r_{\mathrm{h}}$ até pontos na região onde $r \gg r_{\mathrm{h}}$. No caso da seção de choque de absorção, uma vez conhecida a solução radial, podemos facilmente relacioná-la aos coeficientes de transmissão e reflexão e assim obtemos os resultados buscados. No caso de buracos negros de Reissner-Nordström para valores de $M \omega$ no intervalo ]0,3], um bom resultado pode ser obtido somando-se as contribuições das ondas parciais de $l=0$ até $l=17$ e desprezando-se os demais. No caso de buracos acústicos canônicos, é suficiente somarmos este mesmo número de contribuições para $\omega r_{\mathrm{c}}$ de 0 a 6 .

A obtenção da seção de choque diferencial de espalhamento é um pouco mais sutil. Vários motivos colaboram para que algumas dificuldades surjam nesse sentido. Em primeiro lugar, uma amplitude de espalhamento precisa envolve a soma de muitas contribuições de onda parcial (cerca de 40 para $\omega r_{\mathrm{h}}=6$ ). Em segundo lugar, as defasagens, que em princípio só podem ser obtidas numericamente, são coeficientes de uma soma na fórmula da seção de choque diferencial de espalhamento [ver (4.3) e 4.4] ] que são multiplicadas, em cada termo da soma, pelos polinômios de Legendre. Neste caso, pequenas imprecisões nos cálculos das defasagens geram grandes erros nas formas das seções de choque diferenciais de espalhamento. Por fim, estas imprecisões se tornam cada vez maiores quanto maiores forem os valores de $l$. 
Devido às imprecisões dos cálculos numéricos, para calcular a seção de choque diferencial de espalhamento de buracos negros de Reissner-Nordström, e também de buracos negros de Schwarzschild para o campo eletromagnético, lançamos mão de um método de convergência [66] baseado nas relações de recorrência dos polinômios de Legendre, dos quais a amplitude de espalhamento depende [ver 4.3] ].

Quanto aos buracos acústicos canônicos, é possível obter resultados muito precisos sem a utilização de métodos de convergência. Apesar de a precisão dos resultados numéricos se tornar menor com o aumento do valor de $l$, podemos contornar este problema utilizando, a partir de determinadas contribuições de onda parcial, valores analíticos da desfasagem. Estes valores analíticos podem ser obtidos basicamente de duas maneiras: (i) usando a descrição semiclássica de espalhamento de Ford e Wheeler [67] e (ii) através da aproximação de Born [45].

Na descrição semiclássica de Ford e Wheeler, cada onda parcial determinada por $l$ e $\omega$ tem um parâmetro de impacto

$$
b=\frac{l+1 / 2}{\omega}
$$

e o ângulo de deflexão é relacionado à defasagem por

$$
\Theta(l)=2 \frac{d}{d l} \operatorname{Re}\left\{\alpha_{l}(\omega)\right\},
$$

sendo que estas equações valem apenas para grandes l's. Usando então a equação (4.62) junto à (5.2), concluímos que para grandes l's, a defasagem de buracos acústicos canônicos vale:

$$
\eta_{l}(\omega) \approx \frac{5 \pi\left(\omega r_{\mathrm{c}}\right)^{4}}{32(l+1 / 2)^{3}},
$$

onde consideramos ainda que, para $l+1 / 2 \gg \omega r_{\mathrm{c}}$, a parte imaginária da defasagem é desprezível.

Para obter a defasagem pela aproximação de Born, expandimos a equação (4.51) em termos de $1 / r$ [porém, com $h$ dado por 2.15 no lugar de $f$ e $\operatorname{com} \varphi_{\omega l}(r)=h^{-1 / 2} X(r)$ ]. Neste caso, obtemos:

$$
\frac{d^{2} X(r)}{d r^{2}}+\left\{\omega^{2}-\frac{l(l+1)}{r^{2}}-U(r)\right\} X(r)=0,
$$

sendo

$$
U(r)=-\frac{2 \omega^{2} r_{\mathrm{c}}^{4}}{r^{4}}+[l(l+1)-6] \frac{r_{\mathrm{c}}^{4}}{r^{6}}
$$

A defasagem na aproximação de Born pode ser dada por [45]:

$$
\delta_{l}^{\mathrm{B}}(\omega) \approx-\omega \int_{0}^{\infty} r^{2}\left[j_{l}(\omega r)\right]^{2} U(r) d r .
$$

Sendo assim, substituindo 5.5 em (5.6) e usando as identidades das funções de Bessel esféricas

$$
\int_{0}^{\infty} x^{-2}\left[j_{l}(x)\right]^{2} d x=\frac{\pi}{(2 l-1)(2 l+1)(2 l+3)}
$$


e

$$
\int_{0}^{\infty} x^{-4}\left[j_{l}(x)\right]^{2} d x=\frac{3 \pi}{(2 l-3)(2 l-1)(2 l+1)(2 l+3)(2 l+5)},
$$

obtemos exatamente 5.3 como maior contribuição para a defasagem.

Conhecendo analiticamente valores aproximados para a defasagem, o que fazemos aqui é calculá-la numericamente até um valor grande de $l$ e depois disso, confrontamos os resultados numéricos com os analíticos. Em um determinado $l$, digamos $l_{\mathrm{m}}$, no qual os valores numéricos e analíticos são basicamente iguais, substituímos o valor da defasagem numérica pela aproximação analítica.

Matematicamente, a amplitude de espalhamento pode ser escrita como:

$$
\begin{aligned}
f_{\omega}(\theta) & =f_{\omega}^{\text {num }}(\theta)+f_{\omega}^{\text {ana }}(\theta) \\
& =\frac{1}{2 i \omega} \sum_{l=0}^{l_{\mathrm{m}}}\left[e^{2 i \eta_{l}(\omega)}-1\right] P_{l}(\cos \theta)+\frac{5 \pi \omega^{3} r_{\mathrm{c}}^{4}}{16} \sum_{l_{\mathrm{m}}+1}^{\infty} \frac{P_{l}(\cos \theta)}{(l+1 / 2)^{2}} .
\end{aligned}
$$

O custo computacional para o desenvolvimento do segundo termo da parte direita de (5.7) é muito pequeno. Portanto, o número máximo de contribuição que consideramos para a seção de choque diferencial de espalhamento pode ser muito grande, fazendo com que nosso resultado se torne muito preciso.

Através de (5.7) é possível provar que a seção de choque de espalhamento de buracos mudos canônicos é finita quando $\theta \rightarrow 0$. Nesse sentido, lembrando que $P_{l}(1)=1$, concluímos que

$$
f_{\omega}^{\mathrm{ana}}(0)=\frac{5 \pi \omega^{3} r_{\mathrm{c}}^{4}}{16} \Psi\left(1, l_{\mathrm{m}}+3 / 2\right),
$$

na qual $\Psi$ é a função poligama [43]. Uma vez que o primeiro termo do lado direito de (5.7) tem um número finito como limite superior do somatório e $f_{\omega}^{\text {ana }}(0)$ é finito, então $f_{\omega}(0)$ também o é. Portanto, a seção de choque de espalhamento para $\theta=0$ é finita. Usando ainda (5.7) é possível obter, computacionalmente, a seção de choque de espalhamento de buracos acústicos canônicos para $\theta=\pi$. Isso acontece porque

$$
\sum_{l=0}^{\infty} \frac{(-1)^{l}}{(l+1 / 2)^{2}}=4 C,
$$

sendo $C$ a constante de Catalan [68]. Uma vez que $P_{l}(-1)=(-1)^{l}$, podemos usar este resultado para obter $f_{\omega}^{\text {ana }}(\pi)$ para qualquer frequência.

\subsection{Seção de choque de absorção}

\subsubsection{Caso escalar em Reissner-Nordström}

A seção de choque escalar de absorção de buracos negros de Reissner-Nordström é obtida relacionando-se a forma assintótica da solução da equação (3.2), que é dada pela equação (3.9), 
com (3.13) e o resultado com 4.7). Boa parte dos resultados desta seção foi desenvolvido primeiramente por Jung e Park em [69].

A Figura 5.1 mostra a seção de choque escalar de absorção parcial para $l=0$ e $q=0,0.8,1$. No limite $M \omega \rightarrow 0$, a única contribuição não desprezível para a seção de choque escalar de absorção vem da onda parcial determinada por $l=0$ [56]. Sabendo disso e lembrando de (4.34), fica claro por que na Figura 5.1 a seção de choque tende à área do horizonte de eventos do buraco negro, $A_{\mathrm{RN}}$, quando $M \omega \rightarrow 0$.

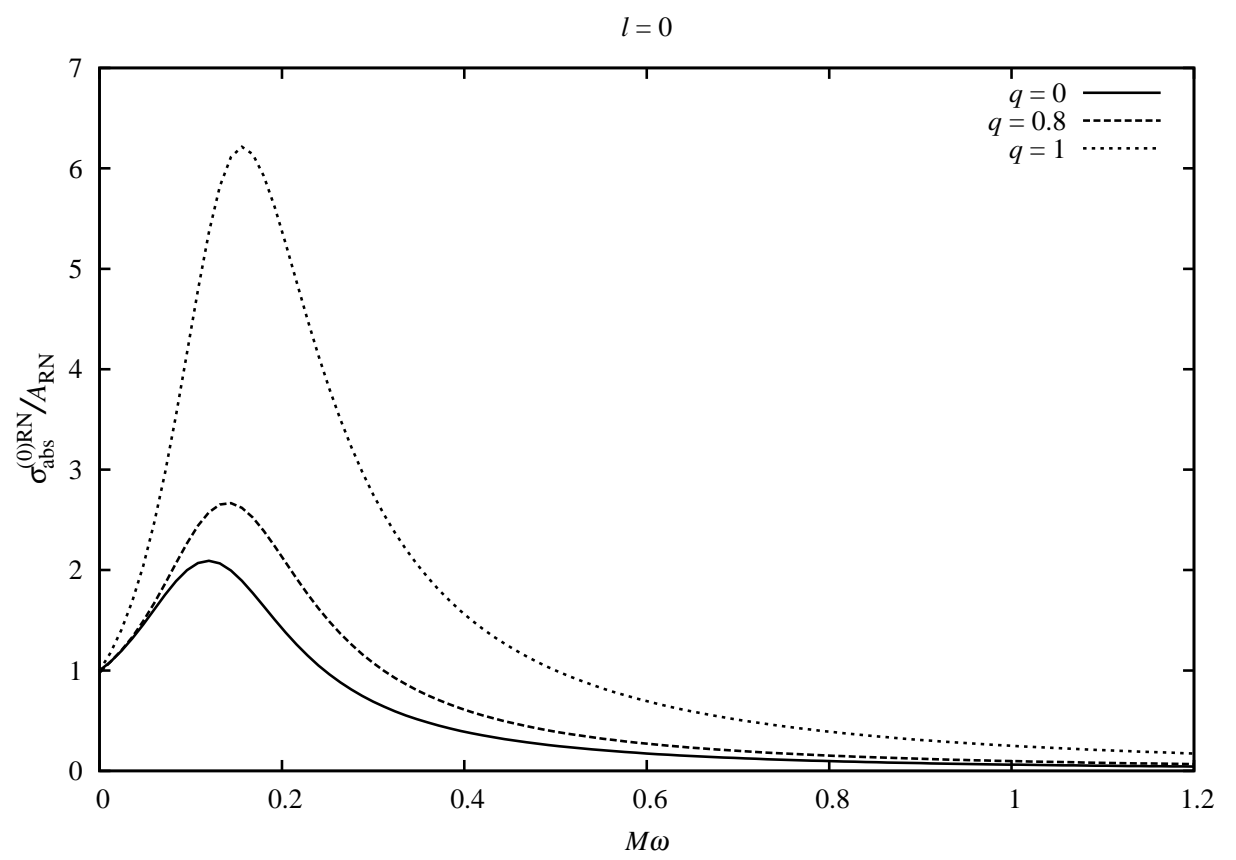

Figura 5.1: Seção de choque escalar de absorção parcial para $l=0$ e $q=0,0.8,1$ comparada com a área do horizonte de eventos do buraco negro, $A_{\mathrm{RN}}$. Vemos que todos resultados tendem a $A_{\mathrm{RN}}$ quando $M \omega \rightarrow 0$. Isso está de acordo com o fato que a contribuição não desprezível para a seção de choque escalar de absorção total em baixas frequências vem da onda parcial em que $l=0$.

Na Figura 5.2 é mostrada a seção de choque escalar de absorção para buracos negros de Reissner-Nordström extremos. As curvas das seções de choque de absorção parciais tem comportamento similar para os outros casos descritos aqui, inclusive o de buracos acústicos canônicos. Como dito antes, podemos observar nesta figura que a contribuição da seção de choque de absorção total no limite de baixas frequências vem quase completamente da seção de choque de absorção parcial na qual $l=0$.

A seção de choque escalar de absorção total para $q=0,0.8,1$ é mostrada na Figura 5.3 Podemos ver nesta figura que a seção de choque de absorção diminui à medida que a intensidade da carga do buraco negro aumenta. Analiticamente, isto foi previsto tanto no limite de altas frequências, 2.10, quanto no limite de baixas frequências, 4.34). É interessante per- 


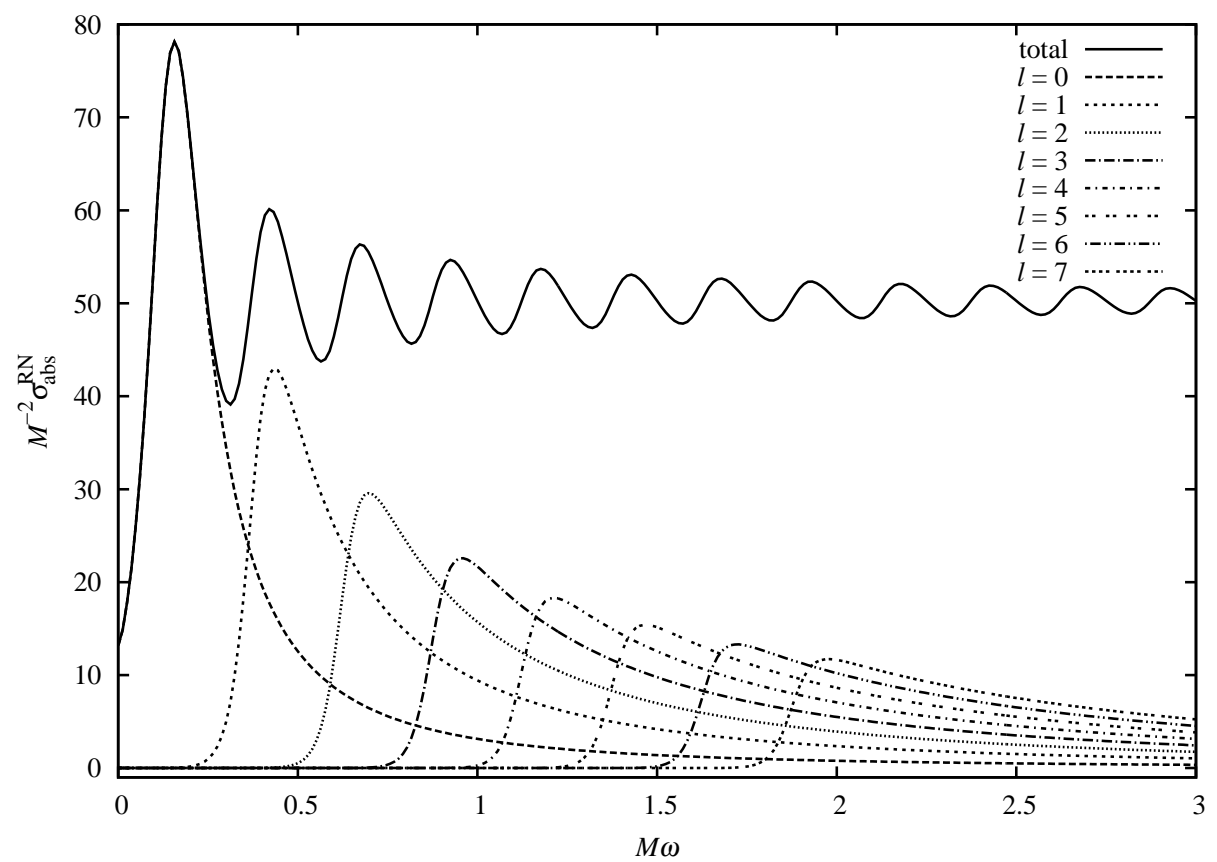

Figura 5.2: Seção de choque escalar de absorção de buracos negros de Reissner-Nordström extremos. Aqui mostramos a seção de choque escalar de absorção total e as suas contribuições parciais até $l=7$. Para a obtenção da curva da seção de choque escalar de absorção total, a contribuição máxima usada foi da onda parcial em que $l=17$. 
ceber que os resultados numéricos estão em excelente acordo com os resultados que foram obtidos analiticamente. Em baixas frequências teremos $\sigma_{\mathrm{abs}}^{\mathrm{RN}-\mathrm{bf}} \approx A_{\mathrm{H}}=\{50.3,32.2,12.6\} M^{2}$, para $q=\{0,0.8,1\}$, respectivamente. Além disso, os resultados numéricos oscilam em torno dos valores das seções de choque de absorção para altas frequências (linhas retas da Figura 5.3. Essa oscilação é devida à soma das seções de choque de absorção parciais. Nesse caso, cada máximo da seção de choque de absorção total corresponde ao máximo de uma seção de choque de absorção parcial.

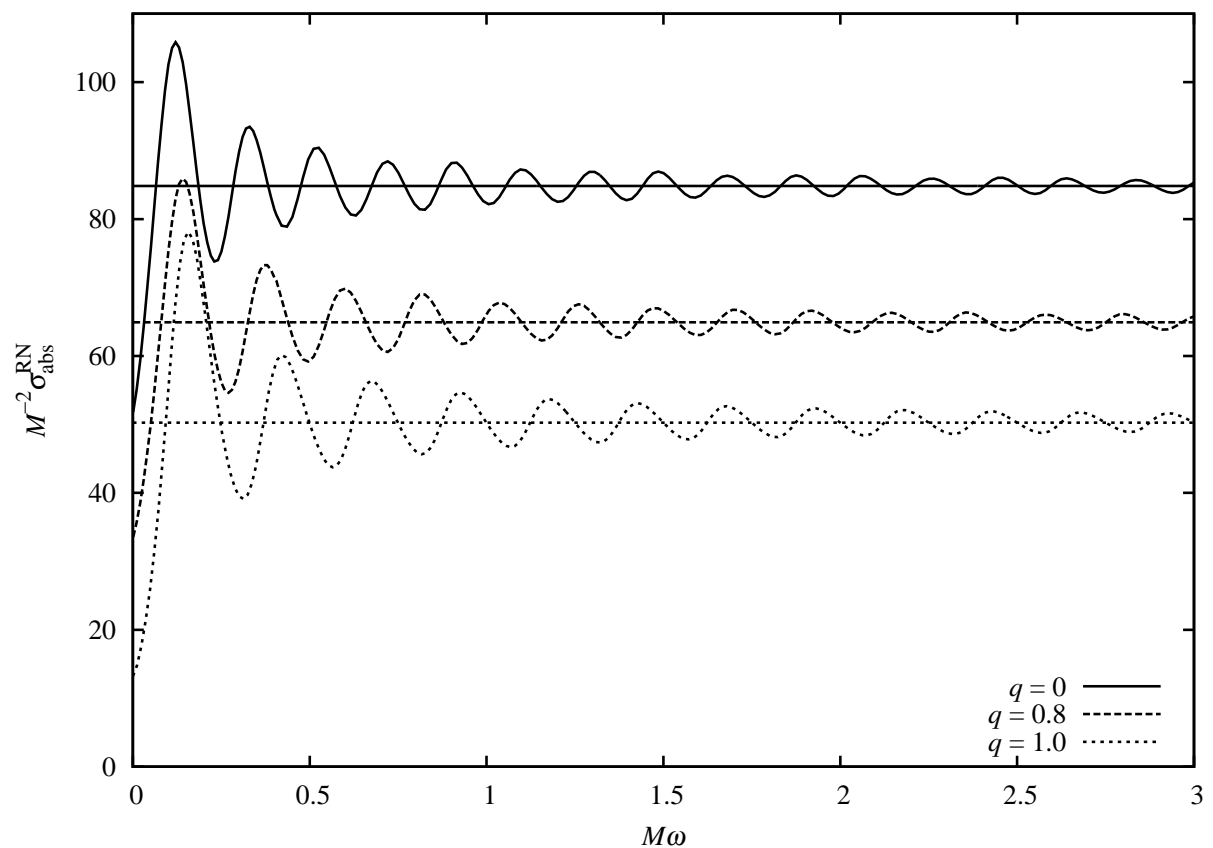

Figura 5.3: Seção de choque escalar total de absorção de buracos negros de Reissner-Nordström para $q=0,0.8,1$. Notamos que à medida que a intensidade da carga do buraco negro aumenta, a seção de choque de absorção diminui. Aqui, as linhas retas são as seções de choque de absorção no limite de altas frequências, dadas pela equação 2.10 .

O resultado mostrado na Figura 5.3 também está de acordo com o comportamento do potencial efetivo (3.3), plotado na Figura 3.2. Lá mostramos que o máximo do potencial efetivo, fixo para cada onda parcial, aumenta quando aumentamos $q$.

\subsubsection{Caso de buracos acústicos canônicos}

A seção de choque de absorção de buracos acústicos canônicos [70] é mostrada na Figura 5.4 . Apesar de na figura mostrarmos a seção de choque de absorção parcial até $l=7$, a soma em 4.7) foi realizada até $l=22$ para obtermos a curva da seção de choque de absorção total. Notamos que para valores de $\omega r_{\mathrm{c}}$ muito próximos de zero, as curvas das seções de choque de absorção total e parcial para $l=0$ coincidem. 


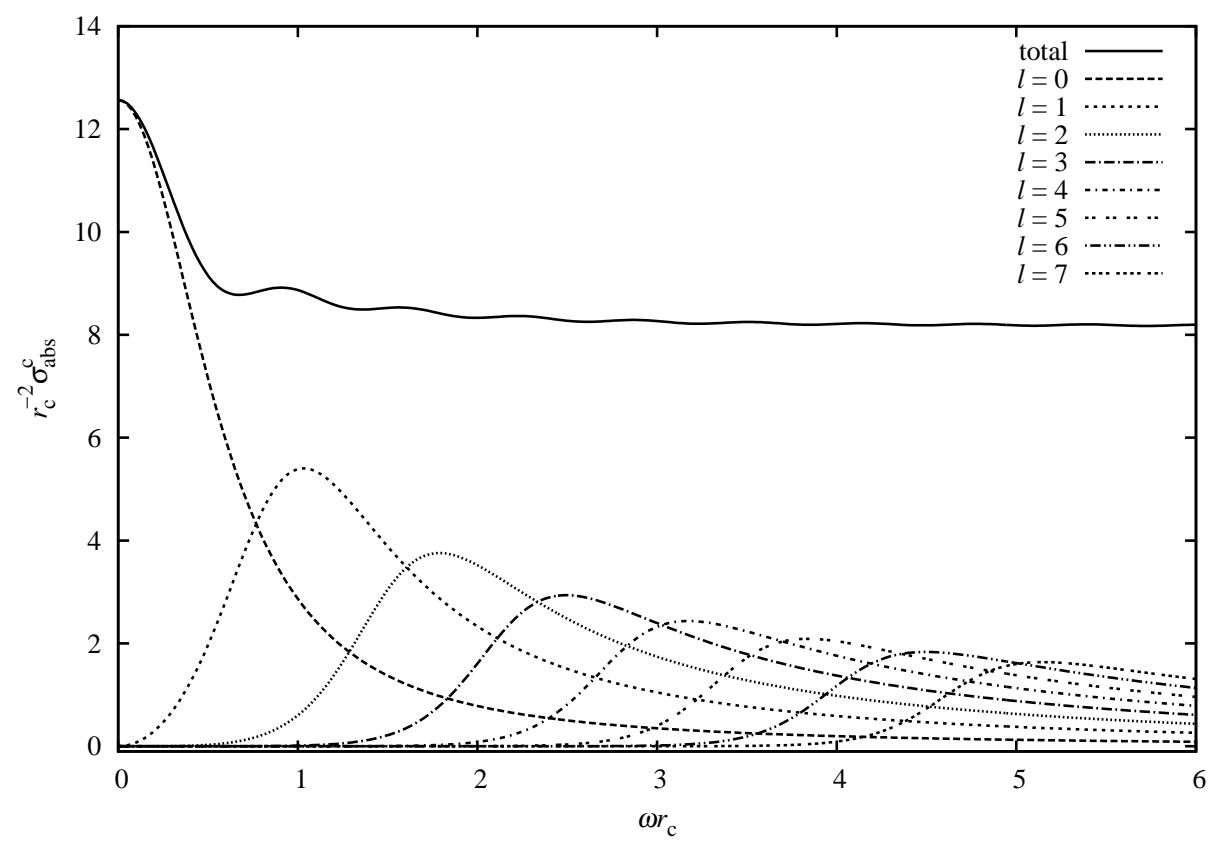

Figura 5.4: Seção de choque de absorção parcial e total de buracos acústicos canônicos. Aqui mostramos as contribuições parciais até $l=7$. Para a obtenção da seção de choque total a soma das seções de choque de absorção parciais foi feita até $l=22$. Note que quando $\omega r_{\mathrm{c}} \rightarrow 0$, as curvas da seção de choque de absorção total e parcial para $l=0$ se sobrepõem. Dessa forma, vemos que no limite de baixas frequências a única contribuição não desprezível para a seção de choque total vem da onda parcial em que $l=0$. 
Na Figura 5.5 mostramos a comparação da seção de choque de absorção de buracos acústicos canônicos com buracos negros de Schwarzschild (caso escalar) [65]. Vemos que quando $\omega r_{\mathrm{h}} \rightarrow 0$, os valores das seções de choque de absorção tendem à área do horizonte de eventos. Isso é esperado, já que de 4.23 e $4.40, \sigma_{\mathrm{abs}} / r_{\mathrm{h}}^{2} \approx 12.6$ para o campo escalar não massivo, independentemente de termos um buraco negro de Schwarzschild ou um buraco acústico canônico. Fora desse limite, a seção de choque de absorção de buracos acústicos canônicos é sempre menor que a de buracos negros de Schwarzschild. Isso é facilmente entendido quando comparamos as métricas destes dois espaços-tempos. Enquanto a métrica de buracos negros de Schwarzschild tende a ser plana com $r^{-1}$, a de buracos acústicos canônicos tende a ser plana $\operatorname{com} r^{-4}$.

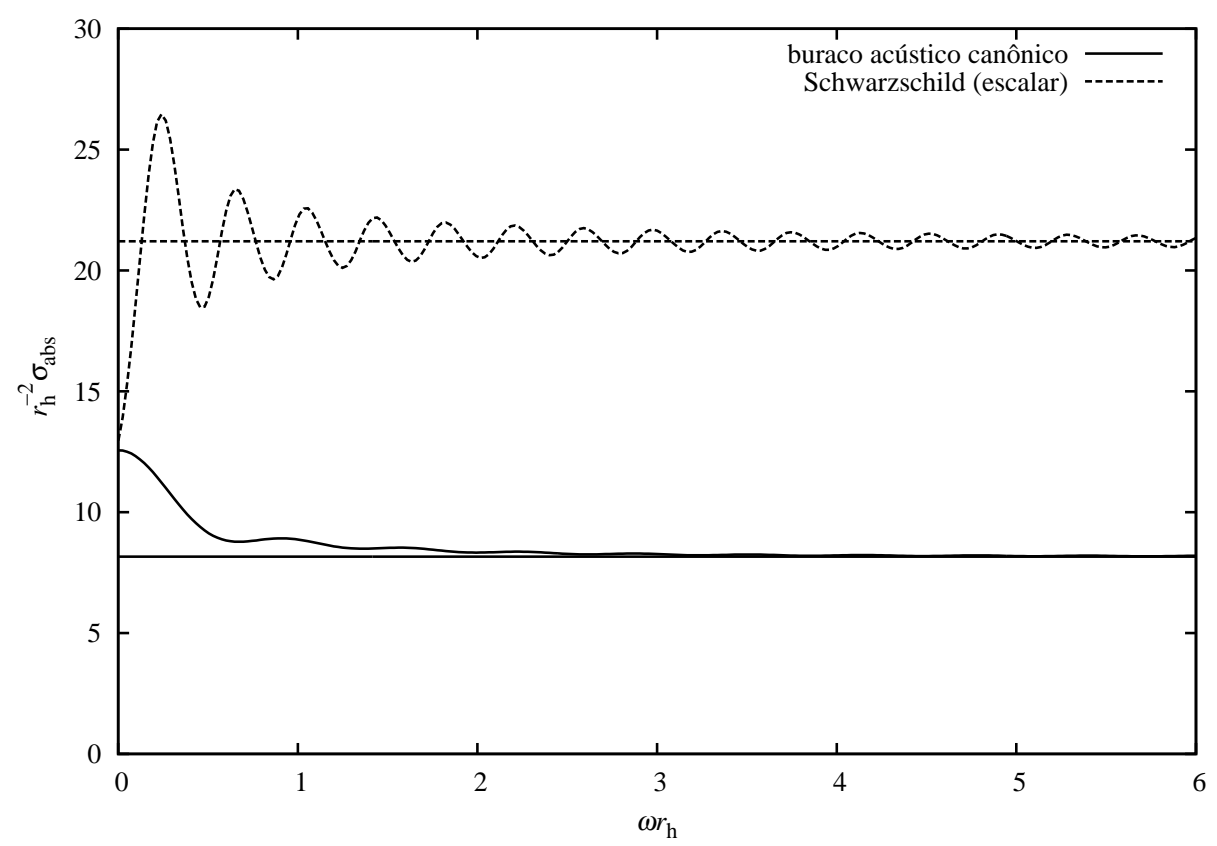

Figura 5.5: Comparação entre a seção de choque de absorção de buracos acústicos canônicos e buracos negros de Schwarzschild (escalar). Notemos que os resultados tendem à área do horizonte de eventos quando $\omega r_{\mathrm{c}} \rightarrow 0$. Fora deste limite, a seção de choque de absorção de buracos acústicos canônicos é sempre menor que a de buracos negros de Schwarzschild. As linhas retas são os limites de altas frequências.

\subsubsection{Caso eletromagnético em Schwarzschild}

As seções de choque de absorção eletromagnética total e parcial de buracos negros de Schwarzschild [21] são mostradas na Figura 5.6. Como no caso escalar, a seção de choque de absorção eletromagnética apresenta oscilações que são devidas às seções de choque de absorção parciais. É interessante notar que para $M \omega<0.2$, aproximadamente, os resultados total e parcial para 
$l=1$ se sobrepõem. Isso foi previsto na subseção 4.2.4. onde vimos que o termo que mais contribui para a seção de choque de absorção eletromagnética em baixas frequências vem da onda parcial com $l=1$.

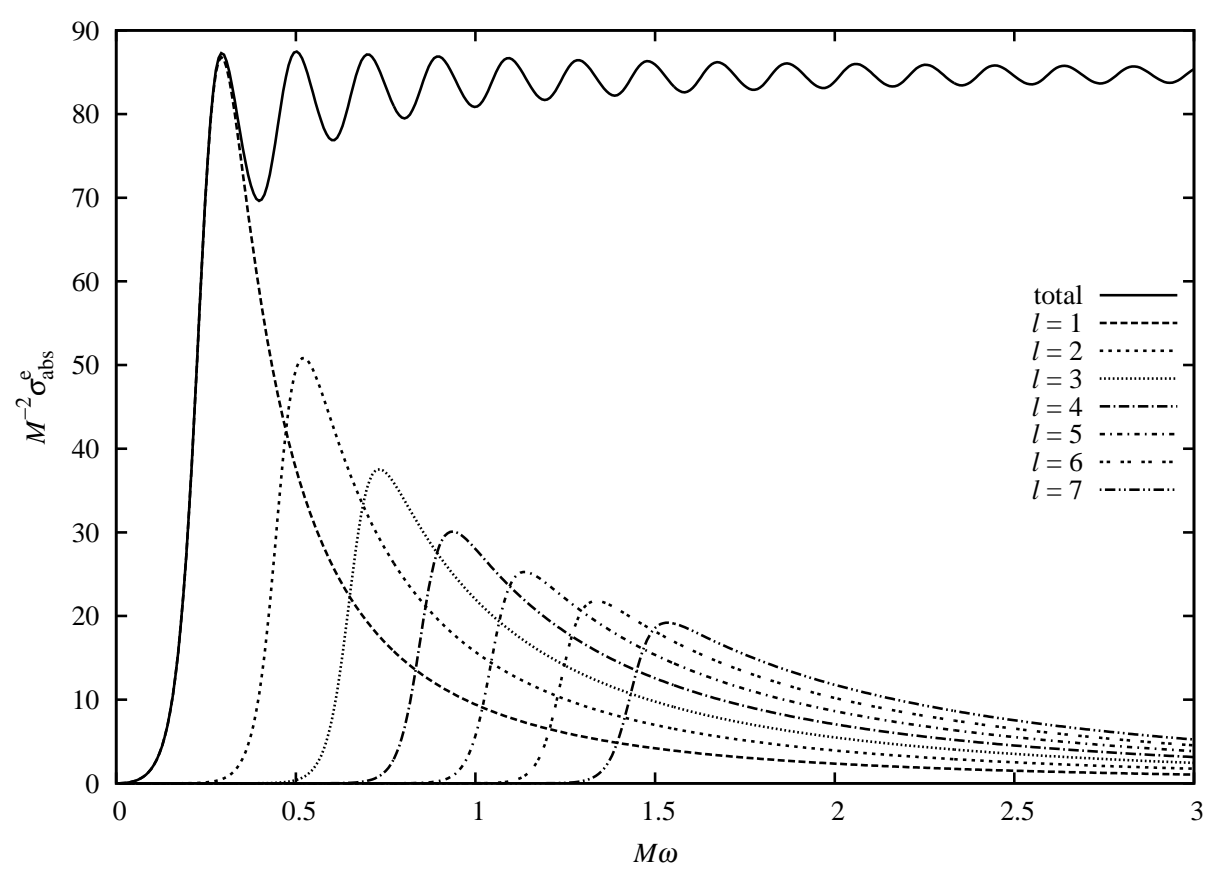

Figura 5.6: Seção de choque de absorção eletromagnética de buracos negros de Schwarzschild. Podemos perceber que para baixas frequências, os resultados total e parcial para $l=1$ se sobrepõem. Além disso, cada máximo da seção de choque de absorção total corresponde ao máximo de uma seção de choque de absorção parcial.

Na Figura 5.7 comparamos as seções de choque de absorção de buracos negros de Schwarzschild para os campos eletromagnético e escalar não massivo. O resultado da ótica geométrica, que no caso de Schwarzschild é obtido fazendo-se $q=0$ em 2.10), e o resultado eletromagnético em baixas frequências, 4.50, também são mostrados. Podemos ver nessa figura que a seção de choque de absorção eletromagnética concorda muito bem com o resultado de baixas frequências e principalmente com a seção de choque de absorção do campo escalar não massivo para valores de $M \omega$ suficientemente grandes. Por ter sido obtido via análise de geodésicas, o valor da seção de choque de absorção no limite da ótica geométrica é válido para qualquer partícula não massiva em altas frequências. Por isso, tanto o resultado escalar quanto o eletromagnético oscilam em torno do resultado da ótica geométrica a medida que a frequência se torna grande. 


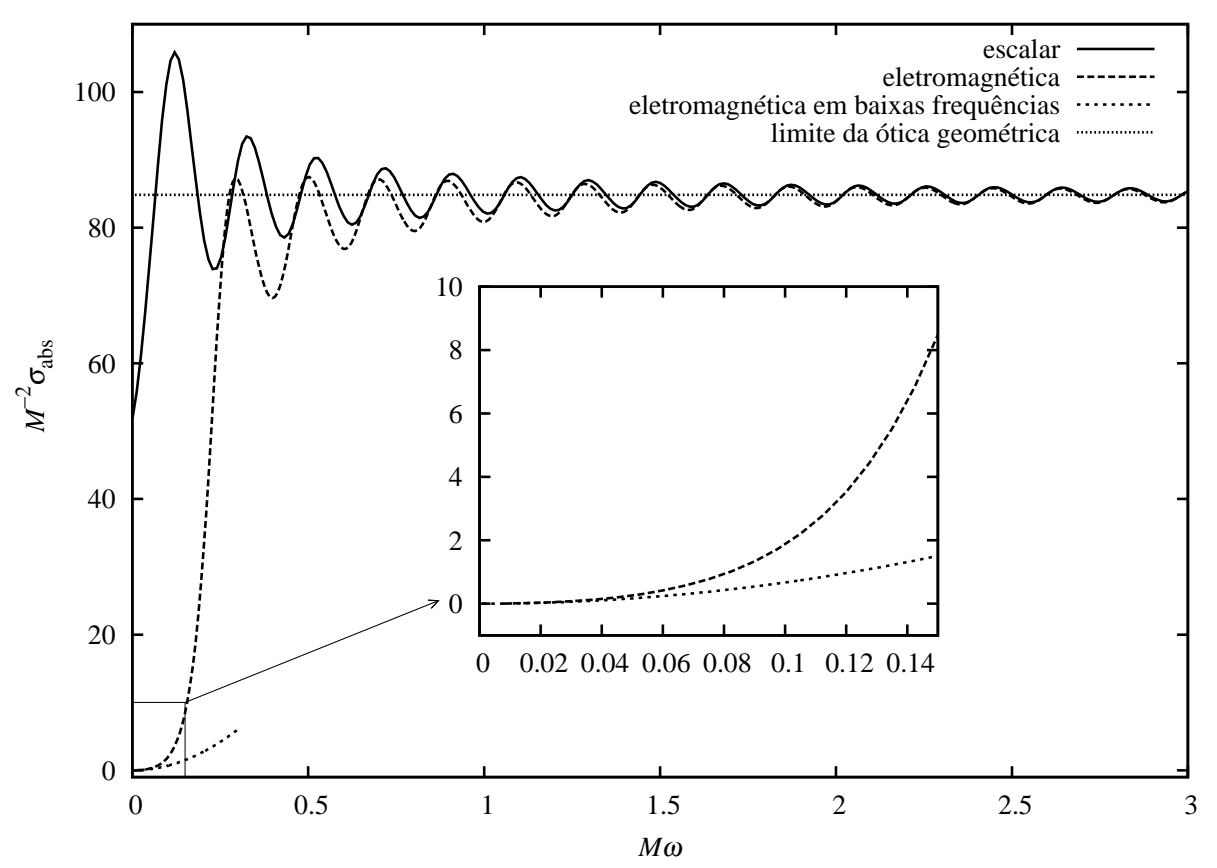

Figura 5.7: Comparação entre a seção de choque de absorção de buracos negros de Schwarzschild para partículas não massivas de spins 0 e 1 . Aqui plotamos também o resultado eletromagnético obtido no regime de baixas frequências e o limite da ótica geométrica. É interessante perceber a concordância do resultado eletromagnético com escalar, que se tornam praticamente iguais para $M \omega>2.5$. Já um pouco antes disso, estes dois resultados oscilam em torno do resultado da ótica geométrica, que é esperado valer no limite de altas frequências para partículas não massivas, independentemente do spin. 


\subsection{Seção de choque de espalhamento}

Os resultados apresentados nesta seção foram todos publicados em [41], que trata do espalhamento escalar por buracos negros de Reissner-Nordström, em [63], que trata do espalhamento por buracos acústicos canônicos e em [23] onde realizamos o cálculo da seção de choque diferencial eletromagnética de buracos negros de Schwarzschild.

\subsubsection{Caso escalar em Reissner-Nordström}

A Figura 5.8 mostra as seções de choque escalares diferenciais de espalhamento (em escala logarítmica) de buracos negros de Reissner-Nordström para $q=0,0.8,1$ e $M \omega=3.0$. Na mesma figura plotamos os resultados da aproximação para o efeito glória, dado pela equação (4.67) (complementado pela Tabela 4.1) no caso de buracos negros de Reissner-Nordström. Notamos que, apesar da aproximação para o efeito glória ser esperada valer apenas para $M \omega \gg$ 1 , nesta figura podemos perceber que os valores numéricos estão muito próximos dos resultados do efeito glória para $\theta>160^{\circ}$.

As seções de choque diferenciais de espalhamento escalar de buracos negros de ReissnerNordström com $q=0,0.8,1$ são comparadas para $M \omega=1.0$ e 3.0 na Figura 5.9. Nesta figura podemos perceber que não há uma variação monotônica da intensidade de espalhamento quando aumentamos a intensidade da carga do buraco negro como acontece com a seção de choque de absorção que é mostrada na Figura 5.3. Por outro lado, as larguras das franjas de interferência evidentemente aumentam quando aumentamos a intensidade da carga do buraco negro. Este comportamento pode ser previsto com base na fórmula do efeito glória (4.67). De acordo com aquela equação, as distâncias entre os picos da seção de choque diferencial de espalhamento variam inversamente a $b_{\mathrm{g}}$. Como podemos ver na Tabela 4.1, $b_{\mathrm{g}}$ diminui à medida que $q$ cresce.

É interessante analisarmos a magnitude da seção de choque diferencial de espalhamento escalar de buracos negros de Reissner-Nordström para o ângulo de $180^{\circ}$ quando variamos a intensidade da carga do buraco negro. Esta análise é mostrada na Figura 5.10. Nessa figura plotamos a intensidade do efeito glória obtida através da substituição de valores numéricos da equação (2.13) na equação (4.64), de valores obtidos analiticamente das mesmas equações, e através do resultado numérico para a seção de choque de espalhamento quando $M \omega=1.0,3.0 \mathrm{e}$ 5.0. Da Figura 5.10 podemos ver que o resultado analítico pode nos levar a conclusões errôneas. Segundo este resultado, a intensidade do efeito glória deveria sempre diminuir à medida que a intensidade de carga do buraco negro aumentasse. Entretanto, através da análise numérica do efeito glória vemos que a intensidade do efeito não tem um decréscimo monotônico. Quanto aos resultados numéricos baseados no método de ondas parciais, eles oscilam em torno do resultado numérico do efeito glória. Portanto, a concordância destes resultados numéricos é muito boa mesmo em frequências que não são altas.

Algo que poderíamos nos perguntar ainda é por que apesar de $b_{\mathrm{g}}$ diminuir com a carga, a intensidade do efeito glória não diminui monotonicamente com essa diminuição? De (4.64) concluímos que para entender como a intensidade do efeito glória deve variar, além de analisar 

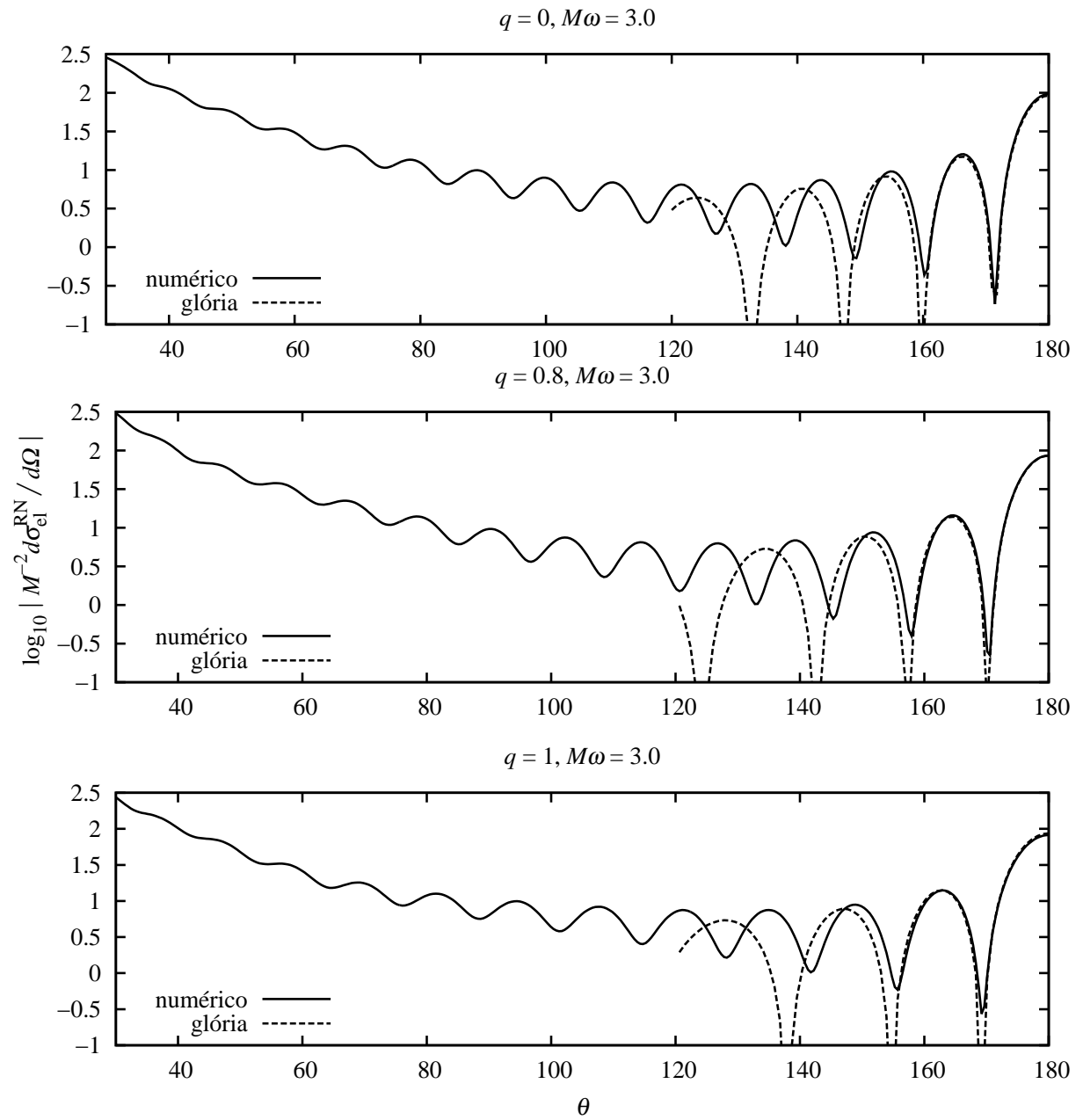

Figura 5.8: Seção de choque escalar diferencial de espalhamento de buracos negros de Reissner-Nordström para $M \omega=3.0$ e $q=0,0.8,1$. As curvas tracejadas representam a aproximação obtida do efeito glória. Notamos que existe uma excelente concordância dos resultados numéricos com esta aproximação quando $\theta \approx 180^{\circ}$. Isto ocorre mesmo para um valor de $M \omega$ relativamente baixo (considerando-se o limite $M \omega \gg 1$, no qual a aproximação do efeito glória deve ser válida) como é o caso do escolhido para estes gráficos. 


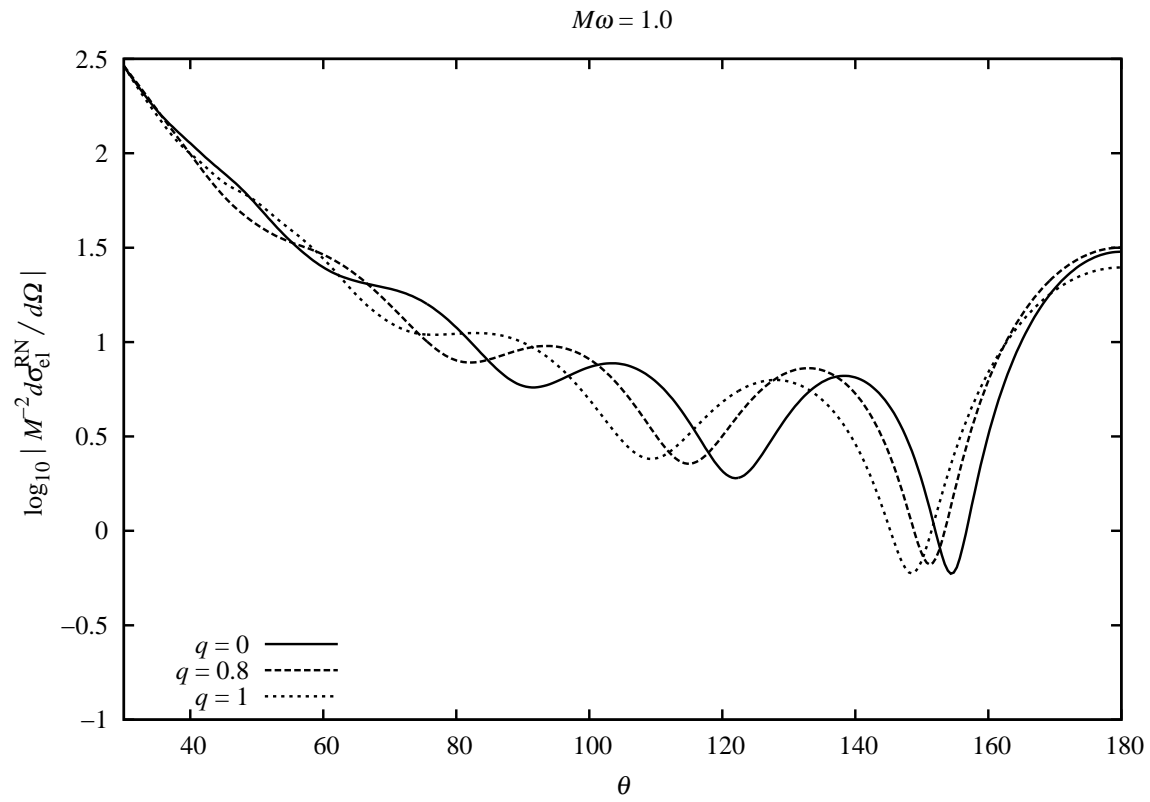

(a) Seção de choque diferencial de espalhamento do campo escalar não massivo em Reissner-Nordström com $q=0,0.8,1$ para $M \omega=1.0$.

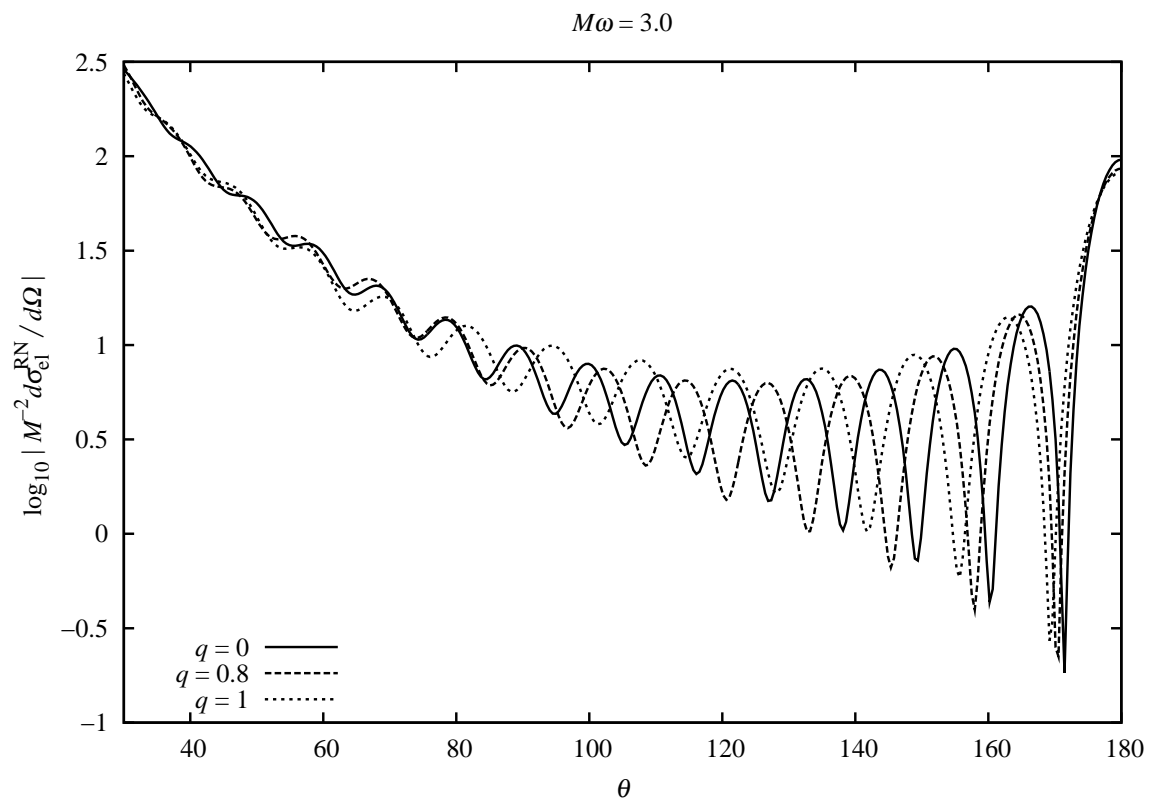

(b) Seção de choque diferencial de espalhamento do campo escalar não massivo em Reissner-Nordström com $q=0,0.8,1$ para $M \omega=3.0$.

Figura 5.9: Nesta figura, não é possível determinar um padrão na variação da intensidade dos resultados para diferentes valores de $q$. Já as larguras das franjas de interferência claramente ficam maiores quando $q$ cresce. 


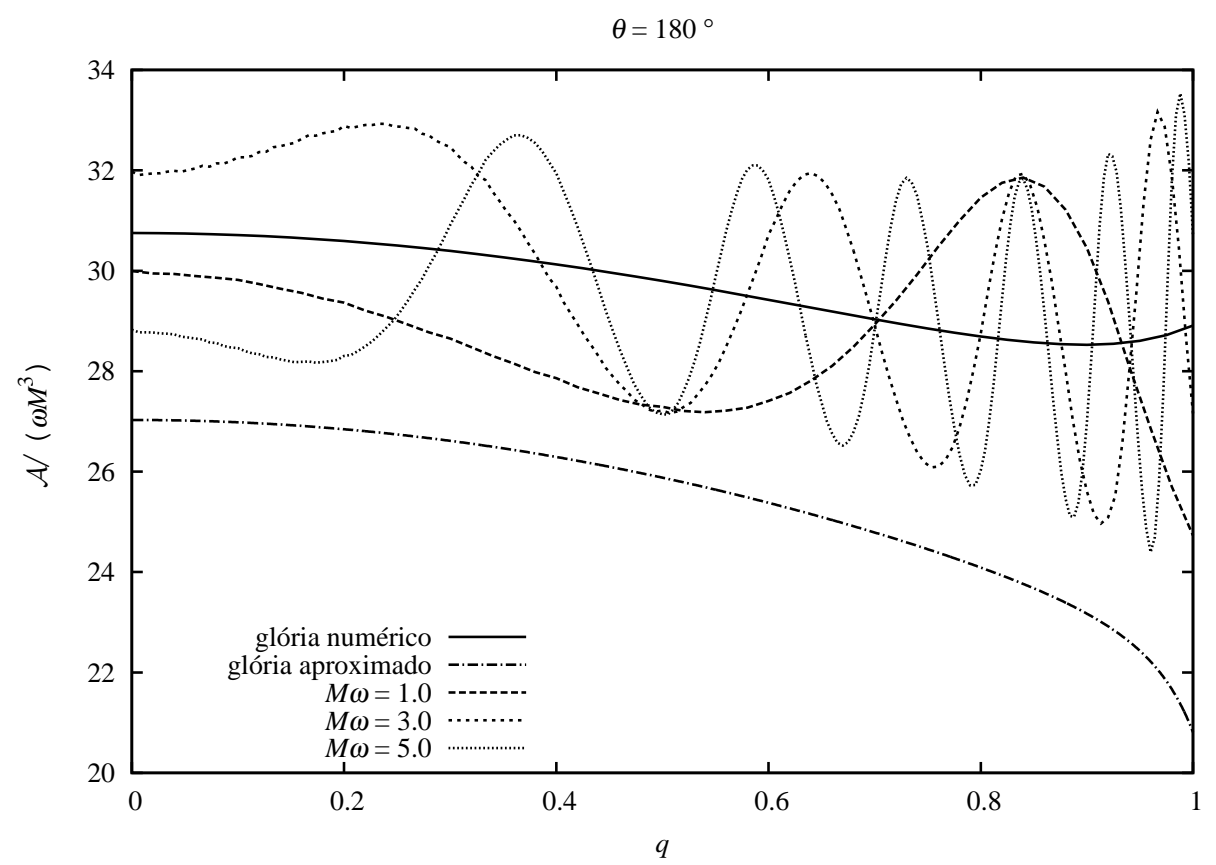

Figura 5.10: Intensidade do espalhamento escalar de buracos negros de Reissner-Nordström no ângulo $180^{\circ}$. Vemos que enquanto a aproximação analítica dos parâmetros de efeito glória resulta em um decréscimo na intensidade de espalhamento, o resultado numérico para este efeito e os resultados numéricos baseados no método de ondas parciais indicam que a intensidade não diminui monotonicamente com o crescimento de $q$. Além disso, os resultados numéricos baseados no método de ondas parciais oscilam em torno do resultado numérico do efeito glória, mostrando uma boa concordância dos nossos resultados numéricos com a fórmula (4.67). 
$b_{\mathrm{g}}$ temos que analisar $|d b / d \theta|_{\theta=\pi}$. Na Figura 5.11 mostramos $b_{\mathrm{g}}$ e $|d b / d \theta|_{\theta=\pi}$ plotados como funções da intensidade de carga do buraco negro, $q$. Como podemos ver nesta figura, apesar de $b_{\mathrm{g}}$ decrescer com o aumento de $q,|d b / d \theta|_{\theta=\pi}$ aumenta. Este crescimento de $|d b / d \theta|_{\theta=\pi}$ impede que a intensidade do efeito glória diminua monotonicamente com o aumento de $q$. Por outro lado, a comparação destes parâmetros calculados numericamente e analiticamente nos revelam que o erro notado no cálculo analítico da intensidade do efeito glória (Figura 5.10) se concentra mais no cálculo de $|d b / d \theta|_{\theta=\pi}$. Além disso, podemos notar ainda que o erro aumenta com o aumento da intensidade de carga, $q$. Em números, os erros para o caso de buracos negros de Schwarzschild são aproximadamente de $0.2 \%$ e $12.3 \%$ para $b_{\mathrm{g}}$ e $|d b / d \theta|_{\theta=\pi}$, respectivamente. No caso de buracos negros de Reissner-Nordström extremos, estes erros são, respectivamente, em torno de $1.0 \%$ e $39.1 \%$.

\subsubsection{Caso de buracos acústicos canônicos}

A seção de choque diferencial de espalhamento de buracos acústicos canônicos é plotada na Figura 5.12. Nesta figura vemos que à medida que a frequência aumenta, o comprimento das franjas de interferência diminui. Isso pode ser previsto com base na fórmula do efeito glória, (4.68). Algo interessante nesta figura é que a seção de choque de espalhamento para $\theta=0$ é finita. Isso está de acordo com as equações (5.7) e (5.8), que juntas provam que a seção de choque de espalhamento de buracos acústicos canônicos em $\theta=0$ é finita desde que $\omega r_{\mathrm{c}}$ seja finito.

A Figura 5.13 mostra a seção de choque diferencial de espalhamento de buracos acústicos canônicos para os ângulos $\theta=0$ e $\theta=180^{\circ}$. Podemos ver que no primeiro caso, a curva cresce muito rapidamente com o crescimento de $\omega r_{\mathrm{c}}$. Isso é esperado uma vez que, no limite de altas frequências, obtivemos que a seção de choque de espalhamento diverge quando $\theta \rightarrow 0$, de acordo com a equação (4.63). Quanto à seção de choque diferencial de espalhamento para $\theta=180^{\circ}$, podemos ver que seu comportamento tende a ser o mesmo da seção de choque de espalhamento obtida para o efeito glória (4.68), desde que frequência não seja pequena. A fórmula do efeito glória, (4.64), é semiclássica, de modo que devemos esperar que ela se aproxime cada vez mais do resultado correto quanto maior for o valor da frequência.

Uma vez que a seção de choque diferencial de espalhamento de buracos acústicos canônicos não é infinita para nenhum valor de $\theta$, a seção de choque de espalhamento total, obtida por meio da integração de (4.2) no ângulo sólido $d \Omega$, que resulta na equação (4.5), é finita. Este resultado é completamente diferente do obtido no caso de buracos negros de Schwarzschild (e de Reissner-Nordström) que têm a seção de choque diferencial de espalhamento infinita para $\theta \rightarrow 0$ [18, 61]. Na Figura 5.14 plotamos a seção de choque de espalhamento e a seção de choque total de buracos acústicos canônicos. O comportamento da seção de choque de espalhamento é diferente da seção de choque de absorção, plotada na Figura 5.4. Enquanto a seção de choque de absorção parcial diminui, a seção de choque de espalhamento parcial aumenta com o aumento de $l$. Além disso, a seção de choque de espalhamento total cresce aproximadamente de forma linear com $\omega r_{\mathrm{c}}$, enquanto que a seção de choque de absorção tende à um valor fixo, (2.20), que é o resultado de altas frequências. A seção de choque total tem o 


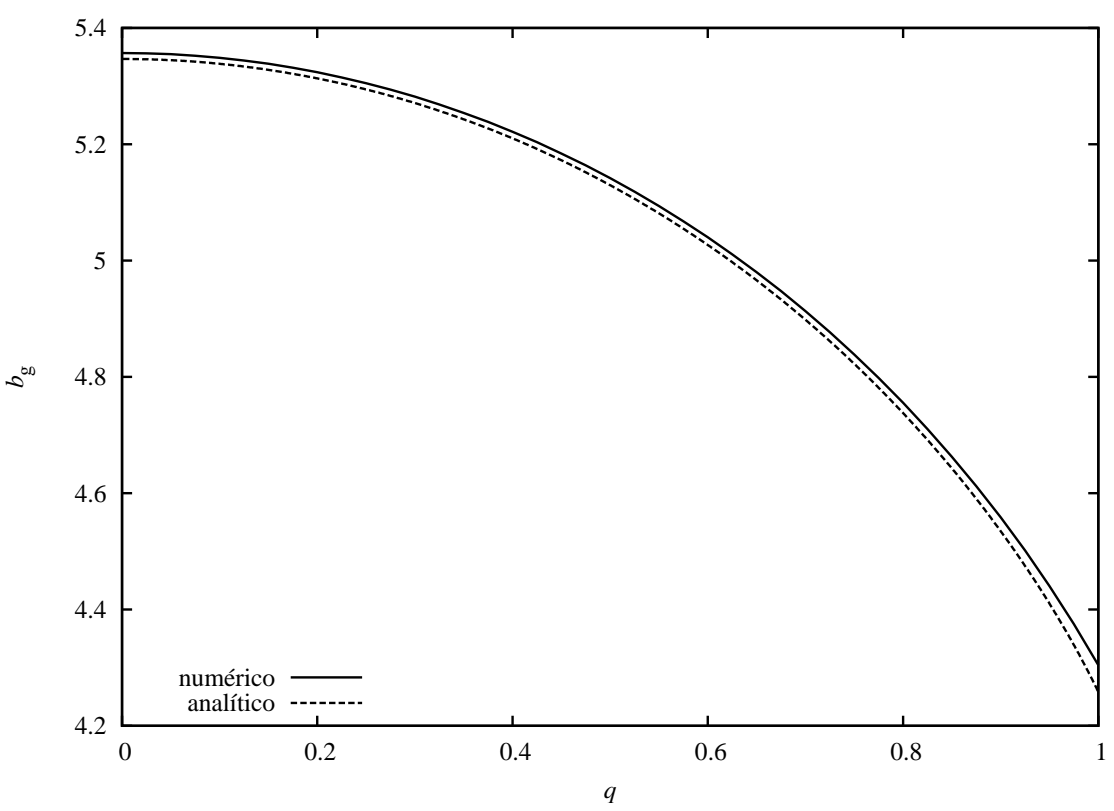

(a) Parâmetro de impacto para o ângulo de deflexão de $180^{\circ}$.

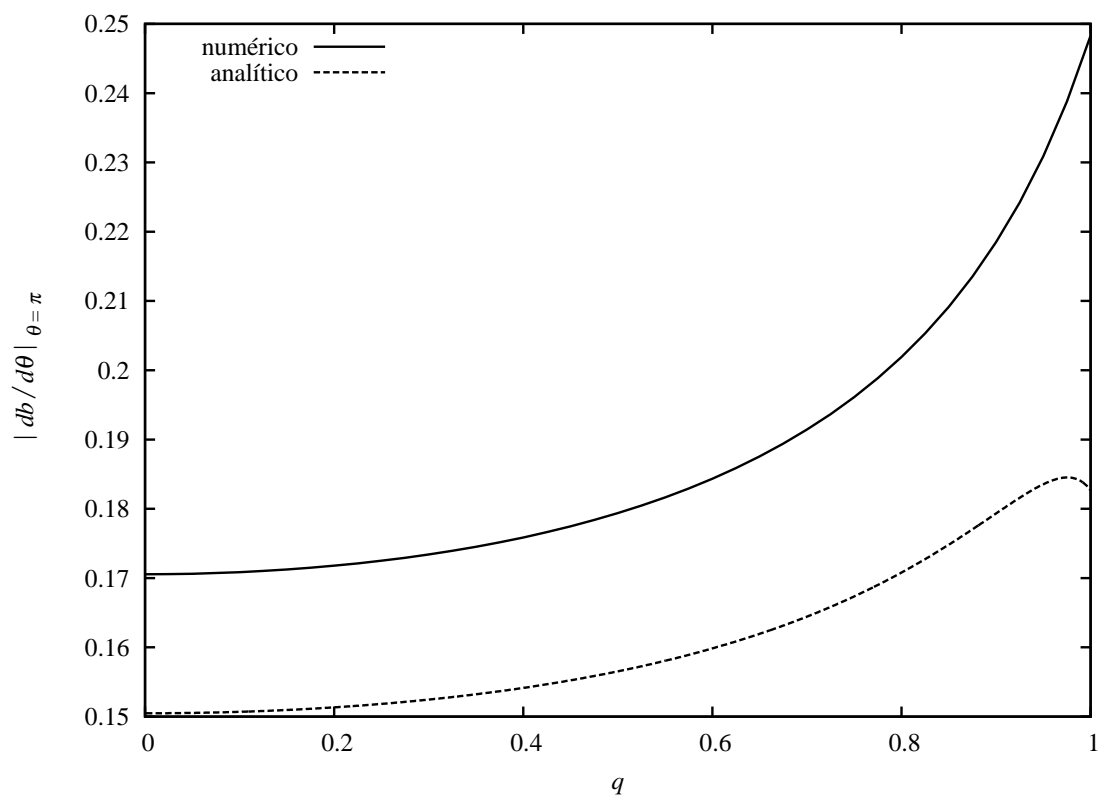

(b) Derivada do parâmetro de impacto com relação ao ângulo de deflexão para $\theta=$ $180^{\circ}$.

Figura 5.11: Comparação dos parâmetros para o efeito glória em buracos negros de ReissnerNordström obtidos analitica e numericamente. As linhas contínuas representam os cálculos numéricos e as tracejadas mostram os resultados analíticos. Podemos ver que $|d b / d \theta|_{\theta=\pi}$, plotado no gráfico (b), apresenta a maior diferença entre os resultados e que esta diferença cresce $\operatorname{com} q$. 


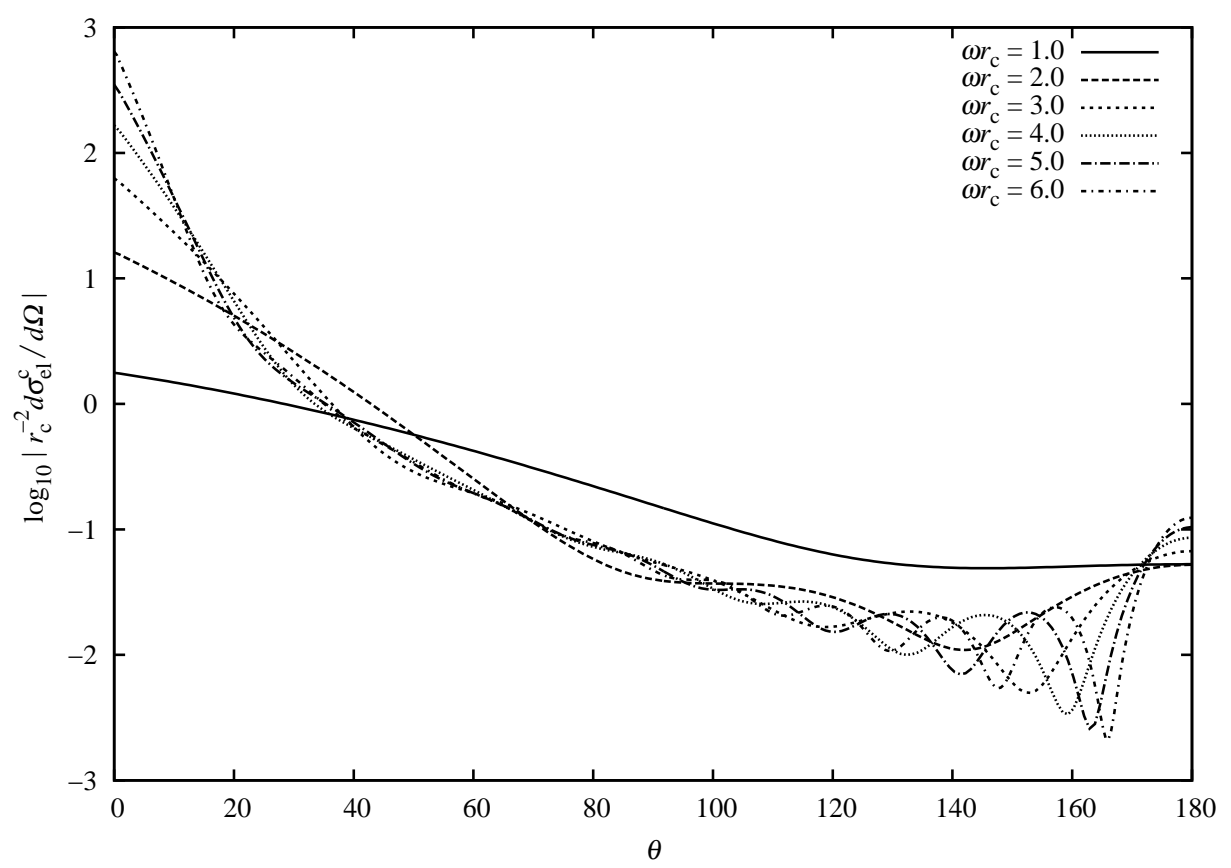

Figura 5.12: Seção de choque diferencial de espalhamento de buracos acústicos canônicos para valores de $\omega r_{\mathrm{c}}$ de $1 \mathrm{a} 6$. Podemos perceber que a seção de choque diferencial de espalhamento é finita em todo o intervalo de $[0,180]$ do ângulo de espalhamento, $\theta$. Notamos também que as larguras dos padrões de interferência diminuem quando aumentamos $\omega r_{\mathrm{c}}$. 


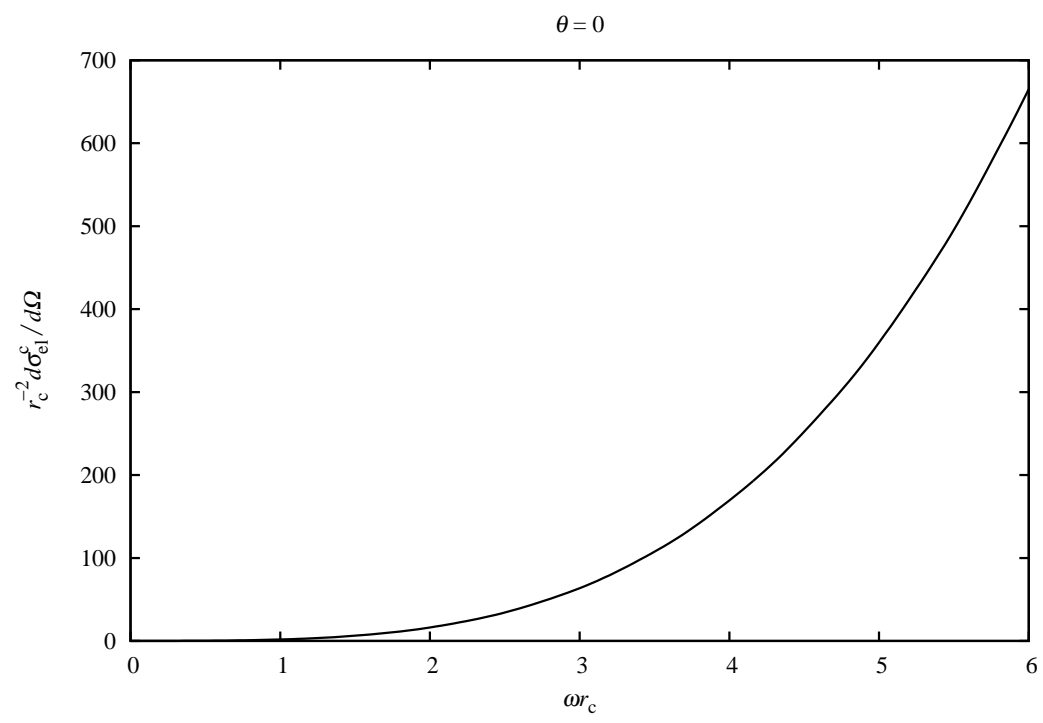

(a) Seção de choque diferencial de espalhamento de buracos acústicos canônicos no eixo para $\theta=0$.

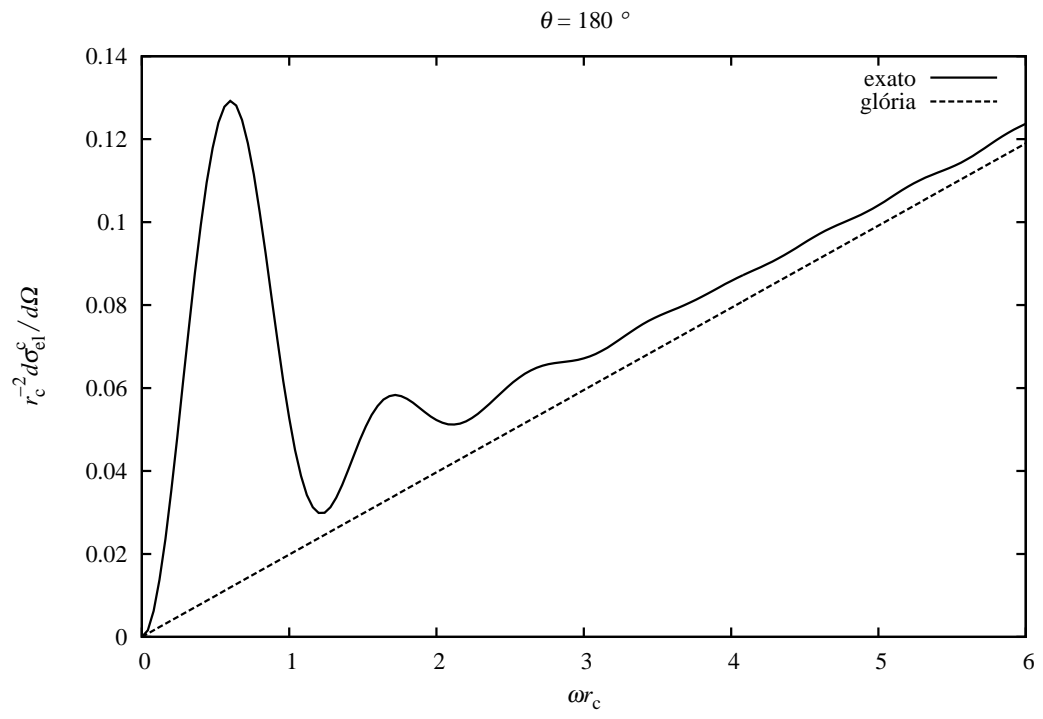

(b) Seção de choque diferencial de espalhamento de buracos acústicos canônicos no eixo para $\theta=180^{\circ}$. A linha tracejada representa a aproximação para o efeito glória.

Figura 5.13: Seção de choque de espalhamento de buracos acústicos canônicos no eixo de incidência como função da frequência. Observamos que para $\theta=0$, a seção de choque de espalhamento cresce muito rapidamente $\operatorname{com} \omega r_{\mathrm{c}}$. Para $\theta=180^{\circ}$, a intensidade de espalhamento apresenta algumas oscilações para pequenos valores $\omega r_{\mathrm{c}}$, mas depois cresce de forma aproximadamente linear com $\omega r_{\mathrm{c}}$, que é o resultado esperado da análise do efeito glória. 
mesmo valor da seção de choque de absorção para $\omega r_{\mathrm{c}} \rightarrow 0$ e cresce da mesma forma que a seção de choque de espalhamento.

A comparação entre a seção de choque diferencial de espalhamento do buraco negro de Schwarzschild e seu análogo acústico é mostrada na Figura 5.15 para $\omega r_{\mathrm{h}}=6.0$. Como devemos esperar, a seção de choque diferencial de espalhamento é bem maior para o caso de buracos negros de Schwarzschild. Isso é devido ao fato de que o espaço-tempo de buracos acústicos canônicos se torna plano muito mais rápido do que o espaço-tempo de buracos negros de Schwarzschild. Na mesma figura mostramos também a curva obtida com a fórmula do efeito glória para buracos acústicos canônicos, que é dada pela equação (4.68). Podemos ver que o resultado numérico está em excelente concordância com a aproximação do efeito glória para $\theta \rightarrow 180^{\circ}$.

\subsubsection{Caso eletromagnético em Schwarzschild}

Esta subseção trata do espalhamento do campo eletromagnético por buracos negros de Schwarzschild. Este resultado é bem recente e uma vez que ele foi obtido, a comparação entre a seção de choque diferencial de espalhamento de buracos negros de Schwarzschild para partículas não massivas de spins $0,1 / 2,1$ e 2 foi possível.

Iniciamos apresentando os resultados dessa subseção na Figura 5.16. Nela apresentamos a seção de choque diferencial eletromagnética de buracos negros de Schwarzschild para $M \omega=$ 2.0,3.0, 4.0. As linhas tracejadas da mesma figura são as aproximações para o efeito glória, (4.64). Podemos perceber que há uma excelente concordância do resultado numérico com a aproximação do efeito glória par $\theta>160^{\circ}$.

Na Figura 5.17 mostramos a comparação das seções de choque diferenciais de espalhamento de buracos negros de Schwarzschild para partículas não massivas de spins 0 [18, 61], $1 / 2$ [20], 1 [23] e 2 [15]. Podemos perceber que os resultados para campos bosônicos são bastante similares para $\theta<120^{\circ}$. Esta similaridade acontece já por volta de $\theta=160^{\circ}$ no caso dos campos eletromagnético e escalar não massivo. Já o resultado para o campo fermiônico oscila basicamente em oposição de fase com os casos bosônicos neste mesmo limite.

Com exceção do caso do campo escalar não massivo, todos as seções de choque diferenciais são zero quando $\theta=180^{\circ}$. Isto já foi previsto no contexto da aproximação para o efeito glória, analisado na seção 4.4. Este comportamento da seção de choque diferencial pode ser explicado, no caso do campo eletromagnético, com base no transporte paralelo do vetor de polarização ao longo do caminho descrito pelo raio espalhado. Consideremos um raio de luz incidente ao longo do eixo $z$ que orbita o buraco negro uma vez e retorna no sentido oposto $(\theta=\pi)$. Assumamos, sem perda de generalidade, que este raio possui um campo elétrico na direção do eixo $x$. Se o raio orbita no plano $x-z$, o campo será invertido, porém, se orbita no plano $y-z$, o campo se mantém. Portanto, pela integração sobre a degenerescência circular (todos os planos orbitais), existe um cancelamento perfeito. Este mesmo argumento pode ser usado para explicar os resultados nulos em $180^{\circ}$ para os campos de spin $1 / 2$ e 2 . 


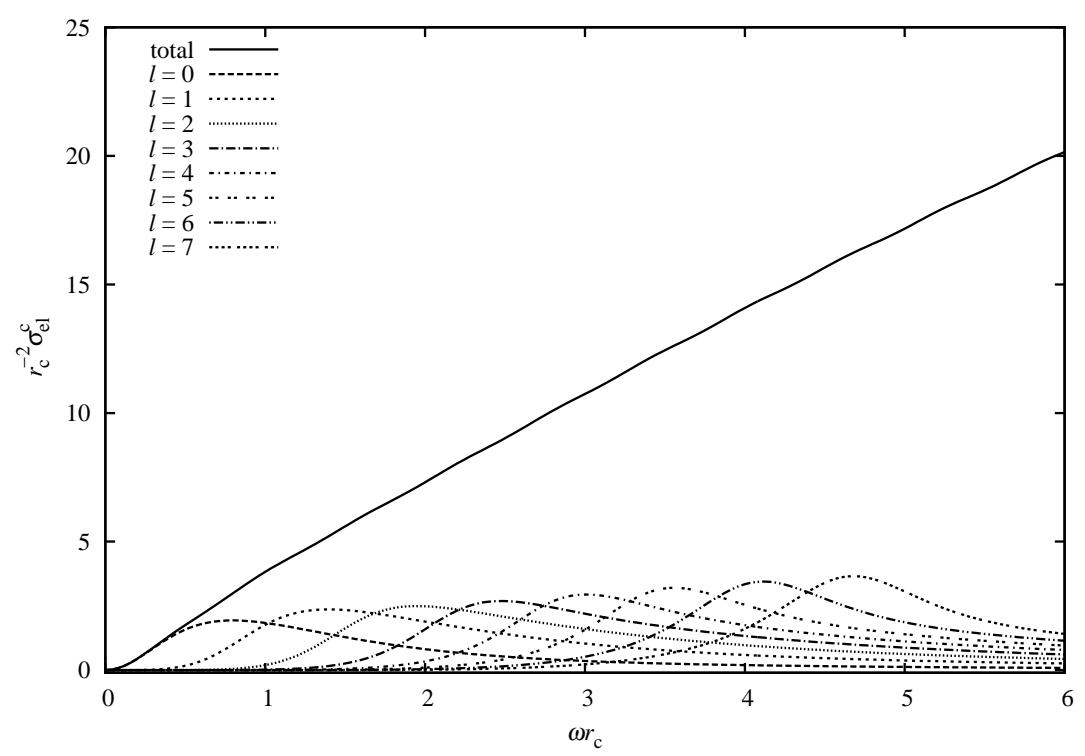

(a) Seção de choque de espalhamento de buracos acústicos canônicos. As contribuições parciais são mostradas até $l=7$.

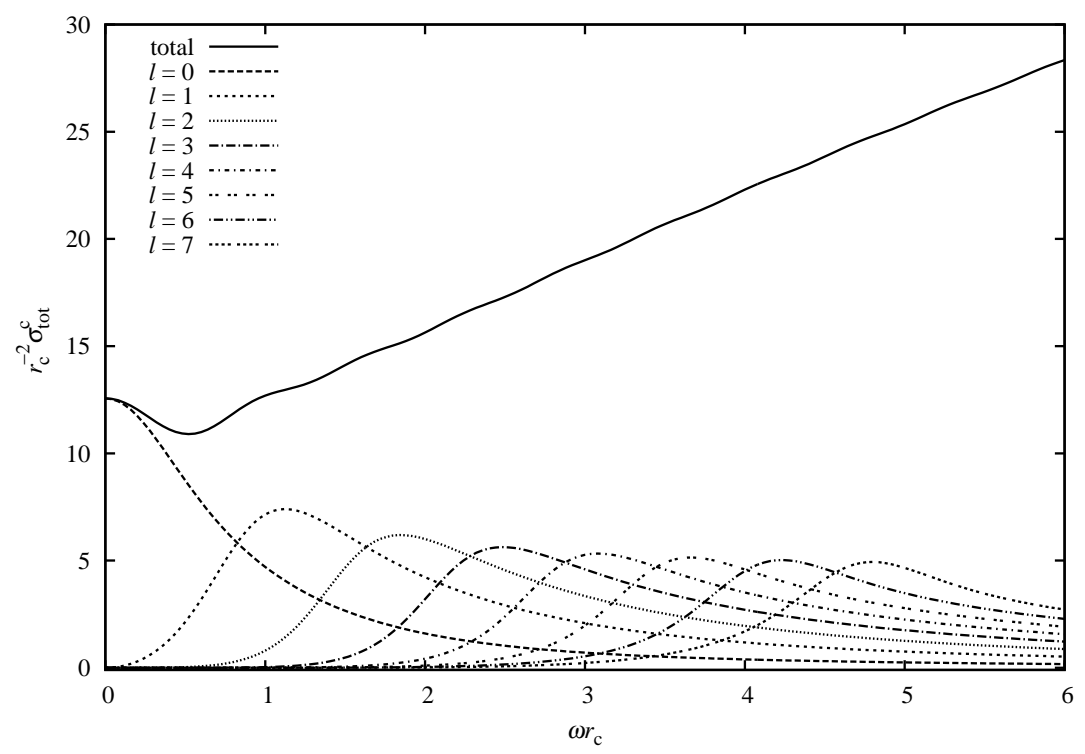

(b) Seção de choque total de buracos acústicos canônicos. As contribuições parciais são mostradas até $l=7$.

Figura 5.14: Diferentemente da seção de choque de absorção, a seção de choque de espalhamento cresce de forma aproximadamente linear com $\omega r_{c}$, (a), Este crescimento evidentemente se reflete na seção de choque total que é a soma das seções de choque de absorção e de espalhamento, (b). Uma vez que a seção de choque de espalhamento é zero para $\omega r_{\mathrm{c}} \rightarrow 0$, a seção de choque total assume o valor da seção de choque de absorção neste limite. 


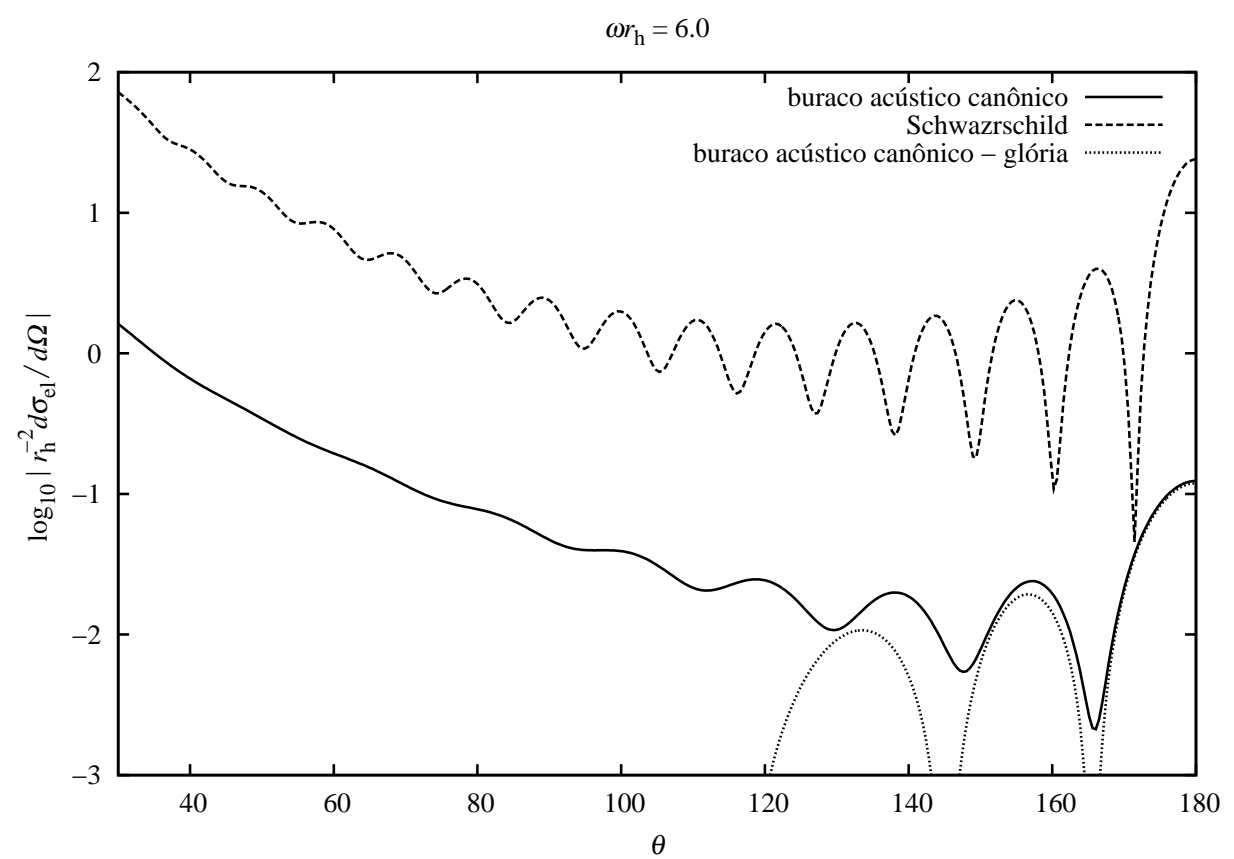

Figura 5.15: Comparação da seção de choque diferencial de espalhamento de buracos acústicos canônicos com o caso de buracos negros de Schwarzschild (escalar). Podemos ver que o resultado para buracos negros de Schwarzschild é bem maior (observe a escala logarítmica). O comprimento das franjas de interferência do espalhamento de buracos acústicos canônicos é maior. Aqui, a curva pontilhada representa o resultado para o efeito glória de buracos acústicos canônicos. Podemos perceber uma excelente concordância do cálculo numérico com a aproximação do efeito glória para $\theta>170^{\circ}$. 

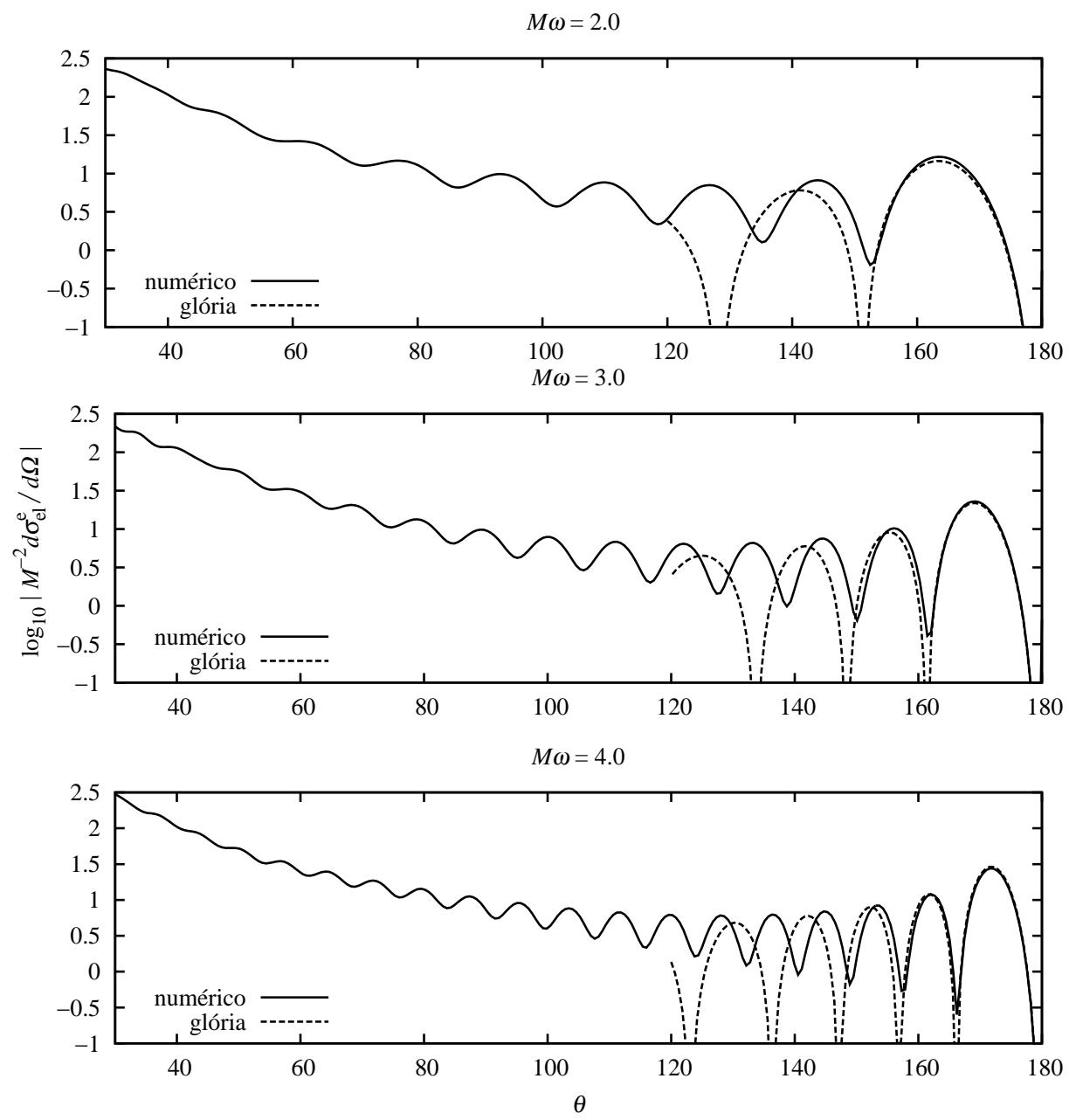

Figura 5.16: Seção de choque diferencial eletromagnética de buracos negros de Schwarzschild para $M \omega=2.0,3.0,4$.0 comparada com os resultados obtidos da aproximação para o efeito glória. 


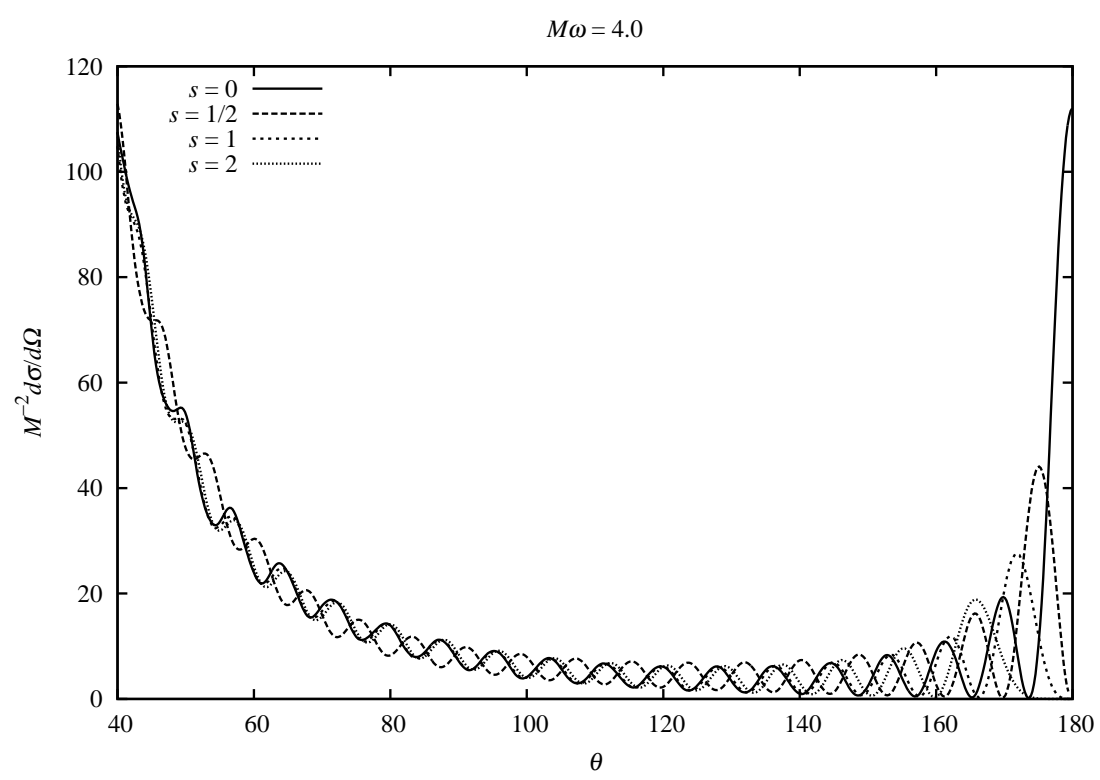

(a) Seção de choque diferencial de espalhamento de buracos negros de Schwarzschild para partículas não massivas de spin $0,1 / 2,1$ e 2 com $M \omega=4.0$.

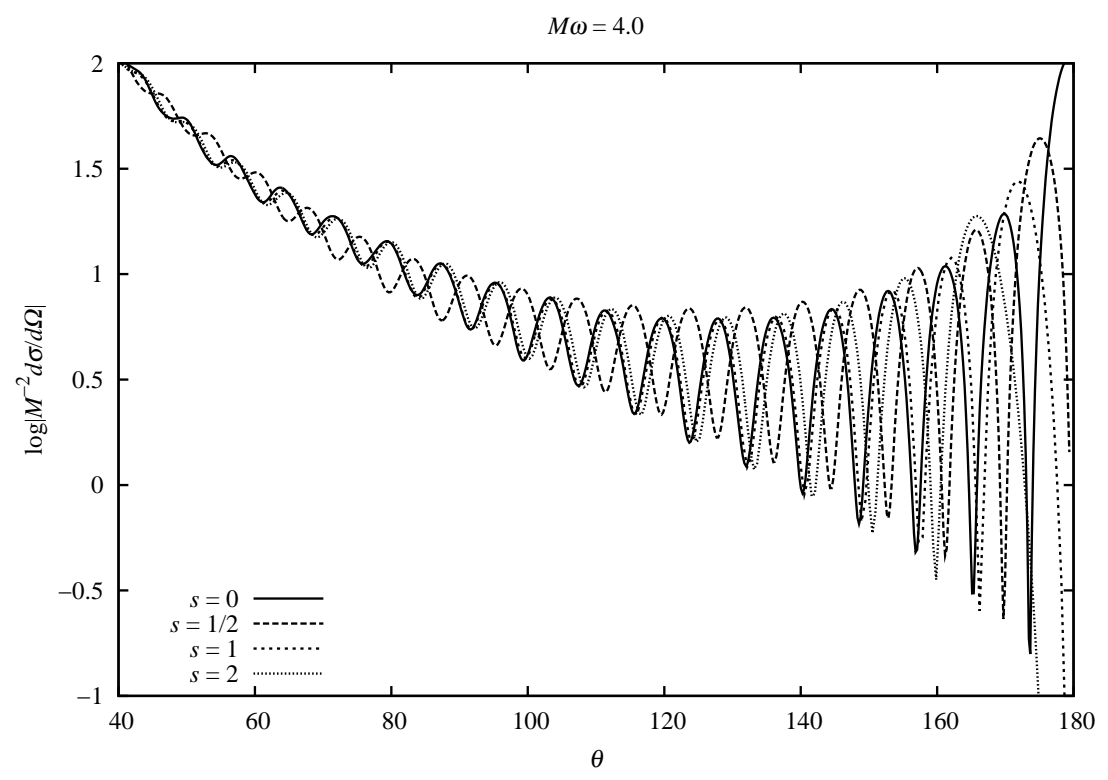

(b) $\mathrm{O}$ mesmo resultado plotado em (a) agora na escala logarítmica.

Figura 5.17: Seção de choque diferencial de espalhamento de buracos negros de Schwarzschild para $M \omega=4.0$. Com exceção de $\theta>160^{\circ}$, os resultados para os campos de spins 0 e 1 oscilam de forma muito semelhante. O resultado do campo de spin 2 acompanha melhor as oscilações dos resultados para os campos de spins 0 e 1 para $\theta<120^{\circ}$, enquanto que o resultado para 0 campo de spin 1/2 oscila basicamente em oposição de fase aos resultados dos casos bosônicos neste mesmo limite. 


\section{Capítulo 6}

\section{Conclusão}

Aqui mostramos o estudo da absorção e do espalhamento de buracos negros de Schwarzschild para ondas eletromagnéticas e de análogos acústicos destes buracos negros. Apresentamos também a absorção e o espalhamento do campo escalar não massivo por buracos negros de Reissner-Nordström. Os principais resultados apresentados aqui foram obtidos de forma numérica. Isto se deve ao fato de as soluções da parte radial das equações de campo não poderem ser escritas em temos de funções conhecidas.

No caso de buracos negros de Schwarzschild, tanto para o campo de spin 0 quanto de spin 1, e de Reissner-Nordström, aplicamos um método de convergência para obter a seção de choque diferencial de espalhamento de forma precisa. No caso do buraco acústico canônico, defasagens para $l \gg \omega r_{\mathrm{c}}$ foram encontradas analiticamente, de modo que obtivemos a seção de choque diferencial de espalhamento destes objetos com excelente precisão, sem a necessidade de utilizar métodos de convergência.

Aproximações analíticas foram apresentadas para a seção de choque de absorção em baixas e altas frequências e para a seção de choque diferencial de espalhamento para pequenos e grandes ângulos. A análise do comportamento de geodésicas ao redor dos espaços-tempos em questão foi de fundamental importância. Por meio desta análise foi possível obter a seção de choque de absorção em altas frequências e os parâmetros necessários à abordagem do efeito glória. Esta análise nos permitiu perceber também que as principais características das seções de choque, nos limites de altas frequências e grande ângulos, estão diretamente relacionadas à órbita instável de partículas não massivas.

Vimos que a seção de choque de absorção de buracos negros de Reissner-Nordström diminui quando o razão entre a intensidade de sua carga e massa, $q$, cresce (Figura 5.3). Isto está de acordo com o fato de que os raios da órbita instável de partículas não massivas e do horizonte de eventos do buraco negro diminuem quando $q$ aumenta. Também verificamos que quando a carga elétrica do buraco negro aumenta, as larguras das franjas de interferência da seção de choque diferencial de espalhamento aumentam (Figura 5.9). Vimos também que não há uma variação monotônica da magnitude da seção de choque diferencial de espalhamento como há na seção de choque de absorção (Figuras 5.9 e 5.10), para buracos negros carregados. De acordo com o estudo do efeito glória, isto se deve ao fato de que, apesar de o parâmetro de 
impacto referente a ângulos de espalhamento $\theta=\pi, b_{\mathrm{g}}$, diminuir [Figura 5.11(a)], a quantidade $|d b / d \theta|_{\theta=\pi}$ aumenta [Figura 5.11(b)].

As seções de choque de buracos acústicos canônicos são em geral menores do que as obtidas no caso de buracos negros de Schwarzschild. Isto é consequência do fato de que a métrica destes objetos se torna plana com $r^{-4}$, bem mais rápido que a métrica de Schwarzschild, que se torna plana com $r^{-1}$. A seção de choque de absorção tende à área do horizonte de eventos no limite $\omega r_{\mathrm{h}} \rightarrow 0$, independente da forma do espaço-tempo [56, 57] (Figura 5.5). Em números, a magnitude do efeito glória de buracos acústicos canônicos é cerca de 170 vezes menor que o valor equivalente no caso de buracos negros de Schwarzschild quando fixamos $\omega r_{\mathrm{h}}$ [65]. Já a largura das franjas de interferência para buracos acústicos canônicos é cerca de 1.66 maior que no caso de buracos negros de Schwarzschild (Figura 5.15).

Algo particularmente interessante no espalhamento de buracos acústicos canônicos é que a seção de choque diferencial de espalhamento é finita para todos os valores de $\theta$. Portanto, a seção de choque de espalhamento e a seção de choque total são finitas para valores finitos de $\omega r_{\mathrm{c}}$ [Figuras 5.14(a) e 5.14(b)]. Este comportamento é diferente quando analisamos o espalhamento por buracos negros de Schwarzschild, onde a seção de choque diferencial de espalhamento diverge quando $\theta \rightarrow 0$ [18, 60, 61]. Isto pode ser explicado quando tomamos o limite de campo fraco. Nesse caso, a função radial da equação de Klein-Gordon obedece uma equação tipo Schrödinger na qual o potencial efetivo de buracos negros de Schwarzschild é proporcional à $r^{-1}$ [equação 4.52]]. No mesmo limite, a maior contribuição para o potencial efetivo de buracos acústicos canônicos é proporcional a $r^{-4}$ [equações [5.4] e [5.5]].

Vimos que a seção de choque diferencial de espalhamento eletromagnética de buracos negros de Schwarzschild vai a zero quando $\theta \rightarrow 180^{\circ}$. Isto é explicado pela interferência destrutiva total causada devido ao transporte paralelo do vetor de polarização do campo eletromagnético [15, 37]. O mesmo ocorre ainda para partículas fermiônicas e para ondas gravitacionais e pode ser explicado de modo análogo ao caso eletromagnético. Comparamos a seção de choque diferencial de espalhamento de buracos negros de Schwarzschild para partículas de spins 0, 1/2, 1 e 2 (Figura 5.17). Através desta comparação, concluímos que os resultados para campos bosônicos são bastante similares quando $\theta<120^{\circ}$. Neste mesmo limite, o resultado para o campo fermiônico oscila basicamente em oposição de fase aos resultados bosônicos.

Os resultados acima se juntam a um grande conjunto de pesquisas realizadas na área de espalhamento e absorção por buracos negros, sendo que a maioria destas pesquisas se concentra nos casos de buracos negros de Schwarzschild e Kerr. Como vimos aqui, o estudo do espalhamento por buracos negros carregados é bastante interessante, podendo, em princípio, ser utilizado como ferramenta para detecção da carga destes objetos.

A possibilidade da observação da radiação Hawking em laboratório com os análogos gravitacionais de buracos negros trouxe mais uma área de aplicação também para a teoria de espalhamento. Nesse sentido, o estudo de espalhamento e absorção por buracos acústicos é um dos passos que pode trazer os fenômenos que ocorrem ao redor de buracos negros para mais próximo da verificação experimental [24, 36, 63]. 


\section{Referências Bibliográficas}

[1] R. M. Wald, General Relativity (The University of Chicago Press, Chicago, 1984).

[2] M. P. Hobson, G. P. Efstathiou, e A. N. Lasenby, General Relativity (Cambridge University Press, 2006).

[3] C. M. Will, Living Rev. Relativity 9, 3 (2006), http://www.livingreviews.org//rr-2006-3.

[4] M. C. Begelman, Science 300, 1898 (2003).

[5] S. Chandrasekhar, The Mathematical Theory of Black Holes (Oxford University Press, New York, 1983).

[6] S. W. Hawking, Nature 248, 30 (1974).

[7] A. A. Starobinsky, Zh. Exp. i Teoret. Fiz. 64, 48 (tradução no Soviet Phys. JETP 37, 28).

[8] U. H. Gerlach, Phys. Rev. Lett. 32, 1023 (1974).

[9] F. J. Zerilli, Phys. Rev. D 9, 860 (1974).

[10] V. Moncrief, Phys. Rev. D 9, 2707 (1974); 10, 1057 (1974); 12, 1526 (1975).

[11] D. W. Olson e W. G. Unruh, Phys. Rev. Lett. 33, 1116 (1974).

[12] R. A. Matzner, Phys. Rev. D 14, 3274 (1976).

[13] L. C. B. Crispino e E. S. Oliveira, Phys. Rev. D 78, 024011 (2008).

[14] L. C. B. Crispino, A. Higuchi e E. S. Oliveira, Phys. Rev. D 80, 104026 (2009).

[15] J. A. H. Futterman, F. A. Handler e R. A. Matzner, Scattering from Black Holes (Cambridge University Press, Cambridge, England, 1988).

[16] D. N. Page, Phys. Rev. D 13, 198 (1976).

[17] N. Sanchez, Phys. Rev. D 18, 1030 (1978).

[18] N. Sanchez, Phys. Rev. D 18, 1798 (1978). 
[19] C. Doran, A. Lasenby, S. Dolan e I. Hinder, Phys. Rev. D 71, 124020 (2005).

[20] S. Dolan, C. Doran e A. Lasenby, Phys. Rev. D 74, 064005 (2006).

[21] L. C. B. Crispino, E. S. Oliveira, A. Higuchi e G. E. A. Matsas, Phys. Rev. D 75, 104012 (2007).

[22] S. R. Dolan, Class. Quantum Grav. 25, 235002 (2008).

[23] L. C. B. Crispino, S. R. Dolan e E. S. Oliveira, Phys. Rev. Lett. 102, 231103 (2009).

[24] W. G. Unruh, Phys. Rev. Lett. 46, 1351 (1981).

[25] R. Schützhold e W. G. Unruh, Phys. Rev. Lett. 95, 031301 (2005).

[26] S.-W. Kim, W. T. Kimb e J. J. Oh, Phys. Lett. B 608, 10 (2005).

[27] C. Barceló, S. Liberati, S. Sonego e M. Visser, Phys. Rev. Lett. 97, 171301 (2006).

[28] S. Basak e P. Majumdar, Class. Quantum Grav. 20, 2929 (2003).

[29] T. R. Slatyer e C. M. Savage, Class. Quantum Grav. 22, 3833 (2005).

[30] C. Cherubini, F. Federici, S. Succi e M. P. Tosi, Phys. Rev. D 72, 084016 (2005).

[31] K. Choy, T. Kruk, M. E. Carrington, T. Fugleberg, J. Zahn, R. Kobes, G. Kunstatter e D. Pickering, Phys. Rev. D 73, 104011 (2006).

[32] E. Berti, V. Cardoso e J. P. S. Lemos, Phys. Rev. D 70, 124006 (2004).

[33] R. Balbinot, S. Fagnocchi, A. Fabbri e G. P. Procopio, Phys. Rev. Lett. 94, 161302 (2005).

[34] R. Balbinot, S. Fagnocchi e A. Fabbri, Phys. Rev. D 71, 064019 (2005).

[35] C. Barceló, S. Liberati, e M. Visser, Int. J. Mod. Phys. D 12, 1641 (2003).

[36] M. Visser, Class. Quantum Grav. 15, 1767 (1998).

[37] T.-R. Zhang e C. DeWitt-Morette, Phys. Rev. Lett. 52, 2313 (1984).

[38] R. A. d'Inverno, Introducing Einstein's Relativity (Clarendon Press, Oxford, 1992).

[39] G. E. A. Matsas e A. R. R. Silva, Phys. Rev. Lett. 99, 181301 (2007).

[40] L. D. Landau e E. M. Lifshitz, Mechanics (Butterworth-Heinenann, Oxford, 1981), 3a. edição.

[41] L. C. B. Crispino, S. R. Dolan e E. S. Oliveira, Phys. Rev. D 79064022 (2009). 
[42] B. Mashhoon, Phys. Rev. D 7, 2807 (1973).

[43] M. Abramowitz e I. A. Stegun, Handbook of Mathematical Functions (Dover Publications, New York, 1965).

[44] W. G. Unruh, Phys. Rev. D 51, 2827 (1995).

[45] K. Gottfried e T.-M. Yan, Quantum Mechanics: Fundamentals (Springer, New York, 2004), 2a. edição.

[46] G. Arfken, Mathematical Methods for Physicists (Academic Press, California, 1985), 3a. edição.

[47] L. C. B. Crispino, A. Higuchi e G. E. A. Matsas, Phys. Rev. D 63, 124008 (2001).

[48] L. C. B. Crispino, Quantização a baixas freqüências de campos bosônicos no espaçotempo de Schwarzschild e aplicações, Tese de Doutorado, IFT-UNESP (2001).

[49] A. Higuchi, Class. Quantum Grav. 4, 721 (1987).

[50] C. C.-Tannoudji, B. Diu e F. Laloë, Quantum Mechanics, volume II (John Wiley \& Sons, New York, 1977).

[51] E. F. Eiroa, G. E. Romero e D. F. Torres, Phys. Rev. D 66, 024010 (2002).

[52] A. Bhadra, Phys. Rev. D 67, 103009 (2003).

[53] M. Sereno, Phys. Rev. D 69, 023002 (2004).

[54] R. Fabbri, Phys. Rev. D 12, 933 (1975).

[55] H. C. van de Hulst, Ligth Scattering by Small Particles (Wiley, New York, 1957).

[56] S. R. Das, G. Gibbons e S. D. Mathur, Phys. Rev. Lett. 78, 417 (1997).

[57] A. Higuchi, Class. Quantum Grav. 18, L139 (2001); 19, 599 (2002).

[58] P. J. Westervelt, Phys. Rev. D 3, 2319 (1971).

[59] P. C. Peters, Phys. Rev. D 13, 775 (1976).

[60] N. G. Sanchez, J. Math. Phys. 17, 688 (1976).

[61] N. Andersson, Phys. Rev. D 52, 1808 (1995).

[62] R. A. Matzner, C. DeWitt-Morette, B. Nelson e T.-R. Zhang, Phys. Rev. D 31, 1869 (1985).

[63] S. R. Dolan, E. S. Oliveira e L. C. B. Crispino, Phys. Rev. D 79, 064014 (2009). 
[64] C. Darwin, Proc. R. Soc. A 249, 180 (1959).

[65] Note que as grandezas não são exatamente as mesmas. Quando comparamos buracos acústicos canônicos com buracos negros de Schwarzschild, estamos comparando razões das seções de choque com os tamanhos dos buracos, basicamente com o raio do horizonte $r_{\mathrm{h}}$. Além disso, as variáveis adimensionais $\omega r_{\mathrm{h}}$ são diferentes. Quando restituímos as velocidades da luz, $c$, e do som no fluido, $c_{\mathrm{som}}$, devemos ter $\omega_{\mathrm{som}} r_{\mathrm{c}} / c_{\mathrm{som}}=\omega_{\mathrm{ce}} r_{\mathrm{S}} / c$, na qual "ce" abrevia campo escalar não massivo. Então, se tivermos buracos com raios de mesmo tamanho $\left(r_{\mathrm{c}}=r_{\mathrm{S}}\right), \omega_{\mathrm{som}} \neq \omega_{\mathrm{ce}}$, porém $\omega_{\mathrm{som}} / c_{\mathrm{som}}=\omega_{\mathrm{ce}} / c$.

[66] D. R. Yennie, D. G. Ravenhall e R. N. Wilson, Phys. Rev. 95, 500 (1954).

[67] K.W. Ford e J. A. Wheeler, Ann. Phys. (N.Y.) 7, 259 (1959).

[68] I. S. Gradshteyn e I. M. Ryzhik, Tables of Integrals, Series, and Products (Academic Press, New York, 1980).

[69] E. Jung e D. K. Park, Nucl.Phys. B 717, 272 (2005).

[70] L. C. B. Crispino, G. E. A. Matsas e E. S. Oliveira, Phys. Rev. D 76, 107502 (2007). 